\title{
Análise dos resultados de ensaios de proficiência via modelos de regressão com variável explicativa aleatória ${ }^{1}$
}

\author{
Aline Othon Montanari
}

Orientador: Prof. Dr. Dorival Leão Pinto Jr.

Dissertação apresentada ao Instituto de Ciências Matemáticas e de Computação - ICMC-USP, como parte dos requisitos para obtenção do título de Mestre em Ciências de Computação e Matemática Computacional.

USP - São Carlos

Maio/2004 
Análise dos resultados de ensaios de proficiência via modelos de regressão com variável explicativa aleatória

Aline Othon Montanari 
À minha mãe, Célia, por ensinar-me a não desistir dos meus sonhos e ao meu pai, Rogério, pelo exemplo de caráter e por confiar na minha competência. 



\section{Agradecimentos}

Agradeço a Deus pelo dom que me foi concedido e por me acompanhar em todos os dias de minha vida.

Agradeço, em especial, aos meus pais Rogério e Célia, por incentivarem meus estudos e por sempre lutarem e em alguns momentos se sacrificarem para que eu tivesse a melhor formação profissional possível e a minha irmã, Laura, pela amizade. Agradeço a todos os meus parentes, em especial meu tio Marcelo, que torceram para que eu conquistasse mais esta etapa.

Agradeço, em particular, ao meu namorado Fábio, pelo amor, carinho e compreensão.

Um agradecimento especial para o meu amigo Daniel, pelo apoio e por todo tempo que me ajudou na realização deste trabalho.

Agradeço ao meu orientador Prof. Dr. Dorival Leão Pinto Junior pela orientação, pelas aulas e pelos seus ensinamentos, que foram muito importantes para o término desta dissertação.

Também quero dizer que me sinto muito feliz de ter encontrado durante o período da graduação grandes amigos, como o Alex, a Mariana, a Grazielle, a Kelly e a Luci. Em especial meus grandes companheiros, aliás, verdadeiros irmãos Flávia e Mário (Ronaldinho).

Agradeço os meus colegas do mestrado, Gecirlei, Fabrízio, Paulo, Ricardo e Juliana, pelas contribuições, por cada dia de estudo e pelos momentos de descontração.

Agradeço também os meus amigos de Araraquara: Renata, Sabrina, Ana Paula, Christiano e Vitor que não se esqueceram de 10 anos de amizade e companheirismo.

Aos professores da estatística Marinho, Reiko e Jorge pelos ensinamentos e dedicação, a minha orientadora de graduação Prof . Sandra Godoy por ser a primeira a me ensinar como ser uma pesquisadora e aos funcionários do Instituto de Ciências Matemáticas e de Computação pela gentileza e atenção diariamente prestadas.

Um obrigado especial a minha tia Géria por compartilhar comigo os meus momentos de alegria e tristeza e por ter me acolhido por 6 anos em sua casa neste período de estudos.

Agradeço a Capes pelo auxílio financeiro.

Enfim, a todos aqueles que colaboraram de alguma forma para a realização deste trabalho.

Muito Obrigada!

"Há homens que lutam um dia e são bons. Há outros que lutam um ano e são melhores. Há os que lutam muitos anos e são muito bons. Mas há os que lutam toda a vida, e estes são imprescindíveis" (Bertold Brecht). 



\section{Resumo}

Em um programa de ensaio de proficiência (EP) conduzido pelo Grupo de Motores, um grupo de onze laboratórios da área de temperatura realizaram medições em cinco pontos da escala de um termopar.

Neste trabalho, propomos um modelo de regressão com variável explicativa X (aleatória) representando o termopar padrão que denominaremos por artefato e a variável dependente Y representando as medições dos laboratórios. O procedimento para a realização da comparação é simples, ambos termopares são colocados no forno e as diferenças entre as medições são registradas. Para a análise dos dados, vamos trabalhar com a diferença entre a diferença das medições do equipamento do laboratório e o artefato, e o valor de referência (que é determinado por 2 laboratórios que pertencem a Rede Brasileira de Calibração (RBC)).

O erro de medição tem variância determinada por calibração, isto é, conhecida. Assim, vamos encontrar aproximações para as estimativas de máxima verossimilhança para os parâmetros do modelo via algoritmo EM. Além disso, propomos uma estratégia para avaliar a consistência dos laboratórios participantes do programa de EP. 



\section{Abstract}

In a program of proficiency assay, a group of eleven laboratories of the temperature area had carried through measurements in five points on the scale of the thermopair.

In this work, we propose a regression model with a random explanatory variable representing the temperature measured by the standard thermopair, which will be called device. The procedure for the comparison accomplishment is as follows. The device and the laboratory's thermopair to be tested are placed in the oven and the differences between the measurements are registered. For the analysis of the data, the response variable is the difference between those difference and the reference value, which is determined by two laboratories that belong to the Brazilian Net of Calibration (RBC).

The measurement error has variance determined by calibration which is known. Therefore, we find the maximum likelihood estimates for the parameters of the model via EM algorithm. We consider a strategy to establish the consistency of the participant laboratories of the program of proficiency assay. 



\section{Sumário}

1 Introdução 1

1.1 Objetivo . . . . . . . . . . . . . . . . . . . . 2

1.2 Organização do Trabalho . . . . . . . . . . . . . . . . . . 2

2 Apresentação dos Dados e Avaliação das Incertezas de Medição 3

2.1 Introdução . . . . . . . . . . . . . . . . . . . . . . . . . 3

2.2 Apresentação dos dados . . . . . . . . . . . . . . . . . . . . . . . 3

2.3 Avaliação da Incerteza de Medição . . . . . . . . . . . . . . . . . . . . . 16

2.3.1 Equação de Medição . . . . . . . . . . . . . . . . . . . . . . . . . . 17

2.3.2 Avaliação da Incerteza Padrão de Medição das Estimativas de Entrada 18

2.3.3 Avaliação da Incerteza Padrão Combinada . . . . . . . . . . . . . . . 20

2.3.4 Incerteza Expandida de Medição . . . . . . . . . . . . . . . . . . . 21

3 Modelo Estatístico, Estimação e Algoritmo EM 25

3.1 Introdução . . . . . . . . . . . . . . . . . . . . . . . . . 25

3.2 Modelo Proposto . . . . . . . . . . . . . . . . . . . . . . . 25

3.3 Introdução da Variável Latente e Algoritmo EM . . . . . . . . . . . . . . . . 27

3.3 .1 Etapa E . . . . . . . . . . . . . . . . . . . . . 31

3.3 .2 Etapa M . . . . . . . . . . . . . . . . . . . 31

3.3 .3 Algoritmo EM . . . . . . . . . . . . . . . . . . . 33

3.4 Estudo da Convergência do Algoritmo EM . . . . . . . . . . . . . . . . . 33

3.4.1 Formulação do Algoritmo EM . . . . . . . . . . . . . . . . . . . . 33

3.4 .2 Passo M Aplicado ao Nosso Modelo . . . . . . . . . . . . . . . . . . . 34

3.4 .3 Convergência do Algoritmo EM . . . . . . . . . . . . . . . . 35

4 Aplicações $\quad 37$

4.1 Introdução . . . . . . . . . . . . . . . . . . . . . . . 37

4.2 Estimativas para os Parâmetros via Algoritmo EM . . . . . . . . . . . . . . 37

4.3 Análise da Competência dos Laboratórios . . . . . . . . . . . . . . . . . . . 39

5 Considerações Finais $\quad 49$

5.1 Conclusões . . . . . . . . . . . . . . . . . . . . . . . . . 49

5.2 Propostas de Trabalhos Futuros . . . . . . . . . . . . . . . . . . . . 49

$\begin{array}{ll}\text { A Dados dos Laboratórios } & 51\end{array}$

B Tabelas de Incertezas dos Laboratórios $\quad 55$

$\begin{array}{ll}\text { C Algoritmo EM } & 71\end{array}$

D Função Geradora do Vetor de Médias $\quad 83$ 
E Obtenção do Intervalo de Confiança $\quad 85$

Referências Bibliográficas $\quad 89$ 


\section{Lista de Figuras}

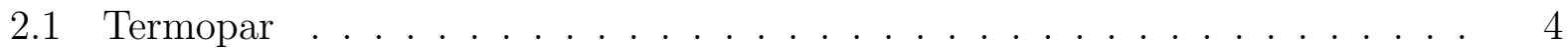

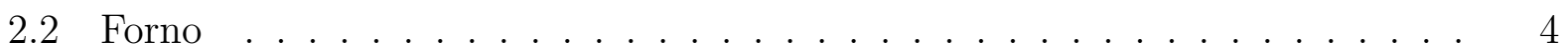

2.3 Ensaio . . . . . . . . . . . . . . . . . . . . . . . 4

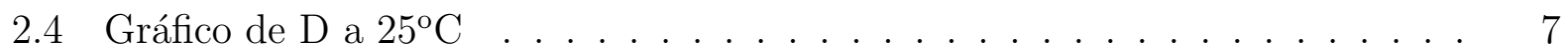

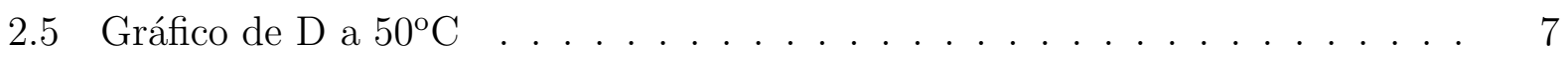

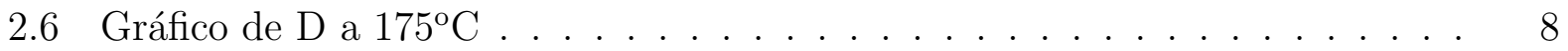

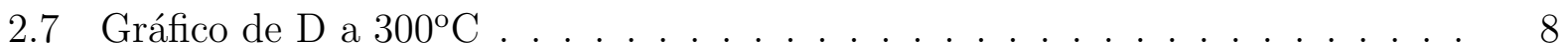

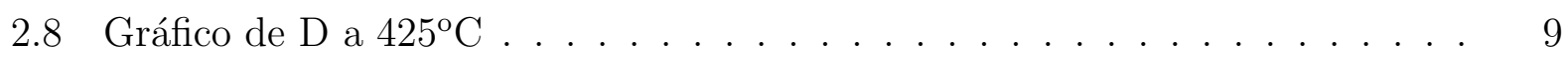

2.9 Gráfico da tendência do laboratório $1 \ldots \ldots$. . . . . . . . . . . . 10

2.10 Gráfico da tendência do laboratório $2 \ldots \ldots \ldots$. . . . . . . . . 11

2.11 Gráfico da tendência do laboratório $3 \ldots \ldots$. . . . . . . . . . 11

2.12 Gráfico da tendência do laboratório 4 . . . . . . . . . . . . . . . . . 12

2.13 Gráfico da tendência do laboratório $5 \ldots \ldots \ldots \ldots$

2.14 Gráfico da tendência do laboratório $6 \ldots \ldots \ldots \ldots$

2.15 Gráfico da tendência do laboratório $7 \ldots \ldots \ldots \ldots$

2.16 Gráfico da tendência do laboratório $8 \ldots \ldots$. . . . . . . . . . . . . 14

2.17 Gráfico da tendência do laboratório $9 \ldots \ldots \ldots \ldots \ldots$

2.18 Gráfico da tendência do laboratório $10 \ldots \ldots \ldots \ldots$

2.19 Gráfico da tendência do laboratório $11 \ldots \ldots$. . . . . . . . . . . 15

4.1 Tendência, Reta Ajustada e Intervalo de Confiança para o laboratório 1 . . . 40

4.2 Tendência, Reta Ajustada e Intervalo de Confiança para o laboratório 2 . . . 41

4.3 Tendência, Reta Ajustada e Intervalo de Confiança para o laboratório 3 . . . 41

4.4 Tendência, Reta Ajustada e Intervalo de Confiança para o laboratório 4 . . . 42

4.5 Tendência, Reta Ajustada e Intervalo de Confiança para o laboratório 5 . . . 43

4.6 Tendência, Reta Ajustada e Intervalo de Confiança para o laboratório 6 . . . 43

4.7 Tendência, Reta Ajustada e Intervalo de Confiança para o laboratório 7 . . . 44

4.8 Tendência, Reta Ajustada e Intervalo de Confiança para o laboratório 8 . . . 45

4.9 Tendência, Reta Ajustada e Intervalo de Confiança para o laboratório 9 . . . 45

4.10 Tendência, Reta Ajustada e Intervalo de Confiança para o laboratório 10 . . 46

4.11 Tendência, Reta Ajustada e Intervalo de Confiança para o laboratório 11 . . 47 


\section{Lista de Tabelas}

2.1 Dados dos laboratórios de referência Salcas e Consistec $\left(\mathrm{em}^{\circ} \mathrm{C}\right) \ldots \ldots$

2.2 Roteiro seguido pelo artefato . . . . . . . . . . . . . . . . 5

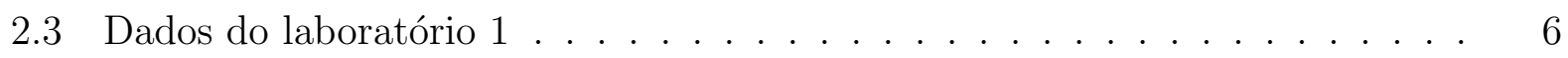

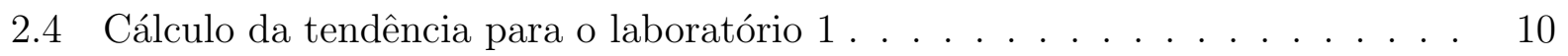

2.5 Cálculo de incertezas para o laboratório 1 a $25^{\circ} \mathrm{C} \ldots \ldots . \ldots 23$

4.1 Teste 1 - Valores das Estimativas para os Parâmetros via Algoritmo EM . 37

4.2 Teste 2 - Valores das Estimativas para os Parâmetros via Algoritmo EM . . 38

4.3 Teste 3 - Valores das Estimativas para os Parâmetros via Algoritmo EM . . 39

4.4 Intervalos de $95 \%$ de confiança para a tendência $\left({ }^{\circ} C\right)$ - Laboratório $1 \ldots 40$

4.5 Intervalos de $95 \%$ de confiança para a tendência $\left({ }^{\circ} \mathrm{C}\right)$ - Laboratório $2 \ldots 40$

4.6 Intervalos de $95 \%$ de confiança para a tendência $\left({ }^{\circ} C\right)$ - Laboratório $3 \ldots$. . . 41

4.7 Intervalos de $95 \%$ de confiança para a tendência $\left({ }^{\circ} C\right)$ - Laboratório $4 \ldots 42$

4.8 Intervalos de $95 \%$ de confiança para a tendência $\left({ }^{\circ} C\right)$ - Laboratório 5 . . . . 42

4.9 Intervalos de $95 \%$ de confiança para a tendência $\left({ }^{\circ} C\right)$ - Laboratório $6 \ldots 43$

4.10 Intervalos de $95 \%$ de confiança para a tendência $\left({ }^{\circ} C\right)$ - Laboratório 7 . . . 44

4.11 Intervalos de $95 \%$ de confiança para a tendência $\left({ }^{\circ} C\right)$ - Laboratório $8 \ldots$. . . 44

4.12 Intervalos de $95 \%$ de confiança para a tendência $\left({ }^{\circ} C\right)$ - Laboratório $9 \ldots 45$

4.13 Intervalos de $95 \%$ de confiança para a tendência $\left({ }^{\circ} C\right)$ - Laboratório $10 \ldots$. . 46

4.14 Intervalos de $95 \%$ de confiança para a tendência $\left({ }^{\circ} C\right)$ - Laboratório 11 . . . 46

A.1 Dados do laboratório $2 \ldots \ldots \ldots \ldots$. . . . . . . . . . . . . . . . 51

A.2 Dados do laboratório $3 \ldots \ldots \ldots \ldots$. . . . . . . . . . . . . . . 51

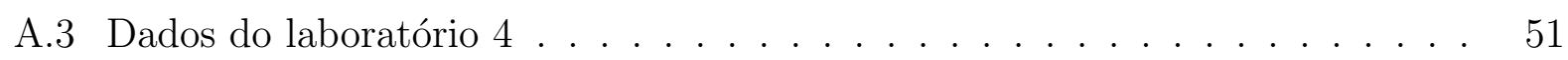

A.4 Dados do laboratório $5 \ldots \ldots \ldots \ldots$. . . . . . . . . . . . . . . . 52

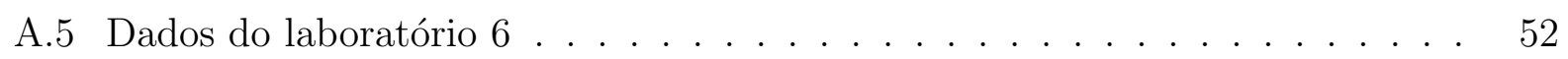

A.6 Dados do laboratório $7 \ldots \ldots \ldots \ldots$. . . . . . . . . . . . . . 52

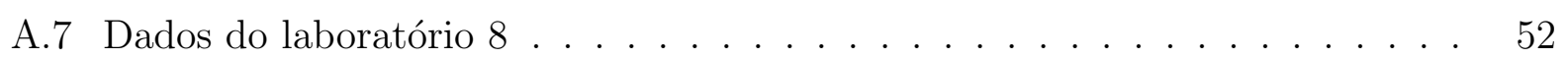

A.8 Dados do laboratório $9 \ldots \ldots \ldots \ldots \ldots$

A.9 Dados do laboratório $10 \ldots \ldots \ldots \ldots \ldots$

A.10 Dados do laboratório $11 \ldots \ldots \ldots \ldots$

B.1 Cálculo de incertezas para o laboratório 1 a $50^{\circ} \mathrm{C} \ldots \ldots$. . . . . . . . 55

B.2 Cálculo de incertezas para o laboratório 1 a $175^{\circ} \mathrm{C} \ldots \ldots \ldots 5$

B.3 Cálculo de incertezas para o laboratório 1 a $300^{\circ} \mathrm{C} \ldots \ldots \ldots$. . . . . . . . . . . . . . . 56

B.4 Cálculo de incertezas para o laboratório 1 a $425^{\circ} \mathrm{C} \ldots \ldots \ldots$. . . . . . . 56

B.5 Cálculo de incertezas para o laboratório 2 a $25^{\circ} \mathrm{C} \ldots \ldots \ldots$

B.6 Cálculo de incertezas para o laboratório 2 a $50^{\circ} \mathrm{C} \ldots \ldots \ldots$

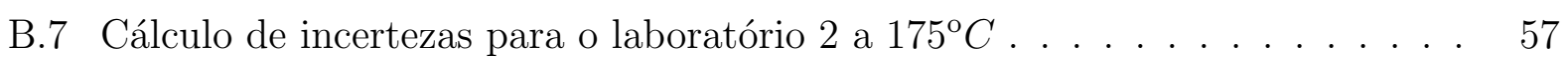

B.8 Cálculo de incertezas para o laboratório 2 a $300^{\circ} \mathrm{C} \ldots \ldots$. . . . . . . . . 57 
B.9 Cálculo de incertezas para o laboratório 2 a $425^{\circ} \mathrm{C} \ldots \ldots$. . . . . . . 57

B.10 Cálculo de incertezas para o laboratório 3 a $25^{\circ} \mathrm{C} \ldots \ldots$. . . . . . . . . 57

B.11 Cálculo de incertezas para o laboratório 3 a $50^{\circ} \mathrm{C} \ldots \ldots$. . . . . . . . . . . . . . . . . . . . 58

B.12 Cálculo de incertezas para o laboratório 3 a $175^{\circ} \mathrm{C} \ldots \ldots$. . . . . . . . 58

B.13 Cálculo de incertezas para o laboratório 3 a $300^{\circ} \mathrm{C} \ldots \ldots$. . . . . . . . . . . . . . . . . . 58

B.14 Cálculo de incertezas para o laboratório 3 a $425^{\circ} \mathrm{C} \ldots \ldots$. . . . . . . . . 58

B.15 Cálculo de incertezas para o laboratório 4 a $25^{\circ} \mathrm{C} \ldots \ldots$. . . . . . . . 59

B.16 Cálculo de incertezas para o laboratório 4 a $50^{\circ} \mathrm{C} \ldots \ldots$. . . . . . . . 59

B.17 Cálculo de incertezas para o laboratório 4 a $175^{\circ} \mathrm{C} \ldots \ldots$. . . . . . . . . . 59

B.18 Cálculo de incertezas para o laboratório 4 a $300^{\circ} \mathrm{C} \ldots \ldots$. . . . . . . . . . . . . . . . . . 60

B.19 Cálculo de incertezas para o laboratório 4 a $425^{\circ} \mathrm{C} \ldots \ldots$. . . . . . . . . . . 60

B.20 Cálculo de incertezas para o laboratório 5 a $25^{\circ} \mathrm{C} \ldots \ldots$. . . . . . . . . . . . . . . . . 60

B.21 Cálculo de incertezas para o laboratório 5 a $50^{\circ} \mathrm{C} \ldots \ldots$. . . . . . . . . 60

B.22 Cálculo de incertezas para o laboratório 5 a $175^{\circ} \mathrm{C} \ldots \ldots$. . . . . . . . 61

B.23 Cálculo de incertezas para o laboratório 5 a $300^{\circ} \mathrm{C} \ldots \ldots$. . . . . . . . . . 61

B.24 Cálculo de incertezas para o laboratório 5 a $425^{\circ} \mathrm{C} \ldots \ldots$. . . . . . . . . 61

B.25 Cálculo de incertezas para o laboratório 6 a $25^{\circ} \mathrm{C} \ldots \ldots$. . . . . . . . . . . . . . 61

B.26 Cálculo de incertezas para o laboratório 6 a $50^{\circ} \mathrm{C} \ldots \ldots 2$

B.27 Cálculo de incertezas para o laboratório 6 a $175^{\circ} \mathrm{C} \ldots \ldots$. . . . . . . . . . . . . . . . . 62

B.28 Cálculo de incertezas para o laboratório 6 a $300^{\circ} \mathrm{C} \ldots \ldots$. . . . . . . . . 62

B.29 Cálculo de incertezas para o laboratório 6 a $425^{\circ} \mathrm{C} \ldots \ldots$. . . . . . . . . . 62

B.30 Cálculo de incertezas para o laboratório 7 a $25^{\circ} \mathrm{C} \ldots \ldots$. . . . . . . . . . . . . . . . . . . . . . 63

B.31 Cálculo de incertezas para o laboratório 7 a $50^{\circ} \mathrm{C} \ldots \ldots$. . . . . . . . . 63

B.32 Cálculo de incertezas para o laboratório 7 a $175^{\circ} \mathrm{C} \ldots \ldots$. . . . . . . . . 63

B.33 Cálculo de incertezas para o laboratório 7 a $300^{\circ} \mathrm{C} \ldots \ldots$. . . . . . . . 63

B.34 Cálculo de incertezas para o laboratório 7 a $425^{\circ} \mathrm{C} \ldots \ldots$. . . . . . . . . . 64

B.35 Cálculo de incertezas para o laboratório 8 a $25^{\circ} \mathrm{C} \ldots \ldots$. . . . . . . . . . 64

B.36 Cálculo de incertezas para o laboratório 8 a $50^{\circ} \mathrm{C} \ldots \ldots$. . . . . . . . . . 64

B.37 Cálculo de incertezas para o laboratório 8 a $175^{\circ} \mathrm{C} \ldots \ldots$. . . . . . . . . . . 64

B.38 Cálculo de incertezas para o laboratório 8 a $300^{\circ} \mathrm{C} \ldots \ldots$. . . . . . . . . . 65

B.39 Cálculo de incertezas para o laboratório 8 a $425^{\circ} \mathrm{C} \ldots \ldots$. . . . . . . . . . 65

B.40 Cálculo de incertezas para o laboratório 9 a $25^{\circ} \mathrm{C} \ldots \ldots$. . . . . . . . . 65

B.41 Cálculo de incertezas para o laboratório 9 a $50^{\circ} \mathrm{C} \ldots \ldots . \ldots . \ldots$

B.42 Cálculo de incertezas para o laboratório 9 a $175^{\circ} \mathrm{C} \ldots \ldots$. . . . . . . . . . . . . . . . . . 66

B.43 Cálculo de incertezas para o laboratório 9 a $300^{\circ} \mathrm{C} \ldots \ldots$. . . . . . . . . 66

B.44 Cálculo de incertezas para o laboratório 9 a $425^{\circ} \mathrm{C} \ldots \ldots$. . . . . . . . . . . . . . . . . 66

B.45 Cálculo de incertezas para o laboratório 10 a $25^{\circ} \mathrm{C} \ldots \ldots$. . . . . . . . 66

B.46 Cálculo de incertezas para o laboratório 10 a $50^{\circ} \mathrm{C} \ldots \ldots$. . . . . . . . . 67

B.47 Cálculo de incertezas para o laboratório 10 a $175^{\circ} \mathrm{C} \ldots \ldots$. . . . . . . . . . 67

B.48 Cálculo de incertezas para o laboratório 10 a $300^{\circ} \mathrm{C} \ldots \ldots$. . . . . . . . . 67

B.49 Cálculo de incertezas para o laboratório 10 a $425^{\circ} \mathrm{C} \ldots \ldots$. . . . . . . . . . . . . . . . . . . . 67

B.50 Cálculo de incertezas para o laboratório 11 a $25^{\circ} \mathrm{C} \ldots \ldots$. . . . . . . . . 68

B.51 Cálculo de incertezas para o laboratório 11 a $50^{\circ} \mathrm{C} \ldots \ldots$. . . . . . . . . . . . 68

B.52 Cálculo de incertezas para o laboratório 11 a $175^{\circ} \mathrm{C} \ldots \ldots$. . . . . . . . . 68

B.53 Cálculo de incertezas para o laboratório 11 a $300^{\circ} \mathrm{C} \ldots \ldots$. . . . . . . . . . . . . . . . . . . 68

B.54 Cálculo de incertezas para o laboratório 11 a $425^{\circ} \mathrm{C} \ldots$. . . . . . . . . . . . 69 


\section{Capítulo 1}

\section{Introdução}

Um componente chave para o controle de qualidade na indústria é a confiança nos dados por meio de sistemas de medição. Um sistema de medição é um processo que combina instrumentos, operadores e métodos para obter o valor de uma quantidade a ser medida, isto é, o mensurando. Em geral, o resultado de uma medição é somente uma aproximação ou estimativa do valor do mensurando, e assim, deve estar acompanhada pela incerteza dessa estimativa. A incerteza do resultado de uma medição reflete a falta de conhecimento do valor do mensurando. Como conseqüência, é importante monitorar a consistência e a competência dos laboratórios para realizarem medições específicas.

Atualmente o Brasil é um desenvolvedor de motores para automóveis e caminhões. Durante o desenvolvimento, os motores são testados em bancos de prova que simulam o carro ou o caminhão.

Na condução do ensaio, diversas características do motor são acompanhadas por sensores. As medições realizadas por estes sensores são digitalizadas e enviadas para um banco de automação e posteriormente, para um computador.

Como estes laboratórios de ensaio vendem as medições realizadas pelos sensores, a confiabilidade destas medições é fundamental. Neste sentido, reunimos os principais laboratórios do país para participarem de um ensaio de proficiência. Entre os diversos sensores utilizados pelos laboratórios, vamos considerar os sensores de temperatura denominados termopares.

Ensaios de Proficiência (EP) é o uso de ensaios de comparações interlaboratoriais para determinar o desempenho dos laboratórios para ensaios (ou calibrações) específicos e para monitorar a consistência e compatibilidade dos dados do laboratório [ver ISO Guia 43]. Ensaios de comparações interlaboratoriais são conduzidos não somente para analisar laboratórios, como também para avaliar métodos e padrões.

Neste trabalho, vamos considerar o EP de esquemas de comparação de medição, onde um único item de teste é distribuído seqüencialmente entre os participantes, com os participantes enviando o item para o próximo laboratório ou enviando ao coordenador para manutenção. Os participantes devem apresentar os resultados de suas medições e suas respectivas incertezas expandidas para serem submetidas à análise.

Para analisar os resultados vamos propor:

a. Um modelo para explicar os dados, e a partir dele;

b. Encontrar as estimativas de máxima verossimilhança para os parâmetros, e então;

c. Desenvolver uma estratégia para verificar a competência dos laboratórios individualmente.

Na maioria dos trabalhos em que os erros de medição são incorporados ao modelo, ver Grubbs (1948 e 1973), Mandel (1959), Smith (1950) e Thompson (1963), a preocupação está 
nos parâmetros de variabilidade associados à medição. Além disso, alguns autores, como Fuller (1987) e Cheng \& Van Ness (1999), utilizam os modelos de regressão com erro nas variáveis para incorporar os erros de medição. Aqui, estamos interessados nos parâmetros de tendência aditiva e tendência multiplicativa, e vamos incorporar os erros de medição como proposto por Jaech (1985).

Jaech (1985) estabeleceu e desenvolveu métodos para estimar e testar as variâncias em sistemas de medição. Aqui, estamos interessados em estimar e testar hipóteses sobre o parâmetro de tendência aditiva $(\underline{\alpha})$ e sobre a tendência multiplicativa $(\underline{\beta})$ com respeito ao valor de referência. Aqui, os parâmetros de variância são estimados por um processo de calibração descrito no Capítulo 2. Portanto, nós iremos encontrar aproximações para as estimativas de máxima verossimilhança dos parâmetros $\underline{\alpha}$ e $\underline{\beta}$ do laboratório com respeito ao valor de referência e desenvolver uma estratégia para avaliarmos a consistência das medições dos laboratórios. Para ilustrar, vamos analisar os dados obtidos pelo grupo de ensaios, cujo sensor de motores é um termopar pt-100 com indicador de temperatura.

\section{$1.1 \quad$ Objetivo}

Considere um programa de comparação interlaboratorial com um grupo de $N$ laboratórios. Estes laboratórios vão medir um mesmo equipamento padrão, devidamente calibrado por um laboratório reconhecido internacionalmente. Os laboratórios participantes realizam as medições do equipamento padrão e emitem um certificado com os valores medidos e a incerteza associada (desvio padrão).

Nossa metodologia de análise irá comparar os resultados dos laboratórios em relação a um valor de referência, para determinar se algum laboratório participante está com problemas na medição. Estes problemas são devidos a falhas nos equipamentos utilizados na medição, operadores que não seguem corretamente os procedimentos de medição, entre outros.

Aqui, propomos um modelo estatístico para os dados provenientes do ensaio de proficiência conduzido pelo grupo de ensaios de motores. Através deste modelo, vamos avaliar a compatibilidade dos resultados do grupo de laboratórios participantes.

\subsection{Organização do Trabalho}

Em relação a sua organização, este trabalho foi estruturado em cinco capítulos.

Neste capítulo, foram abordadas, de uma forma sucinta, as definições relativas ao entendimento sobre Ensaios de Proficiência.

No capítulo 2, são apresentados os dados referentes ao programa de EP conduzido pelo Grupo de Motores na área de temperatura e uma análise gráfica das tendências dos laboratórios.

No capítulo 3, nós definimos o modelo utilizado para explicar os dados, apresentamos um algoritmo para determinarmos aproximações para as estimativas de máxima verossimilhança dos parâmetros e um estudo sobre a convergência do algoritmo EM.

No capítulo 4, apresentamos as aproximações para as estimativas de máxima verossimilhança para os parâmetros do nosso modelo obtidas via algoritmo EM e os intervalos de confiança para os parâmetros obtidos via simulação.

No capítulo 5 apresentamos as conclusões e propostas futuras. 


\section{Capítulo 2}

\section{Apresentação dos Dados e Avaliação das Incertezas de Medição}

\subsection{Introdução}

Neste capítulo, apresentaremos os dados relativos ao programa de EP conduzido pelo Grupo de Ensaio de Motores. Além disso, vamos fazer uma introdução ao conceito de incerteza e mostrar como os laboratórios aplicaram este conceito para estimar a variância de suas medições.

\subsection{Apresentação dos dados}

Buscando a comprovação da competência técnica para realizarem suas medições, vários laboratórios do Estado de São Paulo se comprometeram a participar do programa de comparação interlaboratorial por ensaios de proficiência, conduzido pelo Grupo de Ensaios de Motores. Em uma comparação interlaboratorial, os diversos laboratórios medem o mesmo equipamento padrão. No nosso caso, os laboratórios participantes realizaram três medições em cinco faixas de temperatura, $25^{\circ} \mathrm{C}, 50^{\circ} \mathrm{C}, 175^{\circ} \mathrm{C}, 300^{\circ} \mathrm{C}$ e $425^{\circ} \mathrm{C}$ utilizando um termopar com indicador de temperatura.

Estas medições foram realizadas utilizando-se um termopar do próprio laboratório e um termopar padrão (que circula entre os laboratórios participantes do EP), que indicaremos por artefato. O procedimento para a realização da comparação é simples. Ambos os termopares são colocados no forno e as diferenças entre as medições são registradas.

Para a coleta dos dados, o experimento foi realizado seguindo o seguinte procedimento:

- Elevou-se a temperatura do forno e registrou-se a temperatura indicada por cada termopar nas faixas de temperatura mencionadas acima;

- Abaixou-se a temperatura do forno e anotou-se novamente as temperaturas indicadas;

- Elevou-se novamente a temperatura do forno e fez-se outro registro das temperaturas. 


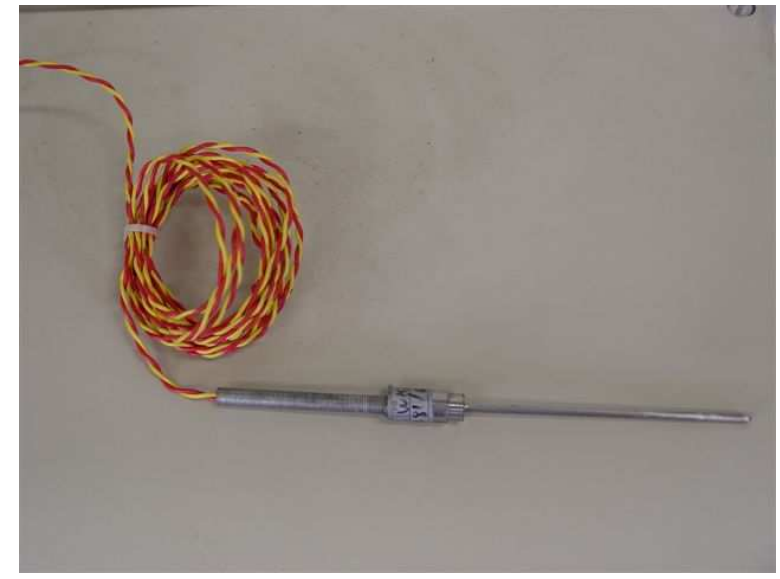

Figura 2.1: Termopar

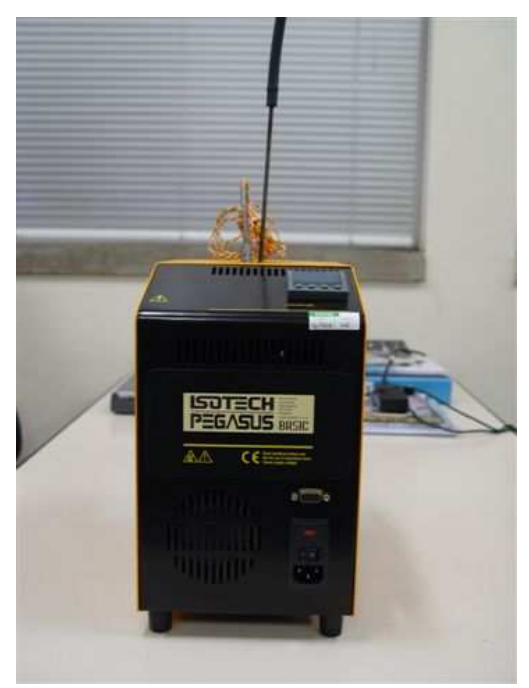

Figura 2.2: Forno

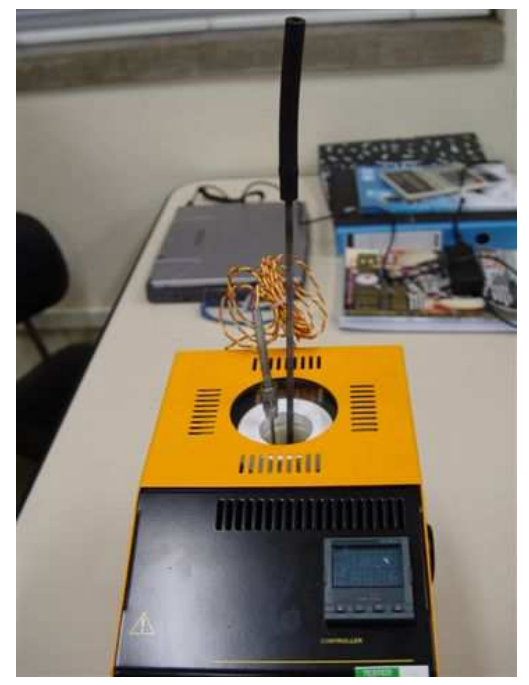

Figura 2.3: Ensaio 
Para a análise dos dados, vamos trabalhar com a diferença entre a diferença das medições do artefato e o equipamento do laboratório, e o valor de referência ou seja, definimos como tendência o resultado

(Medição do Artefato - Medição do Equipamento do Laboratório) - (Valor de Referência), (2.1) pois desta forma poderemos fazer uma melhor comparação entre os laboratórios. Na realidade, o forno tem uma exatidão de $\pm 3^{\circ} \mathrm{C}$. Assim, ao ajustarmos o forno para $300^{\circ} \mathrm{C}$, este deve estabilizar em uma temperatura no intervalo de $\left(297^{\circ} \mathrm{C}, 303^{\circ} \mathrm{C}\right)$. Desta forma, para compararmos as medições dos laboratórios devemos utilizar a transformação 2.1. A comparação será feita entre o resultado acima contra os patamares de temperatura.

Os laboratórios Salcas e Consistec são considerados laboratórios de referência, pois participam de vários programas de EP internacional e pertencem à Rede Brasileira de Calibração (RBC). Assim, vamos tomar as medições desses laboratórios como referência para nosso EP.

Os laboratórios participantes e o percurso do padrão estão descritos na Tabela 2.2. Também apresentamos na Tabela 2.1 os dados referentes às medições dos laboratórios padrão Salcas e Consistec, contendo as médias das diferenças entre as medições realizadas em cada laboratório e desvios padrão correspondentes aos patamares de temperatura $25^{\circ} \mathrm{C}, 50^{\circ} \mathrm{C}$, $175^{\circ} \mathrm{C}, 300^{\circ} \mathrm{C}$ e $425^{\circ} \mathrm{C}$.

\begin{tabular}{|c|c|c|c|c|}
\hline Temperatura & $\begin{array}{c}\text { Tendência } \\
\text { Salcas (TS) }\end{array}$ & $\begin{array}{c}\text { Tendência } \\
\text { Consistec (TC) }\end{array}$ & Valor de Referência & Desvio Padrão \\
\hline 25 & & 1,7 & 1,7 & 0,1 \\
\hline 50 & 1,6 & 1,7 & 1,65 & 0,2015565 \\
\hline 175 & 1,7 & 1,4 & 1,55 & 0,2015565 \\
\hline 300 & 1,2 & 1,2 & 1,2 & 0,3640055 \\
\hline 425 & 1,2 & 1,1 & 1,15 & 0,3640055 \\
\hline
\end{tabular}

Tabela 2.1: Dados dos laboratórios de referência Salcas e Consistec $\left(\mathrm{em}^{\circ} \mathrm{C}\right)$

\begin{tabular}{|c|c|}
\hline Roteiro & Laboratório \\
\hline \hline 1 & GM Powertrain Ltda \\
\hline 2 & Robert Bosh Ltda \\
\hline 3 & Delphi Automotive Systems \\
\hline 4 & Garrett \\
\hline 5 & Mahle - Mogi Guaçu \\
\hline 6 & Mahle - São Paulo \\
\hline 7 & Instituto Mauá de Tecnologia \\
\hline 8 & MWM Motores Diesel \\
\hline 9 & OMG Brasil Ldta \\
\hline 10 & Magneti Marelli Controle Motor \\
\hline 11 & \\
\hline
\end{tabular}

Tabela 2.2: Roteiro seguido pelo artefato

Portanto, após a realização das medições no artefato e no seu respectivo equipamento, todos os laboratórios enviaram seus relatórios ao coordenador estatístico, contendo as informações necessárias para a análise, conforme as Tabelas 2.3 e A.1 a A.10 (Apêndice A). Nestas tabelas, apresentamos os dados obtidos no programa de EP descrito acima, onde onze laboratórios realizaram três medições em um mesmo artefato, um termopar com indicador de temperatura. 
Nas tabelas a seguir,

- $F \cdot M\left({ }^{\circ} C\right)$ : faixa de medição em ${ }^{\circ} C$;

- A1 é o avanço de temperatura;

- R1 é o retorno da temperatura;

- A2 é o segundo avanço de temperatura;

- Art.: temperaturas registradas pelo artefato;

- E.M.L: temperaturas registradas pelo termopar do laboratório ;

- D: representa a diferença entre a medição do artefato e o equipamento do laboratório;

- med/tend: média de D;

- DPM: desvio padrão da média;

- UE T e UE Ind.: incertezas expandidas do termopar e do indicador de temperatura, respectivamente;

- K T e K Ind.: fatores de abrangência do termopar e do indicador de temperatura, respectivamente.

Nas seções seguintes serão discutidos os conceitos de incerteza expandida e fator de abrangência. Para exemplificar, apresentaremos somente a tabela com os dados do laboratório 1. As tabelas contendo os dados dos demais laboratórios encontram-se no Apêndice A.

\begin{tabular}{|c|c|c|c|c|c|c|c|c|c|c|c|c|c|c|c|c|}
\hline \multicolumn{17}{|c|}{ Calibração } \\
\hline $\begin{array}{l}\text { F.M. } \\
\left({ }^{\circ} \mathrm{C}\right)\end{array}$ & Art. & $\begin{array}{l}1 \\
\text { E.M.L. }\end{array}$ & $\mathrm{D}$ & Art. & $\begin{array}{l}1 \\
\text { E.M.L. }\end{array}$ & $\mathrm{D}$ & Art. & $\begin{array}{l}2 \\
\text { E.M.L. }\end{array}$ & $\mathrm{D}$ & $\begin{array}{c}\text { med / } \\
\text { tend }\end{array}$ & $\begin{array}{l}\text { Desvio } \\
\text { Padrão }\end{array}$ & DPM & $\begin{array}{c}\mathrm{UE} \\
\mathrm{T}\end{array}$ & $\begin{array}{l}\mathrm{K} \\
\mathrm{T} \\
\end{array}$ & $\begin{array}{l}\text { UE } \\
\text { Ind. }\end{array}$ & $\begin{array}{c}\mathrm{K} \\
\text { Ind. }\end{array}$ \\
\hline 25 & 22,4 & 20,6 & 1,8 & 23,1 & 21,2 & 1,9 & 23,1 & 21,2 & 1,9 & 1,9 & 0,058 & 0,033 & 0,2 & 2 & 0,8 & 2 \\
\hline 50 & 55,0 & 52,7 & 2,3 & 51,0 & 49,2 & 1,8 & 50,0 & 48,3 & 1,7 & 1,9 & 0,321 & 0,186 & 0,2 & 2 & 0,8 & 2 \\
\hline 175 & 175,0 & 175,8 & $-0,8$ & 175,0 & 176,0 & $-1,0$ & 175,0 & 176,4 & $-1,4$ & $-1,1$ & 0,306 & 0,176 & 0,2 & 2 & 0,8 & 2 \\
\hline 300 & 300,0 & 301,4 & $-1,4$ & 300,0 & 302,2 & $-2,2$ & 300,0 & 302,4 & $-2,4$ & $-2,0$ & 0,529 & 0,306 & 0,7 & 2 & 0,8 & 2 \\
\hline 425 & 425,0 & 427,7 & $-2,7$ & 425,0 & 427,6 & $-2,6$ & 425,0 & 427,6 & $-2,6$ & $-2,6$ & 0,058 & 0,033 & 0,7 & 2 & 0,8 & 2 \\
\hline
\end{tabular}

Tabela 2.3: Dados do laboratório 1

Os resultados das medições que são relevantes para a comparação entre os laboratórios correspondem às diferenças entre a medição do artefato e do equipamento do laboratório.

A seguir, apresentaremos os gráficos de pontos dos dados descritos nas Tabelas 2.3 e A.1 a A.10, para cada patamar de temperatura. Como já descrito anteriormente D representa a diferença entre as medições do artefato e do equipamento do laboratório, e VR representa o valor de referência. 


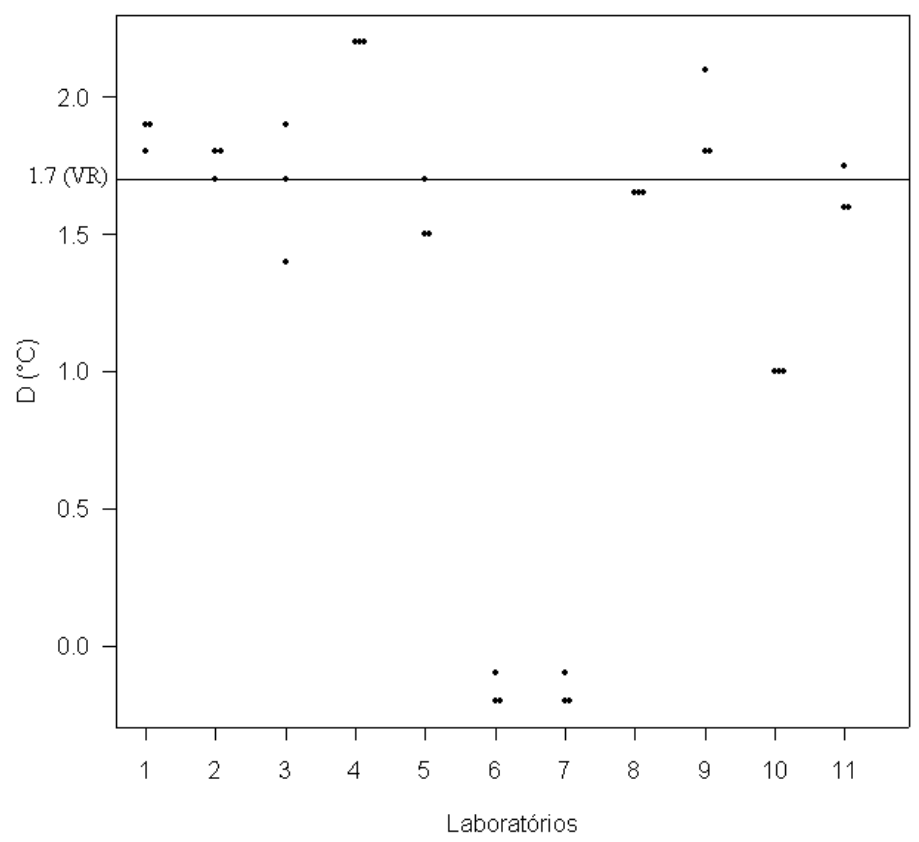

Figura 2.4: Gráfico de D a $25^{\circ} \mathrm{C}$

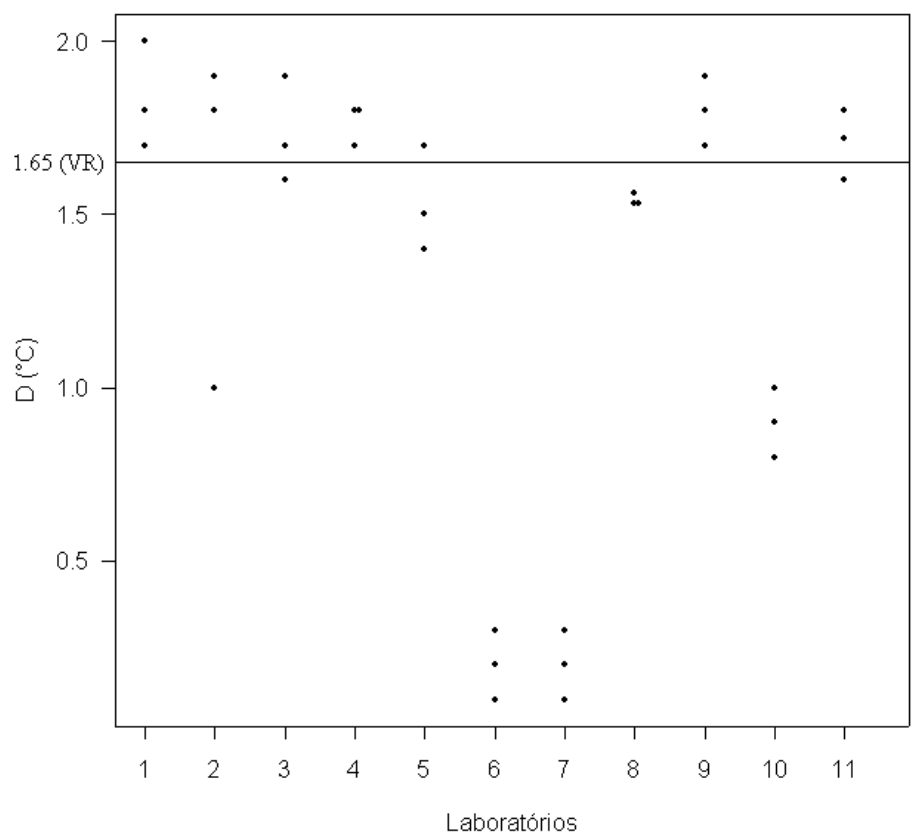

Figura 2.5: Gráfico de $\mathrm{D}$ a $50^{\circ} \mathrm{C}$ 


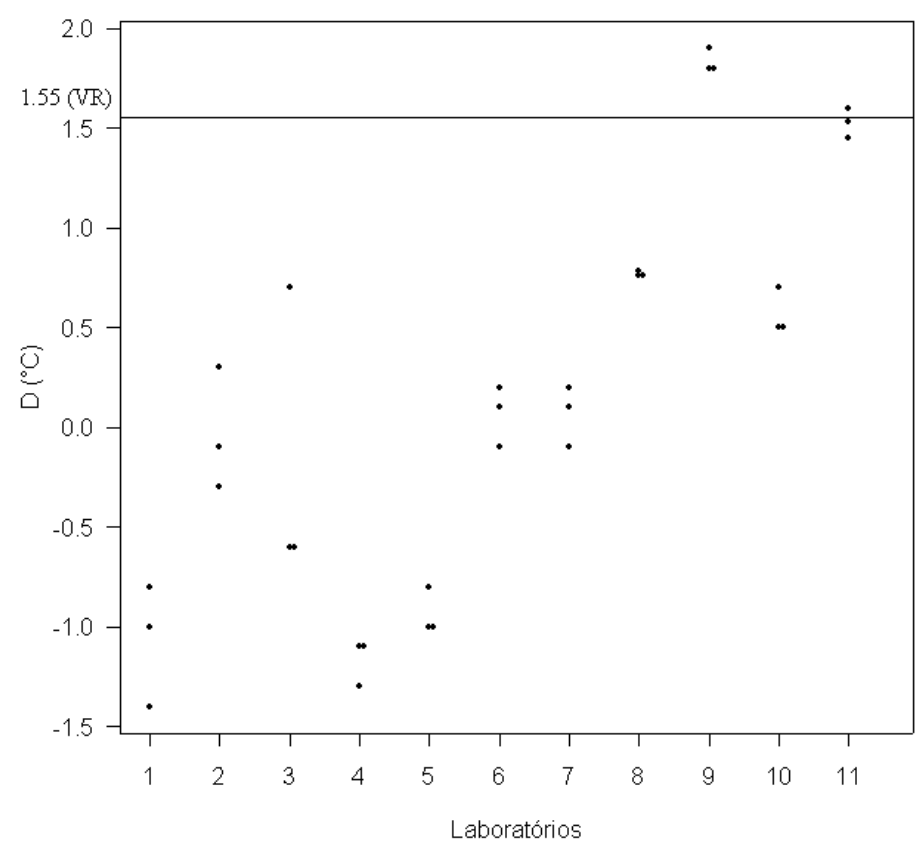

Figura 2.6: Gráfico de D a $175^{\circ} \mathrm{C}$

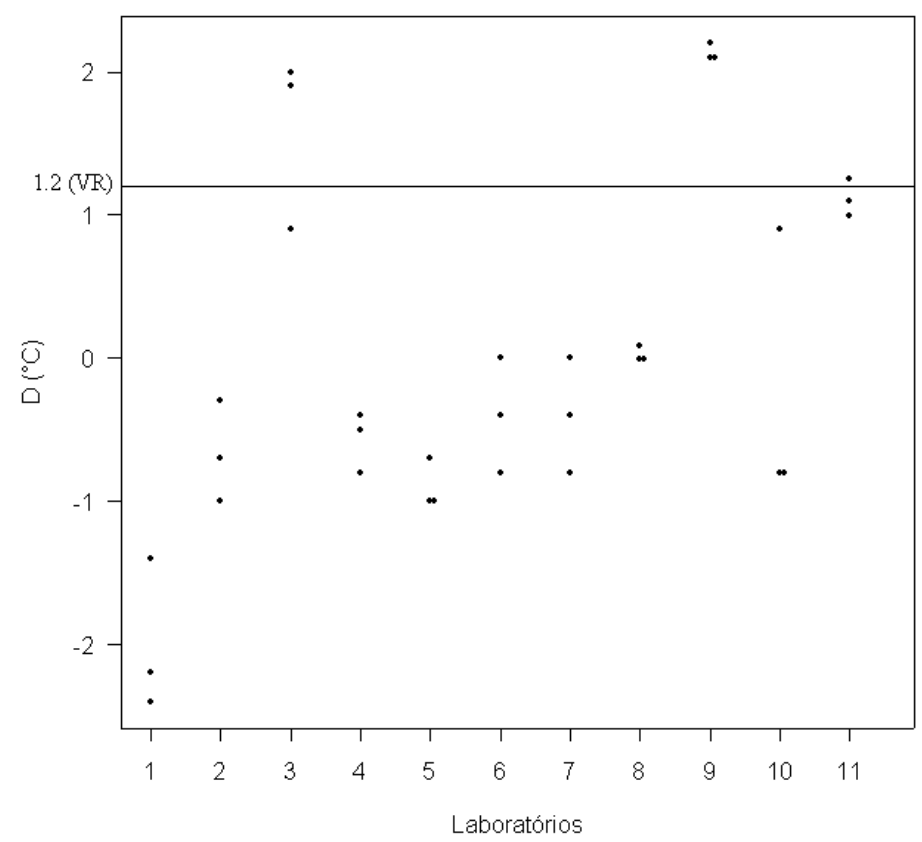

Figura 2.7: Gráfico de D a $300^{\circ} \mathrm{C}$ 


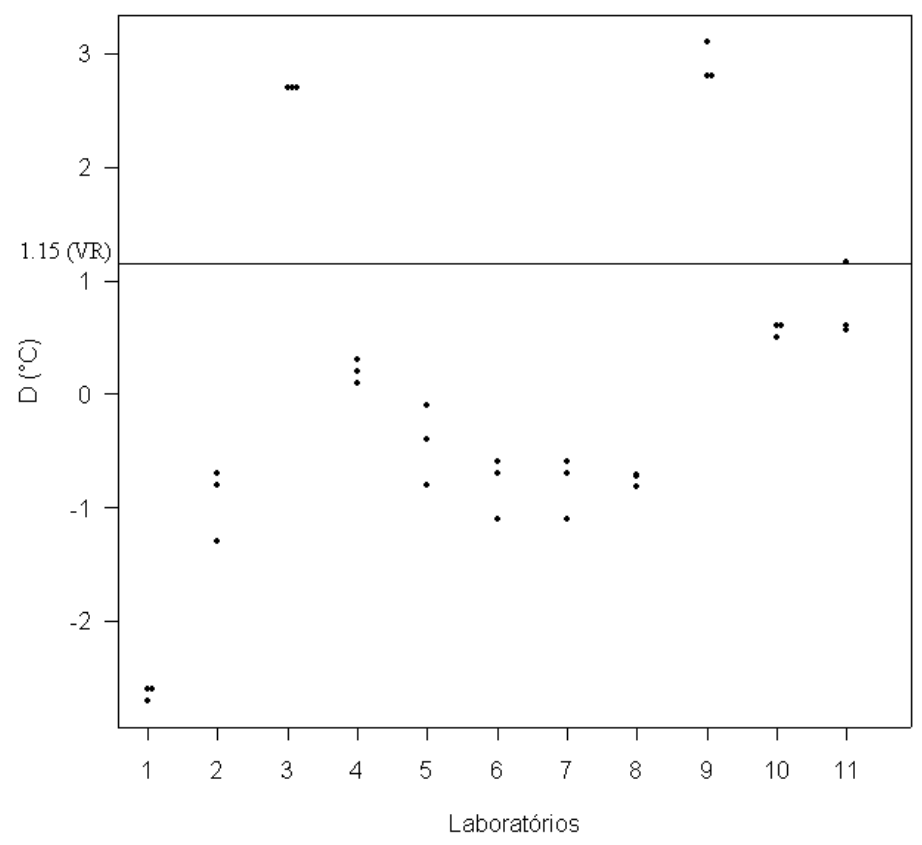

Figura 2.8: Gráfico de $\mathrm{D}$ a $425^{\circ} \mathrm{C}$

Através dos gráficos, observamos uma grande variabilidade entre as medições dos laboratórios, em todos os patamares de temperatura. Esta variabilidade depende dos equipamentos, padrões e estrutura de cada laboratório. Também podemos notar que conforme o patamar de temperatura aumenta, a diferença entre a medição do artefato e do equipamento do laboratório (D) é menor do que o valor de referência (VR).

Para comparar as medições dos laboratórios com as medições dos laboratórios de referência (Salcas / Consistec), o artefato e o equipamento do laboratório foram inseridos no mesmo forno. O forno foi programado para "estabilizar" em 5 patamares de temperatura $\left(25^{\circ} \mathrm{C}, 50^{\circ} \mathrm{C}, 175^{\circ} \mathrm{C}, 300^{\circ} \mathrm{C}\right.$ e $\left.425^{\circ} \mathrm{C}\right)$, sendo registrada a diferença de temperatura entre o artefato e o equipamento do laboratório.

A seguir, apresentamos um gráfico da tendência de cada laboratório em relação ao valor de referência. Como tendência temos a diferença entre a medição do artefato e do laboratório $(D)$ menos o valor de referência (Tabela 2.1). Na tabela abaixo exemplificamos o cálculo da tendência para o laboratório 1. 


\begin{tabular}{|c|c|c|c|}
\hline Patamar & $D$ & Valor de Referência & Tendências \\
\hline 25 & 1,8 & 1,7 & 0,1 \\
\hline 25 & 1,9 & 1,7 & 0,2 \\
\hline 25 & 1,9 & 1,7 & 0,2 \\
\hline 50 & 2,3 & 1,65 & 0,65 \\
\hline 50 & 1,8 & 1,65 & 0,15 \\
\hline 50 & 1,7 & 1,65 & 0,05 \\
\hline 175 & $-0,8$ & 1,55 & $-2,35$ \\
\hline 175 & $-1,0$ & 1,55 & $-2,55$ \\
\hline 175 & $-1,4$ & 1,55 & $-1,95$ \\
\hline 300 & $-1,4$ & 1,2 & $-2,6$ \\
\hline 300 & $-2,2$ & 1,2 & $-3,4$ \\
\hline 300 & $-2,4$ & 1,2 & $-3,6$ \\
\hline 425 & $-2,7$ & 1,15 & $-3,85$ \\
\hline 425 & $-2,6$ & 1,15 & $-3,75$ \\
\hline 425 & $-2,6$ & 1,15 & $-3,75$ \\
\hline
\end{tabular}

Tabela 2.4: Cálculo da tendência para o laboratório 1

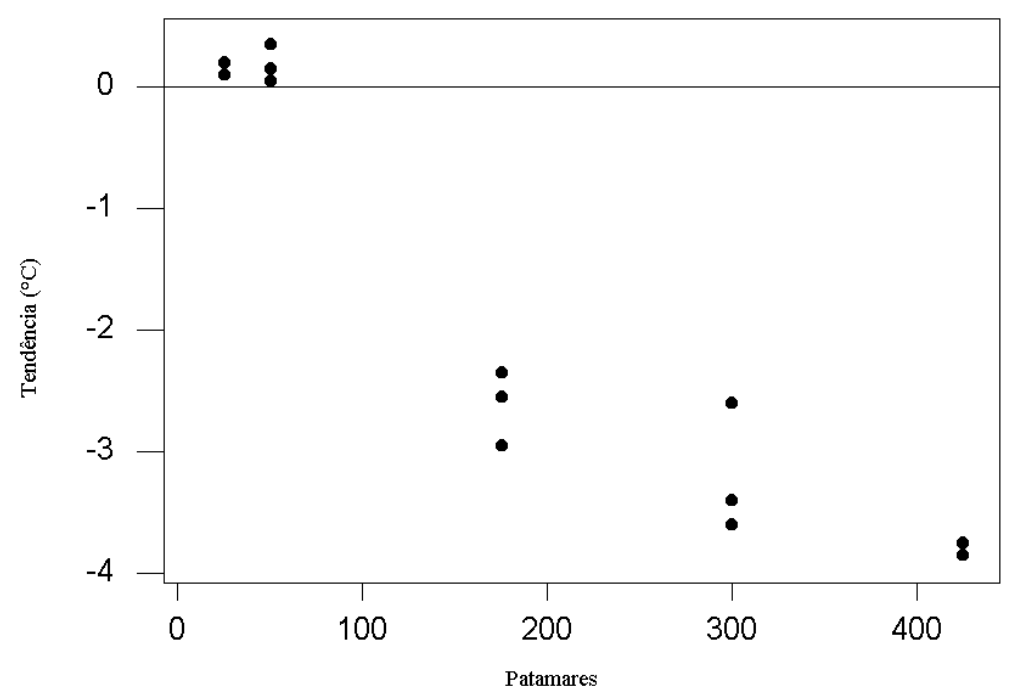

Figura 2.9: Gráfico da tendência do laboratório 1 


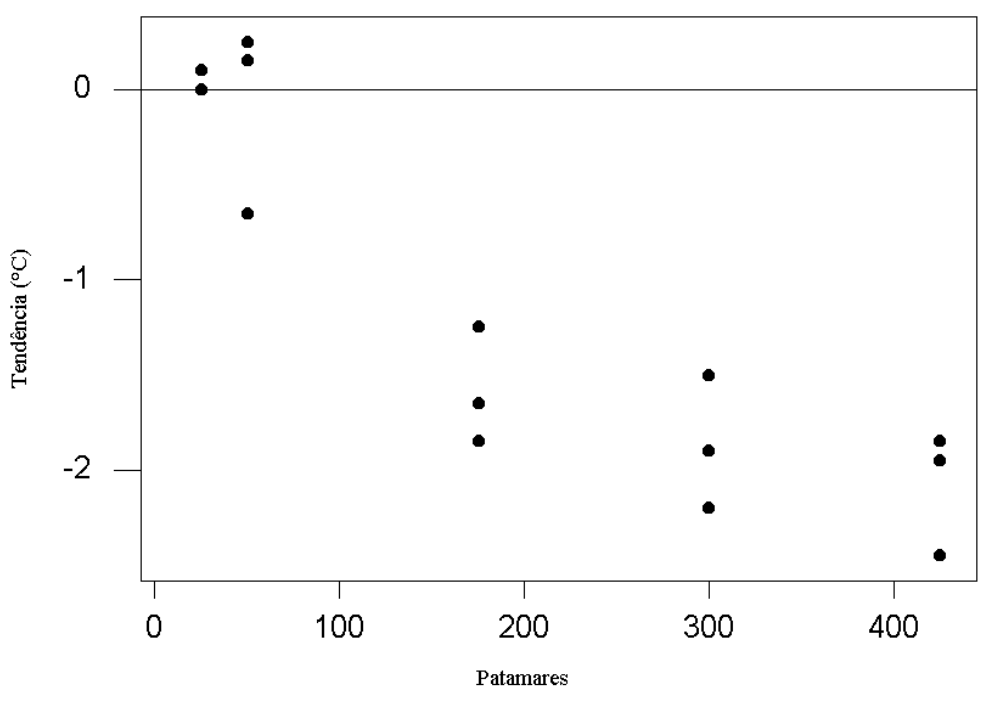

Figura 2.10: Gráfico da tendência do laboratório 2

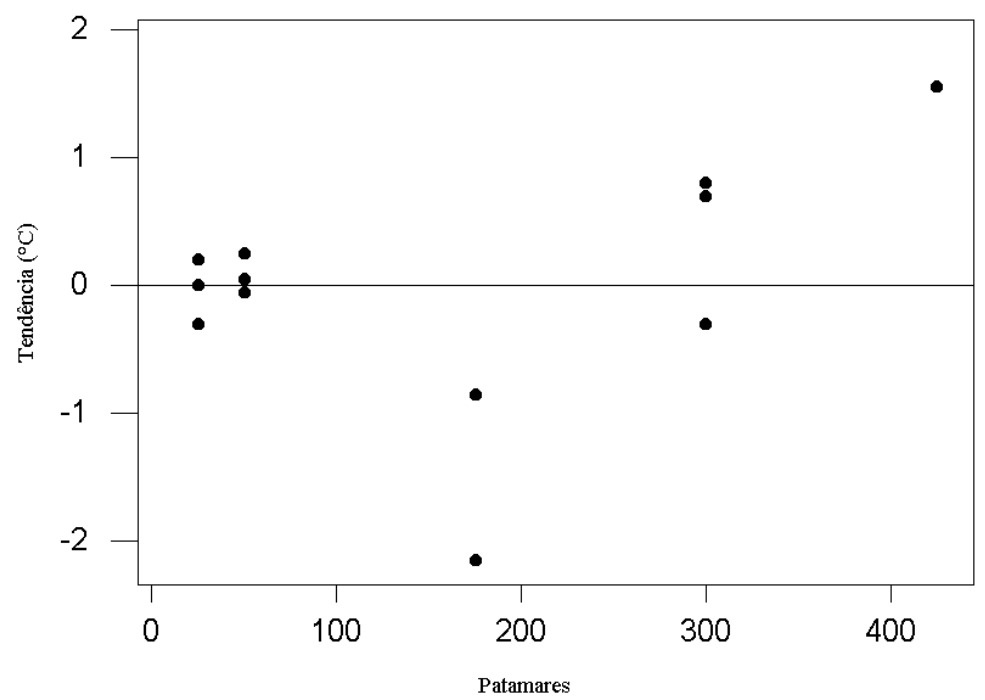

Figura 2.11: Gráfico da tendência do laboratório 3 


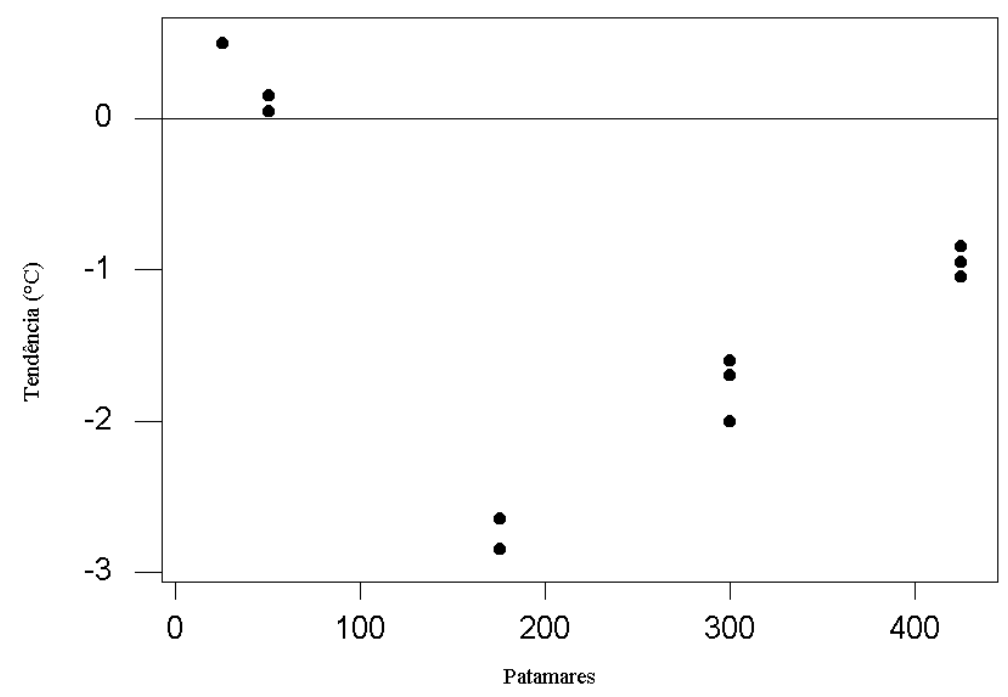

Figura 2.12: Gráfico da tendência do laboratório 4

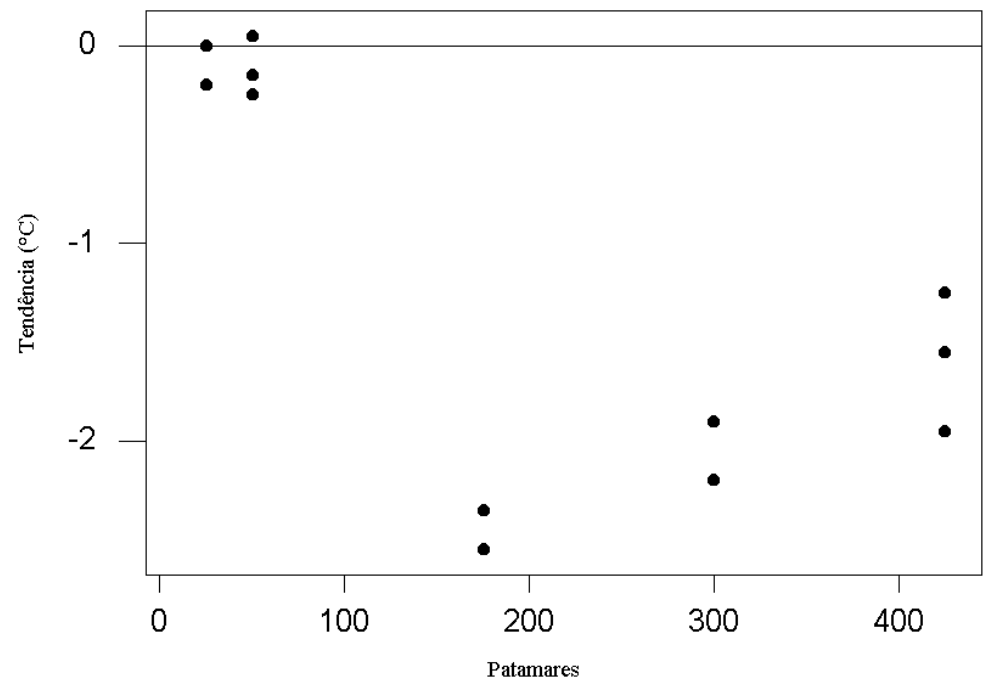

Figura 2.13: Gráfico da tendência do laboratório 5 


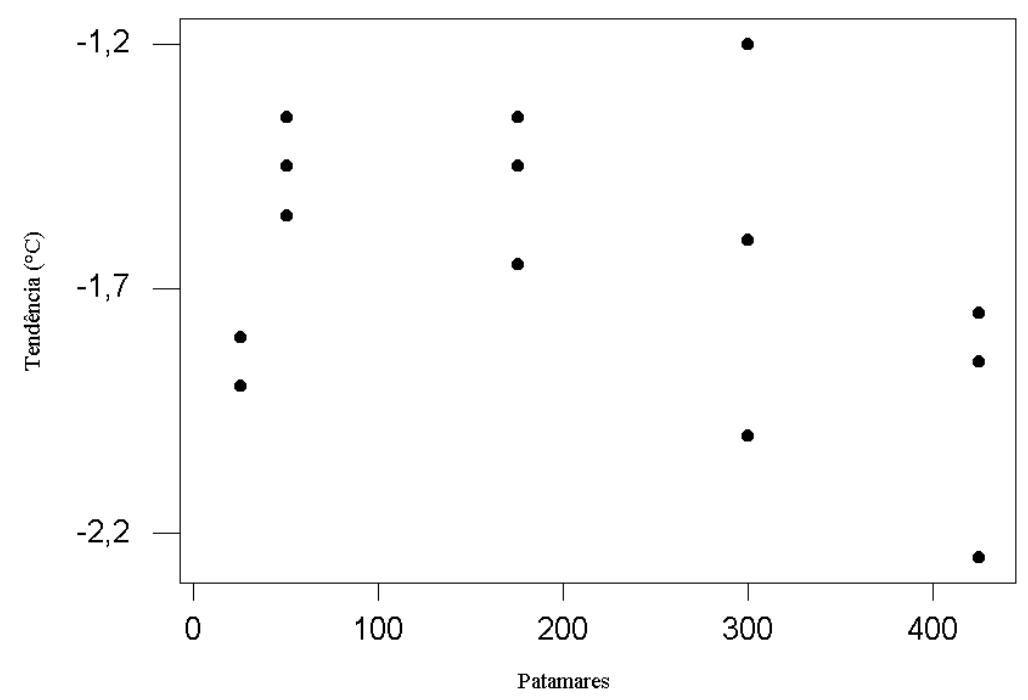

Figura 2.14: Gráfico da tendência do laboratório 6

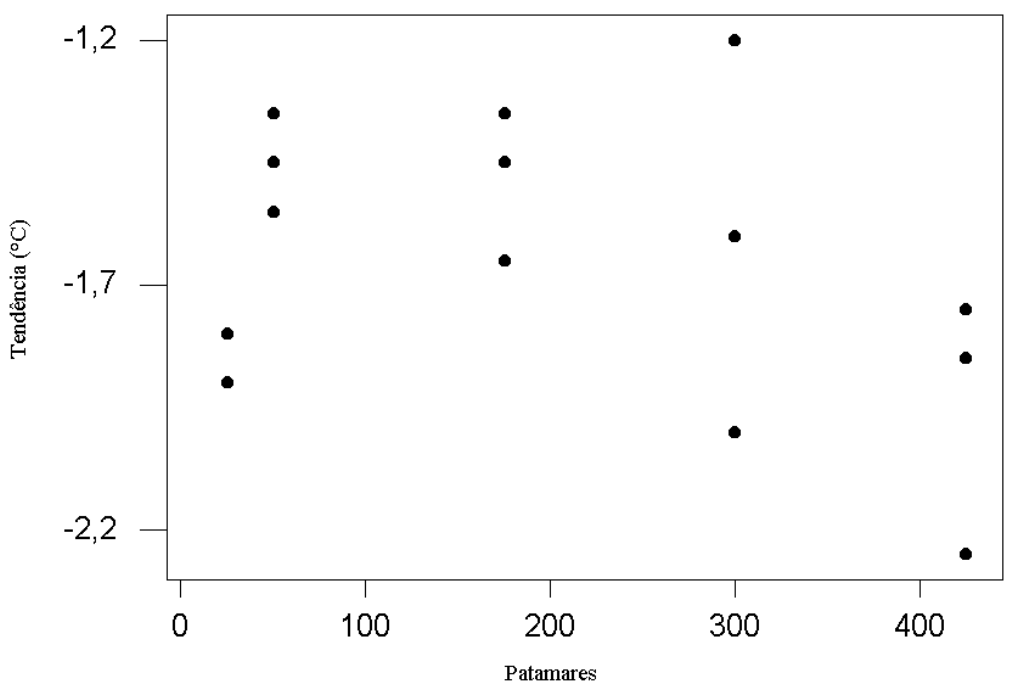

Figura 2.15: Gráfico da tendência do laboratório 7 


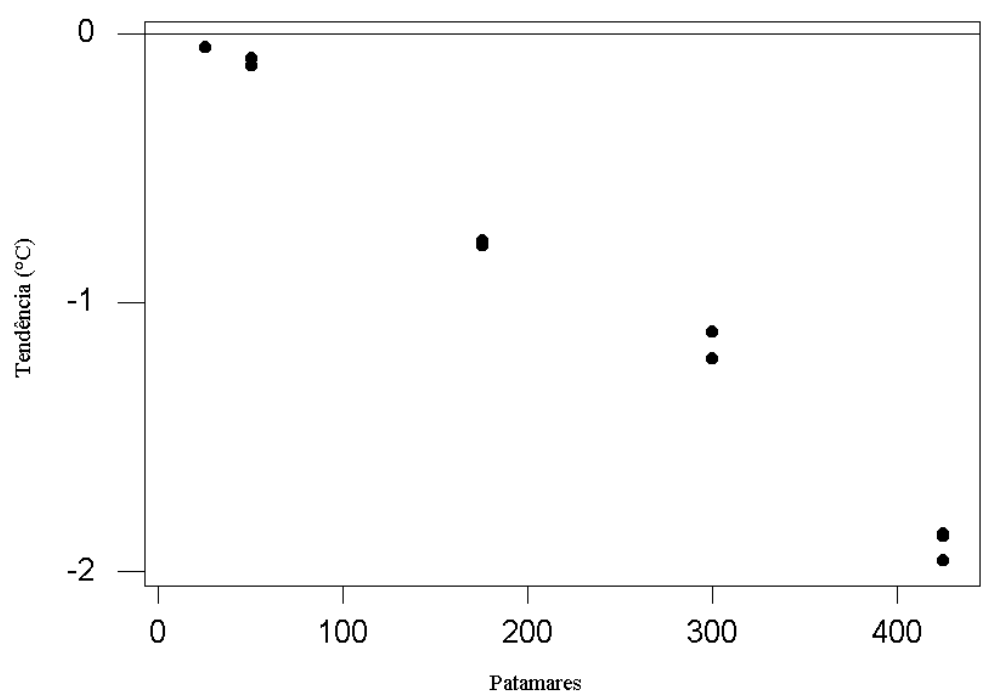

Figura 2.16: Gráfico da tendência do laboratório 8

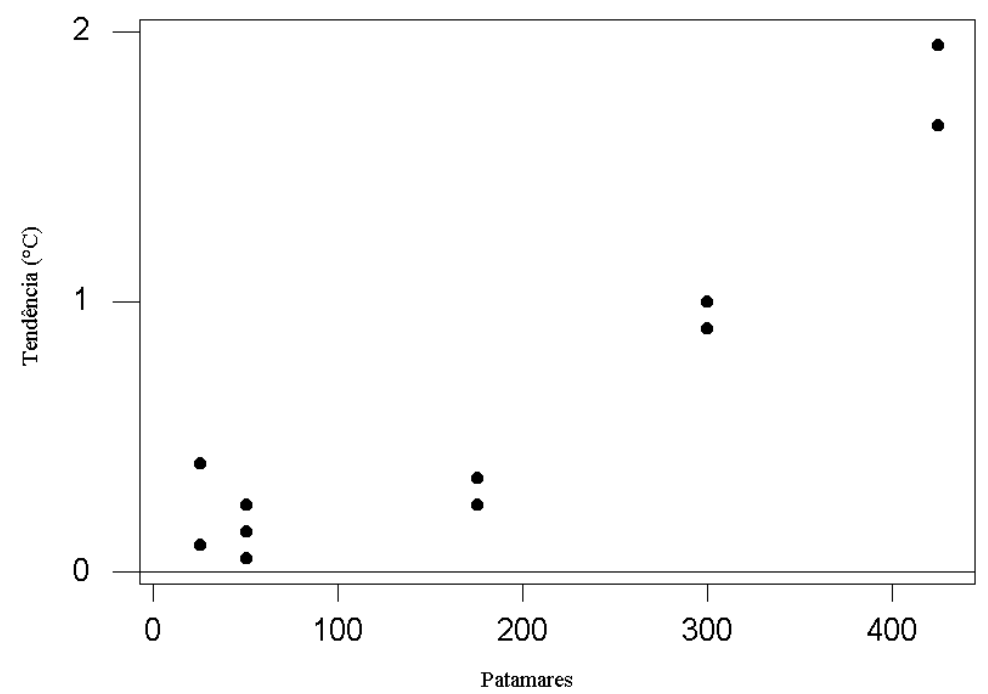

Figura 2.17: Gráfico da tendência do laboratório 9 


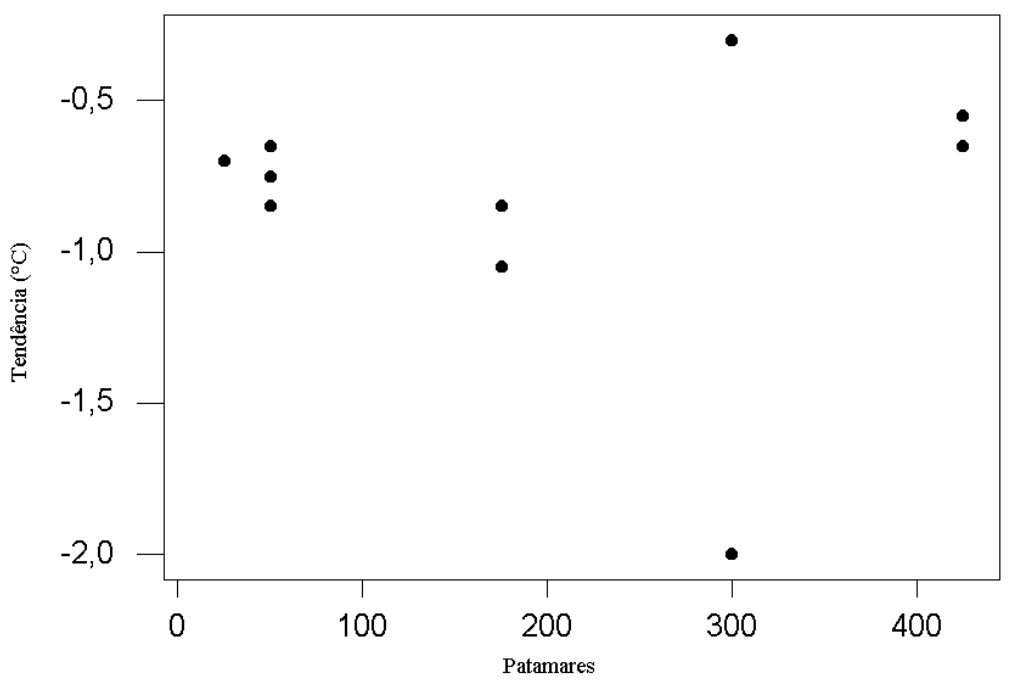

Figura 2.18: Gráfico da tendência do laboratório 10

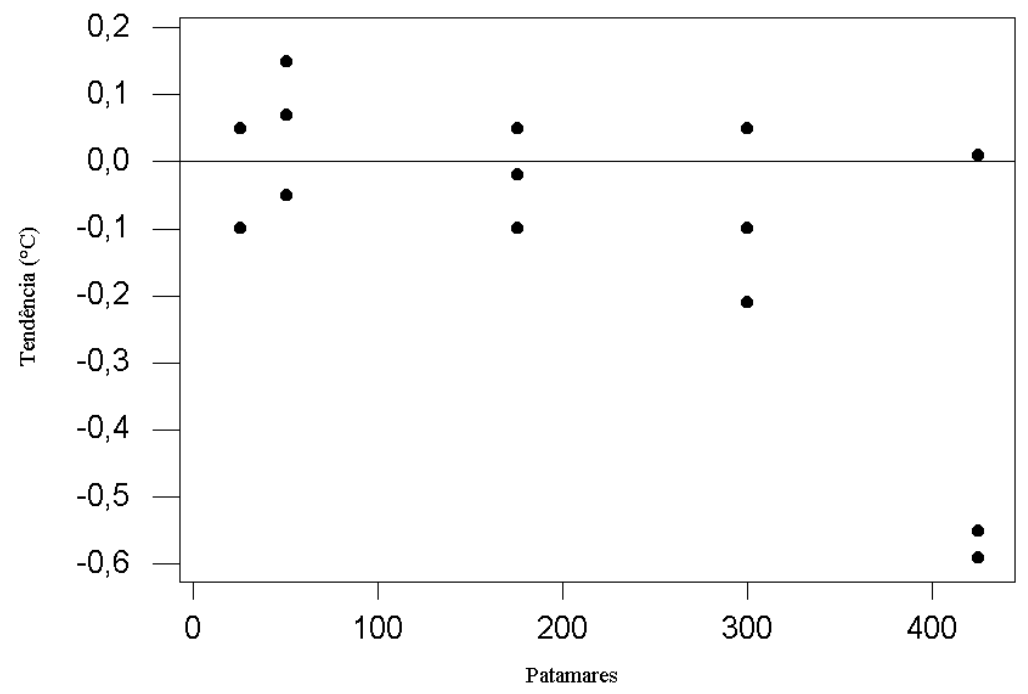

Figura 2.19: Gráfico da tendência do laboratório 11

Pelos gráficos acima podemos notar que exceto os laboratórios 3 e 9, todos os outros laboratórios possuem uma tendência negativa, a medida que o patamar de temperatura aumenta. A tendência de cada laboratório é mais apropriada para avaliarmos a consistência das medições dos laboratórios. Pois quanto mais próximo de zero a tendência, mais próximas as medições estão do valor de referência. Assim, no próximo capítulo, vamos propor um modelo de regressão apropriado para avaliar os parâmetros associados à tendência de cada laboratório (variável resposta) com o patamar de temperatura. 
Na próxima seção, apresentaremos alguns comentários sobre a recomendação, feita pelo ISO/GUM (1998), para avaliação de incertezas de medição. O termo incerteza de medição é sinônimo de desvio padrão.

\subsection{Avaliação da Incerteza de Medição}

O objetivo de uma medição é determinar o valor do mensurando, isto é, o valor da grandeza específica a ser medida. Uma medição começa, portanto, com uma especificação apropriada do mensurando, do método de medição e do procedimento de medição. Em geral, o resultado de uma medição é somente uma aproximação ou estimativa do valor do mensurando e, assim, só é completa quando acompanhada pela declaração da incerteza dessa estimativa.

Na prática, a especificação requerida ou definição requerida do mensurando é ditada pela exatidão requerida da medição (grau de concordância entre o resultado de uma medição e um valor de referência para o mensurando). O mensurando deve ser definido com totalidade suficiente relativa à exatidão requerida, de modo que, para todos os fins práticos associados com a medição, seu valor seja único.

A operação de medição é realizada, genericamente, por um sistema de medição (SM). O SM é o conjunto completo de instrumentos de medição e outros equipamentos acoplados para executar uma medição específica, ou seja, é um processo que combina instrumentos, operadores e métodos para obter o valor de uma quantidade a ser medida. Todo sistema de medição não é perfeito, ou seja, na realização de medições existem várias influências que geram erros nos resultados obtidos, de modo que não existe uma medição perfeita (sem erros).

Aspectos tecnológicos forçam que qualquer sistema de medição construído resulte imperfeito: suas dimensões, forma geométrica, material, propriedades elétricas, ópticas, pneumáticas, etc, não correspondem exatamente ao ideal. As leis e princípios físicos que regem o funcionamento de alguns sistemas de medição nem sempre são perfeitamente lineares, como uma análise simplista poderia supor. A existência de desgaste e deterioração de partes agravam ainda mais esta condição. Em todos os casos, o SM gera erros de medição.

O erro de medição é impossível de ser eliminado, porém podemos ao menos delimitálo com confiança apropriada. Mesmo sabendo da sua existência, ainda é possível obter informações confiáveis de uma medição, desde que a ordem de grandeza e a natureza deste erro sejam conhecidas. O erro é um conceito idealizado e não pode ser exatamente conhecido.

Geralmente ocorrem erros de vários tipos em uma mesma medida. Entretanto, esses diferentes tipos de erros podem ser separados em três grandes grupos:

1. Erros Aleatórios ou Erros Estatísticos: Resultam de variações aleatórias no valor medido de uma grandeza, devido a fatores que não podem ser controlados ou que por qualquer motivo não são controlados. Geralmente, essas variações se devem somente ao sistema e processo de medição, mas em alguns casos, as variações aleatórias também ocorrem na própria grandeza. Diversos fatores contribuem para o surgimento dos erros aleatórios, por exemplo, existência de folgas, vibrações, flutuações de tensão elétrica, instabilidades internas ou das condições ambientais, etc.

2. Erros Sistemáticos: É a parcela de erro sempre presente nas medições realizadas sob condições de repetitividade. Da mesma forma que os erros aleatórios, os erros sistemáticos não podem ser totalmente eliminados, mas podem ser reduzidos através de correções para compensar os seus efeitos. Os erros sistemáticos mais comuns são: 
2.1 Erros Sistemáticos Instrumentais: É o erro que resulta da calibração de um instrumento de medição.

2.2 Erros Sistemáticos Teóricos: É encontrado no uso de fórmulas teóricas aproximadas ou uso de valores aproximados para eventuais constantes físicas que sejam utilizadas.

2.3 Erros Sistemáticos Ambientais: É o erro causado devido a efeitos do ambiente sobre a medição, tais como, temperatura, pressão, umidade, aceleração da gravidade, etc.

2.4 Erros Sistemáticos Observacionais: É o erro devido a falhas ou limitação do próprio operador.

2.5 Erros Sistemáticos Residuais: São os erros que não podem ser reduzidos a um valor baixo ou para os quais não seja possível fazer correções.

3. Erros Grosseiros: Consiste de enganos que, eventualmente podem ocorrer no procedimento de medida ou na realização de cálculos.

Na grande maioria dos casos, o resultado da medição é determinado através de uma série de leituras obtidas sob condições de repetitividade. Variações obtidas nas leituras repetidas são conseqüências de fatores que afetam os resultados das leituras. Além disso, o modelo matemático da medição, que transforma as leituras repetidas no resultado da medição é crítico, pois inclui fatores que não são totalmente conhecidos. Assim, a variação obtida nas leituras repetidas e a falta de informação do modelo matemático, contribuem para a incerteza do resultado da medição.

A declaração do resultado de uma medição somente é completa se ela contiver tanto o valor atribuído ao mensurando quanto a incerteza de medição associada a este valor. A incerteza de medição é um parâmetro associado ao resultado de uma medição, que caracteriza a dispersão dos valores que podem ser razoavelmente atribuídos ao mensurando (ISO/GUM(1998)). O mensurando é a grandeza física a ser medida. Em geral, os passos para estimar a incerteza são:

a. Definir a equação de medição;

b. Identificar as componentes de incerteza;

c. Calcular a incerteza padrão das componentes;

d. Calcular a incerteza combinada;

e. Calcular os graus de liberdade efetivos;

f. Calcular a incerteza expandida.

Nas subseções seguintes, abordaremos cada passo mencionado acima.

\subsubsection{Equação de Medição}

Na maioria dos casos o mensurando Y não é medido diretamente, mas é determinado a partir de N outras grandezas $X_{1}, X_{2}, \ldots X_{N}$ através de uma relação funcional f:

$$
Y=f\left(X_{1}, X_{2}, \ldots X_{N}\right)
$$

As grandezas de entrada $X_{1}, X_{2}, \ldots X_{N}$, das quais a grandeza de saída $Y$ depende, podem ser consideradas como: 
1. Grandezas cujos valores e incertezas podem ser diretamente determinadas na medição em curso. Estes valores ou incertezas podem ser obtidos, por exemplo, de uma única observação, de observações repetidas ou de julgamento baseado na experiência, e podem envolver a determinação de correções a leituras de instrumentos e correções por conta de grandeza de influência, tais como temperatura ambiente, pressão barométrica e umidade;

2. Grandezas cujos valores e incertezas são incorporados à medição a partir de fontes externas, tais como grandezas associadas com padrões de medição calibrados, materiais de referência certificados e dados de referência obtidos de manuais técnicos.

No programa de temperatura, como método de medição, cada laboratório utilizou a seguinte relação entre temperatura, resolução e indicador de temperatura:

$$
T_{A}=T_{E M}+D+\operatorname{Res}(A)+\operatorname{Res}(E M)+I n d
$$

onde,

- $T_{A}$ : Representa a temperatura no artefato;

- $T_{E M}$ : Representa a temperatura no equipamento do laboratório;

- D: Representa a diferença de leitura entre os equipamentos (Artefato e Equipamento do laboratório);

- $\operatorname{Res}(\mathrm{A})$ : Resolução do artefato;

- Res(EM): Resolução do equipamento do laboratório;

- Ind: Representa o indicador de temperatura do laboratório participante.

Os ítens $T_{E M}, D, \operatorname{Res}(\mathrm{A}), \operatorname{Res}(\mathrm{EM})$ e Ind são as grandezas de entrada e $T_{A}$, que representa a temperatura no artefato, é a grandeza de saída. A incerteza combinada de medição associada à estimativa de saída, designado por $u(y)$, é o desvio padrão do mensurando Y e deve ser determinado a partir das estimativas $x_{1}, x_{2}, \ldots, x_{N}$ das grandezas de entrada $X_{1}, X_{2}, \ldots X_{N}$, e suas incertezas padrão associadas $u\left(x_{i}\right)$. A seguir, descrevemos métodos para calcular a incerteza de cada grandeza de entrada.

\subsubsection{Avaliação da Incerteza Padrão de Medição das Estimativas de Entrada}

A incerteza padrão de uma grandeza de entrada é dividida em dois grupos, de acordo com o método utilizado para estimar seus valores numéricos:

- Tipo A: Aquelas que são avaliadas por métodos estatísticos;

- Tipo B: Aquelas que são avaliadas por outros métodos.

Assim, uma incerteza padrão do Tipo A é obtida a partir de uma função densidade de probabilidade derivada da observação de uma distribuição de freqüência, enquanto que uma incerteza padrão do Tipo B é obtida de uma suposta função de probabilidade, previamente assumida para esta fonte de incerteza.

É importante ressaltar que toda incerteza padrão é um desvio padrão. Assim, se considerarmos a incerteza do Tipo A, temos o desvio padrão da média da série de observações da 
medição. Por outro lado, se considerarmos as incertezas do Tipo B, temos o desvio padrão da distribuição de probabilidade associada.

A incerteza padrão da grandeza de entrada $D$ é avaliada pelo Tipo A enquanto que $T_{E M}$, $\operatorname{Res}(A), \operatorname{Res}(E M)$ e Ind são avaliadas pelo Tipo B. Como fonte de incerteza do Tipo A, consideramos a repetitividade das medições. No nosso caso, cada laboratório realizou três medições do artefato em cada patamar de temperatura.

Para exemplificar os cálculos, vamos tomar os dados do laboratório 1 no patamar de $25^{\circ} \mathrm{C}$. A incerteza padrão das grandezas de entrada é estimada da seguinte forma:

- Incertezas Avaliadas pelo Tipo A: Aqui, a incerteza padrão é dada pelo desvio padrão da média da série de observações da medição de temperatura. Sejam $t_{1}, t_{2}, t_{3}$ as três medições da temperatura (ver Tabela 2.3). O desvio padrão da média é

$$
S(\bar{t})=\frac{S(t)}{\sqrt{3}}=0,033
$$

onde $S(t)=\sqrt{\frac{1}{2} \sum_{k=1}^{3}\left(t_{k}-\bar{t}\right)}=0,058$ e $\bar{t}=\frac{1}{3} \sum_{k=1}^{3} t_{k}=21$. Portanto, a incerteza padrão da repetitividade é

$$
u(D)=0,033^{\circ} \mathrm{C} .
$$

- Incertezas Avaliadas pelo Tipo B: Aqui, a incerteza padrão de uma grandeza de entrada que não tenha sido obtida através de observações repetidas é avaliada por julgamento científico, baseando-se em todas as informações disponíveis sobre a possível variabilidade dessa grandeza. O conjunto de informações pode incluir:

1. dados de medições prévias;

2. a experiência ou o conhecimento geral do comportamento e propriedades de materiais e instrumentos relevantes;

3. especificações do fabricante;

4. dados fornecidos em certificados de calibração e outros certificados;

5. incertezas relacionadas a dados de referência extraídos de manuais.

A partir disso, e considerando as grandezas de entrada para a medição da temperatura, as incertezas padrão são quantificadas da seguinte forma:

- Incerteza Padrão para $T_{E M}$ : Após várias leituras de medição da temperatura, verificou-se que a distribuição de probabilidade que melhor se ajustava era a distribuição normal, cuja média é estimada pela média das observações (no nosso exemplo, $\bar{t}=21^{\circ} \mathrm{C}$ ). O desvio padrão da temperatura no equipamento do laboratório não é estimado da variabilidade associada diretamente às medições, mas via a incerteza expandida herdada do termopar. Como veremos na seção 2.3.4, a incerteza expandida é um múltiplo do desvio padrão que abrange o semi-intervalo de $95 \%$ de probabilidade. Assim, utilizando os dados da Tabela 2.3 (coluna UE T) à $25^{\circ} \mathrm{C}$, obtemos que

$$
0,2=2 * u\left(T_{E M}\right) \Rightarrow u\left(T_{E M}\right)=0,1^{\circ} C .
$$

Portanto, a incerteza padrão do padrão do laboratório é $u\left(T_{E M}\right)=0,1^{\circ} \mathrm{C}$.

A incerteza expandida do equipamento padrão do laboratório está declarada no certificado de calibração do respectivo equipamento. 
- Incerteza Padrão para Res $(A)$ : Para esta grandeza temos a informação que o termopar tem uma resolução de $0,1^{\circ} C$, ou seja, a escala de medição está dividida em intervalos de $0,1^{\circ} C$. Sendo assim, vamos considerar que $\operatorname{Res}(A)$ tem distribuição uniforme com base igual à resolução do termopar. Portanto, a incerteza padrão da $\operatorname{Res}(A)$ é

$$
u(\operatorname{Res}(A))=\frac{0,1}{\sqrt{12}}=0,028867527^{\circ} \mathrm{C} .
$$

- Incerteza Padrão para $\operatorname{Res}(E M)$ : Assim como Res $(A)$, Res(EM) também é considerada uma variável aleatória com distribuição uniforme, com a mesma resolução de $0,1^{\circ} C, \log \mathrm{o}, u(\operatorname{Res}(E M))=u(\operatorname{Res}(A))$.

- Incerteza Padrão para Ind: Após várias leituras de medição da temperatura, verificou-se que a distribuição de probabilidade que melhor se ajustava era a distribuição normal, cujo desvio padrão é estimado via a incerteza expandida herdada do indicador de temperatura. Assim, utilizando os dados da Tabela 2.3 (coluna UE Ind), à $25^{\circ} C$, obtemos que

$$
0,8=2 * u(\text { Ind }) \Rightarrow u(\text { Ind })=0,4
$$

Mais uma vez, a incerteza expandida do indicador de temperatura está declarada no seu certificado de calibração. Na próxima seção, faremos a combinação de todas as incertezas padrão calculadas acima.

\subsubsection{Avaliação da Incerteza Padrão Combinada}

A incerteza combinada é a combinação entre todas as incertezas padrão das componentes relacionadas pela equação de medição, ou seja, $u_{c}(y)$ corresponde ao desvio padrão da estimativa da grandeza de saída.

Em alguns estudos de calibração, podemos ter que duas ou mais grandezas de entrada sejam correlacionadas, e essa correlação é de certa forma uma contribuição à incerteza combinada. Para julgar se existe ou não essa correlação, é preciso conhecer o processo de medição e ter conhecimento de causa dessa dependência mútua entre as grandezas de entrada. Não tomar conhecimento dessas correlações pode levar a avaliações incorretas da incerteza padrão do mensurando.

Em alguns casos, essa correlação pode ser eliminada pela escolha apropriada da relação funcional, mas na prática, algumas grandezas são freqüentemente relacionadas, pois na avaliação de seus valores é utilizado o mesmo padrão de referência, instrumento de medição, dados de referência, ou até, o método de medição.

Nos casos onde existe a correlação, o quadrado da incerteza padrão combinada (ISO GUM, 1998, pg. 21, item 5.2.2) pode ser aproximado por

$$
u_{c}^{2}(y)=\sum_{i=1}^{N} c_{i}^{2} u^{2}\left(x_{i}\right)+2 \sum_{i=1}^{N-1} \sum_{h=i+1}^{N} c_{i} c_{h} u\left(x_{i}, x_{h}\right),
$$

onde $c_{i}$ e $c_{h}$ são os coeficientes de sensibilidade associados com as estimativas de entrada $x_{i}$ e $x_{h}$, respectivamente, isto é, as derivadas parciais da relação funcional $f$ com relação às variáveis $X_{i}$ e $X_{h}$, avaliadas nas estimativas $x_{i}$ e $x_{h}$,

$$
c_{i}=\frac{\partial f}{\partial x_{i}}=\left.\frac{\partial f}{\partial X_{i}}\right|_{X_{i}=x_{i}, \cdots, X_{N}=x_{N}},
$$


mesmo raciocínio para $c_{h}$. Temos ainda que $u\left(x_{i}\right)$ é a incerteza padrão associada à estimativa $x_{i}$ da grandeza de entrada $X_{i}$ (avaliada conforme seção anterior) e $u\left(x_{i}, x_{h}\right)$ é a covariância associada às duas estimativas $x_{i}$ e $x_{h}$ dada por

$$
u\left(x_{i}, x_{h}\right)=u\left(x_{i}\right) u\left(x_{h}\right) r\left(x_{i}, x_{h}\right), \quad i \neq h
$$

onde, $r\left(x_{i}, x_{h}\right)$ é o coeficiente de correlação $(i \neq h$ e $|r| \leq 1)$. Em alguns casos, a covariância pode ser estimada por

$$
u\left(x_{i}, x_{h}\right)=\frac{1}{n(n-1)} \sum_{j=1}^{P}\left(x_{i j}-\bar{x}_{i .}\right)\left(x_{h j}-\bar{x}_{h .}\right),
$$

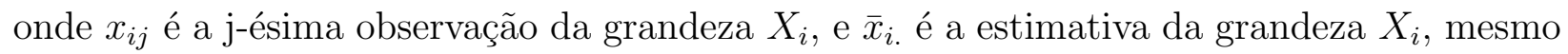
raciocínio para $x_{h j},(i, h=1, \cdots, N)$.

Para grandezas de entrada não correlacionadas, como é o caso do grupo da área de temperatura, a incerteza combinada é dada por :

$$
u_{c}^{2}(y)=\sum_{i=1}^{N} c_{i}^{2} u^{2}\left(x_{i}\right)=\sum_{i=1}^{N}\left(c_{i} u\left(x_{i}\right)\right)^{2}=\sum_{i=1}^{N} u^{2}\left(x_{i}\right) .
$$

O coeficiente de sensibilidade $c_{i}$ descreve o quanto a estimativa de saída $y$ é influenciada por variações da estimativa de entrada $x_{i}$. Como em nossa equação de medição a grandeza de saída é uma função linear das grandezas de entrada, temos que, para todas as grandezas de entrada, $c_{i}=1$.

Logo, utilizando os cálculos das incertezas das grandezas de entrada da seção anterior, obtemos

$$
u_{c}^{2}(y)=(0,0333)^{2}+2 *(0,0288)^{2}+(0,4)^{2}+(0,1)^{2}=0,173056^{\circ} C^{2} .
$$

Assim,

$$
u_{c}(y)=0,416^{\circ} \mathrm{C}
$$

\subsubsection{Incerteza Expandida de Medição}

Embora a incerteza combinada $u_{c}(y)$ possa ser universalmente usada para expressar a incerteza de um resultado de medição, em algumas aplicações comerciais, indústrias e regulamentadoras, é necessário apresentar uma medida de incerteza, que defina um intervalo em torno do resultado da medição. Este intervalo deve abranger uma extensa fração da distribuição de valores que poderiam ser razoavelmente atribuídos ao mensurando.

A medida de incerteza que satisfaz o requisito de fornecer tal intervalo é denominada incerteza expandida e é representada por $u_{e}(y)$. Assim, o resultado de uma medição é representada por

$$
y \pm u_{e}(y) .
$$

A incerteza expandida $u_{e}(y)$ é obtida multiplicando-se a incerteza padrão combinada $u_{c}(y)$ por um fator $\mathrm{k}$ de abrangência, correspondente a uma probabilidade de cobertura especificada (ISO/GUM recomenda 95\%). Logo, a expressão acima pode ser reescrita como sendo

$$
y \pm k * u_{c}(y)
$$


A combinação entre as grandezas de entrada estabelece, pelo Teorema Central do Limite, que a distribuição do mensurando $Y$ será aproximadamente normal. No entanto, podemos ter em alguns casos, que a incerteza padrão combinada pode estar sendo dominada por componentes de incertezas padrão avaliadas pelo Tipo A, baseada em poucas observações, ou por componentes de incertezas padrão avaliadas pelo Tipo B, baseadas em uma suposta distribuição não normal. Com isso, ao invés de usarmos o valor de $k$ da distribuição normal, podemos melhorar a aproximação observando a distribuição da estatística

$$
T=\frac{y-Y}{\sqrt{\hat{\operatorname{Var}}(Y)}}=\frac{y-Y}{\sqrt{u_{c}^{2}(y)}}=\frac{y-Y}{u_{c}(y)},
$$

onde $u_{c}(y)$ é a estimativa para o desvio padrão da grandeza de saída $Y$.

Pelo método de Welch-Satterthwaite, a estatística $T$ tem distribuição aproximadamente t de Student com $\nu_{\text {eff }}$ graus de liberdade. O método utilizado estima os graus de liberdade baseado nos graus de liberdade de cada fonte de incerteza (ISO/GUM, 1998, pg 64), na forma

$$
\nu_{\mathrm{eff}}=\frac{u_{c}^{4}(y)}{\sum_{i=1}^{N} \frac{\left(u_{i}(y)\right)^{4}}{\nu_{i}}},
$$

onde os $u_{i}(y)=c_{i} u_{i}\left(x_{i}\right)$ com $i=1, \cdots, N$, são as contribuições para a incerteza padrão associada à estimativa de saída $y$, resultante da incerteza padrão associada à estimativa de entrada $x_{i}$, e $\nu_{i}$ são os graus de liberdade efetivos da contribuição da incerteza padrão $u_{i}(y)$.

Como a estatística $T$ tem distribuição aproximadamente t de Student, o intervalo com nível de confiança $\gamma$ para o mensurando $Y$ é dado por

$$
P\left(-t_{\frac{\gamma}{2}} \leq T \leq t_{\frac{\gamma}{2}}\right)=(1-\gamma)
$$

ou seja,

$$
-t_{\frac{\gamma}{2}} \leq \frac{y-Y}{u_{c}(y)} \leq t_{\frac{\gamma}{2}} \Rightarrow y \pm t_{\frac{\gamma}{2}} u_{c}(y)
$$

onde $t_{\frac{\gamma}{2}}$ é o quantil $\left(1-\frac{\gamma}{2}\right) * 100 \%$ da distribuição t de Student que depende dos graus de liberdade dados por (2.8). Em geral, se denota $k$ (Fator de Abrangência) ao invés de $t_{\frac{\gamma}{2}}$.

Para os dados da Tabela 2.3, à $25^{\circ} \mathrm{C}$ temos que os graus de liberdade são dados por

$$
\nu_{\mathrm{eff}}=0,416^{4} *\left(\frac{0,033^{4}}{2}+2 * \frac{0,0288^{4}}{30}+\frac{0,4^{4}}{30}+\frac{0,1^{4}}{30}\right)^{-1} \approx 34,820 .
$$

Obs.: Para o cálculo de $\nu_{\text {eff }}$ temos que os graus de liberdade com relação à repetitividade são iguais a 2 (já que foram realizadas 3 medições em cada patamar de temperatura). Para as outras grandezas de entrada da equação de medição foi assumido que todas possuem 30 graus de liberdade, conforme recomendação do INMETRO.

Arredondando para baixo (sempre), temos $\nu_{\text {eff }}=34$. Considerando um nível de confiança de $95 \%$, obtemos da distribuição t de Student que $k \approx 2,032$ e então, a incerteza expandida é dada por

$$
u_{e}(y)=k \cdot u_{c}(y)=2,032 * 0,416=0,845^{\circ} \mathrm{C} .
$$

Resumiremos os cálculos de incerteza apresentados para o laboratório 1 no patamar de $25^{\circ} \mathrm{C}$ em forma de tabela. Para os demais patamares de temperatura para o laboratório 1 e para os outros laboratórios, os cálculos de incerteza em todos os patamares de temperatura para as grandezas de entrada encontram-se no Apêndice B. 
Cálculo de Incerteza: $25^{\circ} \mathrm{C}$

\begin{tabular}{|c|c|c|c|c|c|c|}
\hline Fonte de Incerteza & Estimativa & Tipo & Distribuicão & Divisor & Incerteza & G.L \\
\hline Repetitividade & 0,033 & $\mathrm{~A}$ & Normal & 1 & 0,033333333 & 2 \\
\hline Resolução do Equipamento do lab. & 0,1 & $\mathrm{~B}$ & Retangular & 3,4641 & 0,028867527 & 30 \\
\hline Resolução do Artefato & 0,1 & $\mathrm{~B}$ & Retangular & 3,4641 & 0,028867527 & 30 \\
\hline Incerteza do Indicador (herdada) & 0,8 & $\mathrm{~B}$ & Normal & 2 & 0,4 & 30 \\
\hline Incerteza do Termopar (herdada) & 0,2 & $\mathrm{~B}$ & Normal & 2 & 0,1 & 30 \\
\hline Incerteza Combinada & & & & & & 0,416 \\
\hline Graus de Liberdade Efetivos & & & & & & 34,820 \\
\hline Fator de Abrangência & & & & & & 2,032 \\
\hline Incerteza Expandida & & & & & & 0,845 \\
\hline
\end{tabular}

Tabela 2.5: Cálculo de incertezas para o laboratório 1 a $25^{\circ} \mathrm{C}$ 


\section{Capítulo 3}

\section{Modelo Estatístico, Estimação e Algoritmo EM}

\subsection{Introdução}

Neste capítulo, vamos apresentar um possível modelo para explicar os dados, similar ao proposto por Jaech (1985) e obter aproximações para as estimativas de máxima verossimilhança para o parâmetro de tendência aditiva $\underline{\alpha}$ dos laboratórios e para o parâmetro de tendência multiplicativa $\underline{\beta}$ com respeito ao valor de referência. Aqui, vamos utilizar o algoritmo EM como método numérico para obtenção das aproximações das estimativas de máxima verossimilhança.

\subsection{Modelo Proposto}

Considere um programa de EP com um grupo de N laboratórios, onde cada laboratório realiza uma série de medições de um mesmo artefato. Assim, um possível modelo para explicar os dados é:

$$
Y_{i j k}=\alpha_{i}+\beta_{i} X_{j}+\epsilon_{i j k}, \quad\left\{\begin{array}{l}
j=1, \ldots, P \text { (Patamares de temperatura) } \\
i=1, \ldots, N \text { (Laboratórios) } \\
k=1, \ldots, M \text { (Réplicas) }
\end{array}\right.
$$

onde $Y_{i j k}$ representa a k-ésima tendência do laboratório $i$ no j-ésimo patamar de temperatura, $\alpha_{i}$ representa o parâmetro de tendência aditiva em relação a $X_{j}, \beta_{i}$ é a tendência multiplicativa em relação a $X_{j}$. O erro de medição $\epsilon_{i j k}$ está associado ao laboratório $i$, no patamar de temperatura $j$ e na réplica $k$.

Vamos assumir que $\epsilon_{i j k} \sim N\left(0, \sigma_{i j}^{2}\right), k=1, \ldots M$, onde $\sigma_{i j}$ reflete o desvio padrão do erro do laboratório $i$ associado à sua estimativa de medição $y_{i j k}$, isto é, a incerteza combinada associada à estimativa $y_{i j k}$. Assumimos também, que a variável de comparação $X_{j} \sim N\left(\mu_{j}, \sigma_{x j}^{2}\right)$, onde os seus parâmetros são conhecidos. A variável $X_{j}$ corresponde ao patamar de temperatura j. O parâmetro $\mu_{j}$ corresponde ao patamar de temperatura regulado no forno $\left(25^{\circ} \mathrm{C}, 50^{\circ} \mathrm{C}, 175^{\circ} \mathrm{C}, 300^{\circ} \mathrm{C}\right.$ e $\left.425^{\circ} \mathrm{C}\right)$. Por outro lado, o parâmetro $\sigma_{x j}$ corresponde à incerteza associada ao valor de referência (Tabela 2.1, coluna Desvio Padrão). As variâncias $\sigma_{i j}^{2}$ também são determinadas por calibração pelo respectivo laboratório, conforme apresentado no Capítulo 2. Para simplificar a notação, vamos considerar que cada laboratório realizou o mesmo número $\mathrm{M}$ de réplicas em cada patamar de temperatura.

Desde que cada laboratório tem equipamentos de medição, condições ambientais e operadores distintos, e as réplicas, em cada laboratório, são obtidas em condições de repetitividade, vamos assumir que 
a. $\epsilon_{i j k}$ é independente de $\epsilon_{l j k}$ para todo $k=1, \ldots, M ; i, l=1, \ldots, N, i \neq l ; j=1, \ldots, P$;

b. $\epsilon_{i j k}$ é independente de $\epsilon_{i l k}$ para todo $j, l=1, \ldots, P, j \neq l ; k=1, \ldots, M ; i=1, \ldots, N$;

c. $\epsilon_{i j k}$ é independente de $\epsilon_{i j l}$ para todo $k, l=1, \ldots, M, \quad k \neq l ; i=1, \ldots, N$; $j=1, \ldots, P$

d. $\epsilon_{i j k}$ é independente da variável $X_{j}$, para todo $k=1, \ldots, M ; \quad i=1, \ldots, N$; $j=1, \ldots, P$ e

e. $X_{j}$ é independente de $X_{l}$, para todo $j, l=1, \ldots, P, j \neq l$.

Assim, podemos afirmar que tanto os erros cometidos nas medições do mesmo laboratório quanto os erros nas medições de diferentes laboratórios são independentes. Finalmente, vamos considerar o vetor aleatório normal NM-variado $Y_{j} \sim N\left(\mu_{y j}, V_{y j}\right), j=1, \ldots, P$, onde:

$$
Y_{j}=\left(\begin{array}{c}
Y_{1 j 1} \\
\vdots \\
Y_{1 j M} \\
Y_{2 j 1} \\
\vdots \\
Y_{2 j M} \\
\vdots \\
Y_{N j 1} \\
\vdots \\
Y_{N j M}
\end{array}\right) \quad ; \quad Y=\left(\begin{array}{c}
Y_{1} \\
Y_{2} \\
\vdots \\
Y_{P}
\end{array}\right)
$$

$\mathrm{O}$ vetor $Y_{j}$ corresponde às medições dos laboratórios em cada patamar de temperatura, enquanto que o vetor $\mathrm{Y}$ corresponde a todos os dados.

Sejam

$$
\mu_{y j}=\left(\begin{array}{c}
\alpha_{1}+\beta_{1} \mu_{j} \\
\vdots \\
\alpha_{1}+\beta_{1} \mu_{j} \\
\alpha_{2}+\beta_{2} \mu_{j} \\
\vdots \\
\alpha_{2}+\beta_{2} \mu_{j} \\
\vdots \\
\alpha_{N}+\beta_{N} \mu_{j} \\
\vdots \\
\alpha_{N}+\beta_{N} \mu_{j}
\end{array}\right), \quad \underline{\alpha}=\left(\begin{array}{c}
\alpha_{1} \\
\alpha_{2} \\
\vdots \\
\alpha_{N}
\end{array}\right), \quad \underline{\beta}=\left(\begin{array}{c}
\beta_{1} \\
\beta_{2} \\
\vdots \\
\beta_{N}
\end{array}\right)
$$

o vetor de médias de $Y_{j}$ e os vetores de parâmetros respectivamente e 
$V_{y j}=\left(\begin{array}{ccccccccc}\beta_{1}^{2} \sigma_{x j}^{2}+\sigma_{1 j}^{2} & \beta_{1}^{2} \sigma_{x j}^{2} & \ldots & \beta_{1}^{2} \sigma_{x j}^{2} & \ldots & \beta_{1} \beta_{N} \sigma_{x j}^{2} & \ldots & \beta_{1} \beta_{N} \sigma_{x j}^{2} & \beta_{1} \beta_{N} \sigma_{x j}^{2} \\ \beta_{1}^{2} \sigma_{x j}^{2} & \beta_{1}^{2} \sigma_{x j}^{2}+\sigma_{1 j}^{2} & \ldots & \beta_{1}^{2} \sigma_{x j}^{2} & \ldots & \beta_{1} \beta_{N} \sigma_{x j}^{2} & \ldots & \beta_{1} \beta_{N} \sigma_{x j}^{2} & \beta_{1} \beta_{N} \sigma_{x j}^{2} \\ \vdots & \vdots & \ddots & \vdots & \vdots & \vdots & \vdots & \vdots & \vdots \\ \beta_{1}^{2} \sigma_{x j}^{2} & \beta_{1}^{2} \sigma_{x j}^{2} & \ldots & \beta_{1}^{2} \sigma_{x j}^{2}+\sigma_{1 j}^{2} & \ldots & \beta_{1} \beta_{N} \sigma_{x j}^{2} & \ldots & \beta_{1} \beta_{N} \sigma_{x j}^{2} & \beta_{1} \beta_{N} \sigma_{x j}^{2} \\ \vdots & \vdots & \ldots & \vdots & \ddots & \vdots & \vdots & \vdots & \vdots \\ \beta_{1} \beta_{N} \sigma_{x j}^{2} & \ldots & \beta_{1} \beta_{N} \sigma_{x j}^{2} & \ldots & \beta_{N}^{2} \sigma_{x j}^{2} & \beta_{N}^{2} \sigma_{x j}^{2}+\sigma_{N j}^{2} & \ldots & \beta_{N}^{2} \sigma_{x j}^{2} & \beta_{N}^{2} \sigma_{x j}^{2} \\ \beta_{1} \beta_{N} \sigma_{x j}^{2} & \ldots & \beta_{1} \beta_{N} \sigma_{x j}^{2} & \ldots & \beta_{N}^{2} \sigma_{x j}^{2} & \beta_{N}^{2} \sigma_{x j}^{2} & \beta_{N}^{2} \sigma_{x j}^{2}+\sigma_{N j}^{2} & \ldots & \beta_{N}^{2} \sigma_{x j}^{2} \\ \vdots & \vdots & \vdots & \vdots & \vdots & \vdots & \vdots & \ddots & \vdots \\ \beta_{1} \beta_{N} \sigma_{x j}^{2} & \ldots & \beta_{1} \beta_{N} \sigma_{x j}^{2} & \ldots & \beta_{N}^{2} \sigma_{x j}^{2} & \ldots & \beta_{N}^{2} \sigma_{x j}^{2} & \ldots & \beta_{N}^{2} \sigma_{x j}^{2}+\sigma_{N j}^{2}\end{array}\right)$,

a matriz de covariâncias de $Y_{j}$.

Desta forma, podemos escrever $V_{y j}=\underline{\beta}^{*} \underline{\beta}^{*^{\prime}} \sigma_{x j}^{2}+D_{j}$, onde,

$$
\underline{\beta}^{*}=\left(\begin{array}{llllllllll}
\beta_{1} & \ldots & \beta_{1} & \beta_{2} & \ldots & \beta_{2} & \ldots & \beta_{N} & \ldots & \beta_{N}
\end{array}\right)^{\prime}
$$

e $D_{j}$ é a matriz diagonal com elementos

$$
\left(\begin{array}{llllllll}
\sigma_{1 j}^{2}, & \ldots, & \sigma_{1 j}^{2}, & \sigma_{2 j}^{2}, & \ldots, & \sigma_{2 j}^{2}, & \ldots, & \sigma_{N j}^{2},
\end{array}, \sigma_{N j}^{2}\right), j=1, \ldots P .
$$

Desse modo, $Y_{i j k} \sim N\left(\alpha_{i}+\beta_{i} \mu_{j}, \beta_{i}^{2} \sigma_{x j}^{2}+\sigma_{i j}^{2}\right)$ (Srivastava \& Khatri, 1979, Teorema 2.2.2, pg 44). O determinante de $V_{y j}$ é dado por (Graybill, 1983, Teorema 8.4.3, pg 203)

$$
\left|V_{y j}\right|=\left[1+\sigma_{x j}^{2}\left(\frac{\beta_{1}^{2}}{\sigma_{1 j}^{2}}+\ldots+\frac{\beta_{1}^{2}}{\sigma_{1 j}^{2}}+\ldots+\frac{\beta_{N}^{2}}{\sigma_{N j}^{2}}+\ldots+\frac{\beta_{N}^{2}}{\sigma_{N j}^{2}}\right)\right] \sigma_{1 j}^{2} \ldots \sigma_{1 j}^{2} \ldots \sigma_{N j}^{2} \ldots \sigma_{N j}^{2}
$$

e a inversa $V_{y j}{ }^{-1}$ é dada por (Graybill, 1983, Teorema 8.3.3, pg 189)

$$
V_{y j}^{-1}=D_{j}^{-1}-\frac{\sigma_{x j}^{2}\left(D_{j}^{-1} \underline{\beta}^{*}\right)\left(\underline{\beta}^{*^{\prime}} D_{j}^{-1}\right)}{1+\sigma_{x j}^{2} \underline{\beta}^{*^{\prime}} D_{j}^{-1} \underline{\beta}^{*}}, j=1, \ldots P .
$$

Logo, a função de verossimilhança do modelo proposto é dada por

$$
f_{y}=\prod_{j=1}^{P} f_{y j}=(2 \pi)^{-\frac{N M P}{2}} \prod_{j=1}^{P}\left|V_{y j}\right|^{-\frac{1}{2}} \exp \left[-\frac{1}{2} \sum_{j=1}^{P}\left(y_{j}-\mu_{y j}\right)^{\prime} V_{y j}{ }^{-1}\left(y_{j}-\mu_{y j}\right)\right]
$$

\subsection{Introdução da Variável Latente e Algoritmo EM}

Os cálculos necessários para se obter os estimadores de máxima verossimilhança dos parâmetros $\underline{\alpha}$ e $\beta$ são complicados, pois o determinante de $V_{y j}$ depende do vetor de parâmetros $\beta$. Desse modo, vamos incorporar a variável explicativa $X_{j}$ no vetor $Y_{j}$ e obter uma nova função de verossimilhança, onde o determinante da matriz de covariâncias não dependerá de $\beta$. Aqui, pretendemos obter aproximações das estimativas de máxima verossimilhança para os parâmetros $\underline{\alpha}$ e $\underline{\beta}$ através de procedimentos iterativos, como o algoritmo EM (ver, Dempster, Laird e Rubin, 1977). Seja o vetor de "dados" aumentados 


$$
Y_{j}^{*}=\left(\begin{array}{c}
X_{j} \\
Y_{1 j 1} \\
\vdots \\
Y_{1 j M} \\
Y_{2 j 1} \\
\vdots \\
Y_{2 j M} \\
\vdots \\
Y_{N j 1} \\
\vdots \\
Y_{N j M}
\end{array}\right), \quad Y^{*}=\left(\begin{array}{c}
Y_{1}^{*} \\
Y_{2}^{*} \\
\vdots \\
Y_{P}^{*}
\end{array}\right)
$$

Admitimos que $Y_{j}^{*} \sim N_{N M+1}\left(\mu_{y j}^{*}, V_{y j}^{*}\right), j=1, \ldots, P$, onde

$$
\mu_{y j}^{*}=\left(\begin{array}{c}
\mu_{j} \\
\alpha_{1}+\beta_{1} \mu_{j} \\
\vdots \\
\alpha_{1}+\beta_{1} \mu_{j} \\
\alpha_{2}+\beta_{2} \mu_{j} \\
\vdots \\
\alpha_{2}+\beta_{2} \mu_{j} \\
\vdots \\
\alpha_{N}+\beta_{N} \mu_{j} \\
\vdots \\
\alpha_{N}+\beta_{N} \mu_{j}
\end{array}\right)
$$

e a matriz $V_{y j}^{*}$, na forma particionada é dada por

$$
V_{y j}^{*}=\left(\begin{array}{cc}
\sigma_{x j}^{2} & \underline{\beta}^{*^{\prime}} \sigma_{x j}^{2} \\
\underline{\beta}^{*} \sigma_{x j}^{2} & \Sigma_{y j}^{*}
\end{array}\right) .
$$

O determinante de $V_{y j}^{*}$ é dado por (Graybill, 1983, Teorema 8.2.1, item 3, pg 184)

$\left|V_{y j}^{*}\right|=\left|\sigma_{x j}^{2}\right|\left|\underline{\beta}^{*} \underline{\beta}^{*^{\prime}} \sigma_{x j}^{2}+D_{j}-\underline{\beta}^{*} \frac{1}{\sigma_{x j}^{2}} \underline{\beta}^{*^{\prime}} \sigma_{x j}^{2}\right|=\sigma_{x j}^{2}\left|D_{j}\right|=\sigma_{x j}^{2} \sigma_{1 j}^{2} \ldots \sigma_{1 j}^{2} \ldots \sigma_{N j}^{2} \ldots \sigma_{N j}^{2}$.

Para calcularmos a inversa de $V_{y j}^{*}$, tomamos

$$
V_{y j}^{*-1}=\left(\begin{array}{ll}
W_{11} & W_{12} \\
W_{21} & W_{22}
\end{array}\right)
$$

onde (Graybill, 1983, Teorema 8.2.1 item 1, pg 184)

$$
W_{11}=\left[\sigma_{x j}^{2}-\underline{\beta}^{*^{\prime}} \sigma_{x j}^{2} \Sigma_{y j}^{*-1} \underline{\beta}^{*} \sigma_{x j}^{2}\right]^{-1} .
$$

A inversa da matriz $\Sigma_{y j}^{*}$ é dada por (Graybill, 1983, Teorema 8.3.3, pg 189)

$\Sigma_{y j}^{*-1}=D_{j}^{-1}-\frac{\left(D_{j}^{-1} \underline{\beta}^{*}\right)\left(\underline{\beta}^{*^{\prime}} D_{j}^{-1}\right) \sigma_{x j}^{2}}{1+\underline{\beta}^{*^{\prime}} D_{j}^{-1} \underline{\beta}^{*} \sigma_{x j}^{2}}$.

Então, $W_{11}$ é dado por 
$W_{11}=\left[\sigma_{x j}^{2}-\underline{\beta}^{*^{\prime}} \sigma_{x j}^{2}\left(D_{j}^{-1}-\frac{\left(D_{j}^{-1} \underline{\beta}^{*}\right)\left(\underline{\beta}^{*^{\prime}} D_{j}^{-1}\right) \sigma_{x j}^{2}}{1+\underline{\beta}^{*^{\prime}} D_{j}^{-1} \underline{\beta}^{*} \sigma_{x j}^{2}}\right) \underline{\beta}^{*} \sigma_{x j}^{2}\right]^{-1}$.

Através de manipulações algébricas, encontramos

$$
\begin{aligned}
& W_{11}=\frac{1}{\sigma_{x j}^{2}}+\underline{\beta}^{*^{\prime}} D_{j}^{-1} \underline{\beta}^{*} \\
& W_{12}=-\frac{1}{\sigma_{x j}^{2}} \underline{\beta}^{*^{\prime}} \sigma_{x j}^{2}\left[\underline{\beta^{*}} \underline{\beta}^{*^{\prime}} \sigma_{x j}^{2}+D_{j}-\underline{\beta}^{*} \sigma_{x j}^{2} \frac{1}{\sigma_{x j}^{2}} \underline{\beta}^{*^{\prime}} \sigma_{x j}^{2}\right]^{-1}=-\underline{\beta}^{*^{\prime}} D_{j}^{-1} \\
& W_{21}=W_{12}^{\prime}=-D_{j}^{-1} \underline{\beta^{*}} ; \\
& W_{22}=\left[\underline{\beta}^{*} \underline{\beta^{*^{\prime}}} \sigma_{x j}^{2}+D_{j}-\underline{\beta}^{*} \underline{\beta^{*^{\prime}}} \sigma_{x j}^{2}\right]^{-1}=D_{j}^{-1} .
\end{aligned}
$$

Para o modelo aumentado, a função de verossimilhança é dada por

$$
f_{y^{*}}=\prod_{j=1}^{P} f_{y_{j}^{*}}=(2 \pi)^{-\frac{(N M+1) P}{2}} \prod_{j=1}^{P}\left|V_{y j}^{*}\right|^{-\frac{1}{2}} \exp \left[-\frac{1}{2} \sum_{j=1}^{P}\left(y_{j}^{*}-\mu_{y j}^{*}\right)^{\prime} V_{y j}^{*-1}\left(y_{j}^{*}-\mu_{y j}^{*}\right)\right]
$$

A expressão no termo exponencial é dada por

$$
\left(y_{j}^{*}-\mu_{y j}^{*}\right)^{\prime} V_{y j}^{*-1}\left(y_{j}^{*}-\mu_{y j}^{*}\right)=y_{j}^{*^{\prime}} V_{y j}^{*-1} y_{j}^{*}-2 \mu_{y j}^{*^{\prime}} V_{y j}^{*-1} y_{j}^{*}+\mu_{y j}^{*^{\prime}} V_{y j}^{*-1} \mu_{y j}^{*}
$$

e

$$
\left(y_{j}^{*}-\mu_{y j}^{*}\right)^{\prime} V_{y j}^{*-1}\left(y_{j}^{*}-\mu_{y j}^{*}\right)=\left(\begin{array}{c}
x_{j}-\mu_{j} \\
\underline{y_{j}}-\left(\underline{\alpha^{*}}+\mu_{j} \underline{\beta^{*}}\right)
\end{array}\right)^{\prime}\left(\begin{array}{ll}
W_{11} & W_{12} \\
W_{21} & W_{22}
\end{array}\right)\left(\begin{array}{c}
x_{j}-\mu_{j} \\
\underline{y_{j}}-\left(\underline{\alpha^{*}}+\mu_{j} \underline{\beta^{*}}\right)
\end{array}\right)
$$

onde

$$
\begin{gathered}
\underline{\alpha^{*}}=\left(\begin{array}{llllllllll}
\alpha_{1} & \ldots & \alpha_{1} & \alpha_{2} & \ldots & \alpha_{2} & \ldots & \alpha_{N} & \ldots & \alpha_{N}
\end{array}\right)^{\prime}, \\
\underline{y_{j}}=\left(\begin{array}{llllllllll}
y_{1 j 1} & \ldots & y_{1 j M} & y_{2 j 1} & \ldots & y_{2 j M} & \ldots & y_{N j 1} & \ldots & y_{N j M}
\end{array}\right)^{\prime} .
\end{gathered}
$$

Das equações (3.4) e (3.5), obtemos

$$
\begin{aligned}
& \left(y_{j}^{*}-\mu_{y j}^{*}\right)^{\prime} V_{y j}^{*-1}\left(y_{j}^{*}-\mu_{y j}^{*}\right)=\left(\begin{array}{ll}
x_{j} & \underline{y_{j}^{\prime}}
\end{array}\right)\left(\begin{array}{ll}
W_{11} & W_{12} \\
W_{21} & W_{22}
\end{array}\right)\left(\begin{array}{l}
x_{j} \\
\underline{y_{j}^{\prime}}
\end{array}\right)+
\end{aligned}
$$

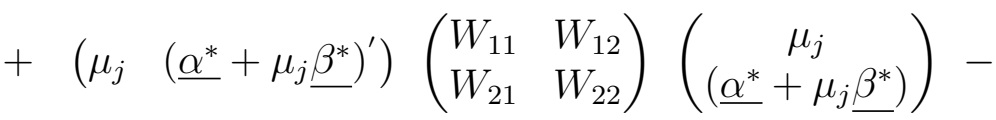

$$
\begin{aligned}
& -2\left(\mu_{j}\left(\underline{\alpha^{*}}+\mu_{j} \underline{\beta^{*^{\prime}}}\right)\right)\left(\begin{array}{ll}
W_{11} & W_{12} \\
W_{21} & W_{22}
\end{array}\right)\left(\begin{array}{l}
x_{j} \\
\underline{y_{j}^{\prime}}
\end{array}\right) \text {. }
\end{aligned}
$$

Portanto, 


$$
\begin{aligned}
\left(y_{j}^{*}-\mu_{y j}^{*}\right)^{\prime} V_{y j}^{*-1}\left(y_{j}^{*}-\mu_{y j}^{*}\right) & =x_{j}^{2} W_{11}+x_{j} \underline{y_{j}^{\prime}} W_{21}+x_{j} W_{12} \underline{y_{j}}+\underline{y_{j}^{\prime}} W_{22} \underline{y_{j}}+\mu_{j}^{2} W_{11} \\
& +\mu_{j}\left(\underline{\alpha^{*}}+\mu_{j} \underline{\left.\beta^{*}\right)^{\prime}} W_{21}+\mu_{j} W_{12} \underline{\left(\alpha^{*}\right.}+\mu_{j} \underline{\beta^{*}}\right) \\
& +\left(\underline{\alpha^{*}}+\mu_{j} \underline{\left.\beta^{*}\right)^{\prime}} W_{22} \underline{\left(\alpha^{*}\right.}+\mu_{j} \underline{\beta^{*}}\right)-2 \mu_{j} x_{j} W_{11} \\
& -2 x_{j} \underline{\left(\alpha^{*}+\mu_{j}\right.} \underline{\left.\beta^{*}\right)^{\prime}} W_{21}-2 \mu_{j} W_{12} \underline{y_{j}^{\prime}} \\
& -2\left(\underline{\alpha^{*}}+\mu_{j} \underline{\beta^{*}}\right)^{\prime} W_{22} \underline{y_{j}} .
\end{aligned}
$$

Substituindo os valores de $W_{11}, W_{12}, W_{21}$ e $W_{22}$, obtemos que

$$
\begin{aligned}
\left(y_{j}^{*}-\mu_{y j}^{*}\right)^{\prime} V_{y j}^{*-1}\left(y_{j}^{*}-\mu_{y j}^{*}\right) & =\frac{\left(x_{j}-\mu_{j}\right)^{2}}{\sigma_{x j}^{2}}+x_{j}^{2} \underline{\beta}^{*^{\prime}} D_{j}^{-1} \underline{\beta}^{*}+ \\
& +2 x_{j}\left(\underline{\alpha}^{*^{\prime}}-\underline{y}_{j}^{\prime}\right) D_{j}^{-1} \underline{\beta}^{*}+\underline{y j}_{j}^{\prime} D_{j}^{-1} \underline{y_{j}}+ \\
& +\underline{\alpha}^{*^{\prime}} D_{j}^{-1} \underline{\alpha}^{*}-2 \underline{\alpha}^{*^{\prime}} D_{j}^{-1} \underline{y_{j}} .
\end{aligned}
$$

Logo, a função log-verossimilhança para o modelo aumentado é dada por

$$
\begin{aligned}
L\left(\underline{\alpha}, \underline{\beta} / Y^{*}\right) & =\operatorname{Ln}\left[\prod_{j=1}^{P} f_{y_{j}^{*}}\right]=-\frac{(N M+1) P}{2} \ln (2 \pi)-\frac{1}{2} \sum_{j=1}^{P} \ln \left(\sigma_{x j}^{2} \sigma_{1 j}^{2} \ldots \sigma_{1 j}^{2} \ldots \sigma_{N j}^{2} \ldots \sigma_{N j}^{2}\right)- \\
& -\frac{1}{2}\left[\sum _ { j = 1 } ^ { P } \left(\frac{\left(x_{j}-\mu_{j}\right)^{2}}{\sigma_{x j}^{2}}+x_{j}^{2} \underline{\beta}^{*^{\prime}} D_{j}^{-1} \underline{\beta}^{*}+2 x_{j}\left(\underline{\alpha}^{*^{\prime}}-\underline{y}_{j}^{\prime}\right) D_{j}^{-1} \underline{\beta}^{*}+\right.\right. \\
& \left.\left.+\underline{y_{j}^{\prime}} D_{j}^{-1} \underline{y_{j}}+\underline{\alpha}^{*^{\prime}} D_{j}^{-1} \underline{\alpha}^{*}-2 \underline{\alpha}^{*^{\prime}} D_{j}^{-1} \underline{y_{j}}\right)\right] .
\end{aligned}
$$

Como para o nosso modelo os parâmetros são $\underline{\alpha}$ e $\underline{\beta}$, vamos escrever $\underline{\beta}^{*}=J \underline{\beta}$ e $\underline{\alpha}^{*}=J \underline{\alpha}$, onde a matriz J é dada por

$$
J=\left(\begin{array}{ccccc}
1 & 0 & 0 & \ldots & 0 \\
\vdots & \vdots & \vdots & \vdots & \vdots \\
1 & 0 & 0 & \ldots & 0 \\
0 & 1 & 0 & \ldots & 0 \\
\vdots & \vdots & \vdots & \vdots & \vdots \\
0 & 1 & 0 & \ldots & 0 \\
\vdots & \vdots & \vdots & \vdots & \vdots \\
0 & 0 & 0 & \ldots & 1 \\
\vdots & \vdots & \vdots & \vdots & \vdots \\
0 & 0 & 0 & \ldots & 1
\end{array}\right)
$$

Desta forma, podemos reescrever o log da função de verossimilhança do modelo aumentado como 


$$
\begin{aligned}
L\left(\underline{\alpha}, \underline{\beta} / Y^{*}\right) & =\operatorname{Ln}\left[\prod_{j=1}^{P} f_{y_{j}^{*}}\right]=-\frac{(N M+1) P}{2} \ln (2 \pi)-\frac{1}{2} \sum_{j=1}^{P} \ln \left(\sigma_{x j}^{2} \sigma_{1 j}^{2} \ldots \sigma_{1 j}^{2} \ldots \sigma_{N j}^{2} \ldots \sigma_{N j}^{2}\right)- \\
& -\frac{1}{2}\left[\sum _ { j = 1 } ^ { P } \left(\frac{\left(x_{j}-\mu_{j}\right)^{2}}{\sigma_{x j}^{2}}+x_{j}^{2}(J \underline{\beta})^{\prime} D_{j}^{-1} J \underline{\beta}+2 x_{j}\left((J \underline{\alpha})^{\prime}-\underline{y}_{j}^{\prime}\right) D_{j}^{-1} J \underline{\beta}+\right.\right. \\
& \left.\left.+\underline{y_{j}^{\prime}} D_{j}^{-1} \underline{y_{j}}+(J \underline{\alpha})^{\prime} D_{j}^{-1} J \underline{\alpha}-2(J \underline{\alpha})^{\prime} D_{j}^{-1} \underline{y_{j}}\right)\right] .
\end{aligned}
$$

Na próxima seção, apresentamos o algoritmo EM e condições suficientes para a sua convergência. Aqui, apenas aplicamos os conceitos das etapas E e M para o nosso modelo.

\subsubsection{Etapa E}

Aqui, obtemos a esperança da função log- verossimilhança dos dados aumentados (equação 3.7) dadas as observações y e os parâmetros estimados $(\underline{\alpha}, \beta)$. Para implementar esta etapa, é suficiente obter a esperança condicional das estatísticas suficientes da distribuição do vetor $Y^{*}=\left(\begin{array}{llll}Y_{1}^{*} & Y_{2}^{*} & \cdots & Y_{P}^{*}\end{array}\right)^{\prime}$ dado os dados observados $Y=\left(\begin{array}{lllll}Y_{1} & Y_{2} & \cdots & Y_{P}\end{array}\right)^{\prime}$ e os parâmetros $(\underline{\alpha}, \underline{\beta})$, pois o log da função de verossimilhança é obtido de uma distribuição da família exponencial regular (Dempster, Laird \& Rubin, 1977). Tomamos o vetor particionado $\mu_{y j}^{*}=\left(\begin{array}{c}\mu_{j} \\ \mu_{y j}\end{array}\right)$ e a matriz $V_{y j}^{*}$ da forma particionada. Então (Searle, 1971, pps. 46, 47),

$$
\left.\hat{x_{j}}=E\left(X_{j} / Y_{j}=\underline{y_{j}}\right)=\mu_{j}+\underline{\beta}^{*^{\prime}} \sigma_{x j}^{2}\left[D_{j}^{-1}-\frac{\left(D_{j}^{-1} \underline{\beta}^{*}\right)\left(\underline{\beta}^{*^{\prime}} D_{j}^{-1}\right) \sigma_{x j}^{2}}{1+\underline{\beta}^{*^{\prime}} D_{j}^{-1} \underline{\beta}^{*} \sigma_{x j}^{2}}\right] \underline{\left(y_{j}\right.}-\mu_{y j}\right),
$$

$\mathrm{e}$

$$
\operatorname{Var}\left(X_{j} / Y_{j}=\underline{y_{j}}\right)=\sigma_{x j}^{2}-\underline{\beta}^{*^{\prime}} \sigma_{x j}^{4}\left[D_{j}^{-1}-\frac{\left(D_{j}^{-1} \underline{\beta}^{*}\right)\left(\underline{\beta}^{*^{\prime}} D_{j}^{-1}\right) \sigma_{x j}^{2}}{1+\underline{\beta}^{*^{\prime}} D_{j}^{-1} \underline{\beta}^{*} \sigma_{x j}^{2}}\right] \underline{\beta}^{*} .
$$

Como $\operatorname{Var}\left(X_{j} / Y_{j}=\underline{y_{j}}\right)=E\left(X_{j}^{2} / Y_{j}=\underline{y_{j}}\right)-\left[E\left(X_{j} / Y_{j}=\underline{y_{j}}\right)\right]^{2}$, temos então que

$$
\begin{aligned}
\hat{x}_{j}^{2}=E\left(X_{j}^{2} / Y_{j}=\underline{y_{j}}\right) & =\sigma_{x j}^{2}-\underline{\beta}^{*^{\prime}} \sigma_{x j}^{4}\left[D_{j}^{-1}-\frac{\left(D_{j}^{-1} \underline{\beta}^{*}\right)\left(\underline{\beta}^{*^{\prime}} D_{j}^{-1}\right) \sigma_{x j}^{2}}{1+\underline{\beta}^{*^{\prime}} D_{j}^{-1} \underline{\beta}^{*} \sigma_{x j}^{2}}\right] \underline{\beta}^{*}+ \\
& \left.+\left[\mu_{j}+\underline{\beta}^{*^{\prime}} \sigma_{x j}^{2}\left[D_{j}^{-1}-\frac{\left(D_{j}^{-1} \underline{\beta}^{*}\right)\left(\underline{\beta}^{*^{\prime}} D_{j}^{-1}\right) \sigma_{x j}^{2}}{1+\underline{\beta}^{*^{\prime}} D_{j}^{-1} \underline{\beta}^{*} \sigma_{x j}^{2}}\right] \underline{\left(y_{j}\right.}-\mu_{y j}\right)\right]^{2},
\end{aligned}
$$

$j=1, \ldots, P$.

\subsubsection{Etapa $\mathrm{M}$}

A etapa M é implementada maximizando o log da função de verossimilhança (equação 3.7). Derivando esta equação em relação aos parâmetros e igualando a zero temos

$$
\frac{\partial}{\partial \underline{\beta}}\left[-\frac{1}{2} \sum_{j=1}^{P}\left(x_{j}^{2} \underline{\beta^{\prime}} J^{\prime} D_{j}^{-1} J \underline{\beta}+2 x_{j}\left(\underline{\alpha^{\prime}} J^{\prime}-\underline{y_{j}^{\prime}}\right) D_{j}^{-1} J \underline{\beta}\right)\right]=0 \text {. }
$$


Logo,

$$
\sum_{j=1}^{P} x_{j}^{2} J^{\prime} D_{j}^{-1} J \underline{\hat{\beta}}+\sum_{j=1}^{P} x_{j} J^{\prime} D_{j}^{-1}\left(J \underline{\hat{\alpha}}-\underline{y_{j}}\right)=0 .
$$

Portanto,

$$
\underline{\hat{\beta}}=-\left(\sum_{j=1}^{P} x_{j}^{2} J^{\prime} D_{j}^{-1} J\right)^{-1}\left[\sum_{j=1}^{P} x_{j} J^{\prime} D_{j}^{-1}\left(J \underline{\hat{\alpha}}-\underline{y_{j}}\right)\right] .
$$

Fazendo $\frac{\partial L\left(\underline{\alpha}, \underline{\beta} / Y^{*}\right)}{\partial \underline{\alpha}}=0$, temos

$$
\frac{\partial}{\partial \underline{\alpha}}\left[-\frac{1}{2} \sum_{j=1}^{P}\left(2 x_{j} \underline{\beta^{\prime}} J^{\prime} D_{j}^{-1}\left(J \underline{\alpha}-\underline{y_{j}}\right)+\underline{\alpha^{\prime}} J^{\prime} D_{j}^{-1} J \underline{\alpha}-2 \underline{y_{j}^{\prime}} D_{j}^{-1} J \underline{\alpha}\right)\right]=0 .
$$

Logo,

$$
\sum_{j=1}^{P} x_{j} J^{\prime} D_{j}^{-1} J \underline{\hat{\beta}}+\sum_{j=1}^{P} J^{\prime} D_{j}^{-1} J \underline{\hat{\alpha}}-\sum_{j=1}^{P} J^{\prime} D_{j}^{-1} \underline{y_{j}}=0
$$

Portanto,

$$
\underline{\hat{\alpha}}=\left[\sum_{j=1}^{P} J^{\prime} D_{j}^{-1} J\right]^{-1}\left(\sum_{j=1}^{P} J^{\prime} D_{j}^{-1} \underline{y_{j}}-\sum_{j=1}^{P} x_{j} J^{\prime} D_{j}^{-1} J \underline{\hat{\beta}}\right) .
$$

Agora, substituindo $\underline{\hat{\alpha}}$ da expressão (3.15) na expressão (3.12) e com algumas manipulações algébricas, encontramos

$$
\begin{aligned}
\underline{\hat{\beta}} & =\left[I_{N}-\left(\sum_{j=1}^{P} x_{j}^{2} J^{\prime} D_{j}^{-1} J\right)^{-1} \sum_{j=1}^{P} x_{j} J^{\prime} D_{j}^{-1} J\left(\sum_{j=1}^{P} J^{\prime} D_{j}^{-1} J\right)^{-1}\left(\sum_{j=1}^{P} x_{j} J^{\prime} D_{j}^{-1} J\right)\right]^{-1} \times \\
& \times\left[\left(\sum_{j=1}^{P} x_{j}^{2} J^{\prime} D_{j}^{-1} J\right)^{-1} \sum_{j=1}^{P} x_{j} J^{\prime} D_{j}^{-1} \underline{y_{j}}-\left(\sum_{j=1}^{P} x_{j}^{2} J^{\prime} D_{j}^{-1} J\right)^{-1}\left(\sum_{j=1}^{P} x_{j} J^{\prime} D_{j}^{-1} J\right) \times\right. \\
& \left.\times\left(\sum_{j=1}^{P} J^{\prime} D_{j}^{-1} J\right)^{-1} \sum_{j=1}^{P} J^{\prime} D_{j}^{-1} \underline{y_{j}}\right] .
\end{aligned}
$$

onde, $I_{N}$ é a matriz identidade de ordem N. Finalmente, substituindo a expressão de $\hat{\beta}$ obtida em (3.16) na equação (3.15), temos 


$$
\begin{aligned}
\underline{\hat{\alpha}} & =\left[\sum_{j=1}^{P} J^{\prime} D_{j}^{-1} J\right]^{-1}\left\{\sum_{j=1}^{P} J^{\prime} D_{j}^{-1} \underline{y}_{j}-\left(\sum_{j=1}^{P} x_{j} J^{\prime} D_{j}^{-1} J\right)\left[I_{N}-\left(\sum_{j=1}^{P} x_{j}^{2} J^{\prime} D_{j}^{-1} J\right)^{-1} \times\right.\right. \\
& \left.\times \sum_{j=1}^{P} x_{j} J^{\prime} D_{j}^{-1} J\left(\sum_{j=1}^{P} J^{\prime} D_{j}^{-1} J\right)^{-1}\left(\sum_{j=1}^{P} x_{j} J^{\prime} D_{j}^{-1} J\right)\right]\left[\left(\sum_{j=1}^{P} x_{j}^{2} J^{\prime} D_{j}^{-1} J\right)^{-1} \times\right. \\
& \times \sum_{j=1}^{P} x_{j} J^{\prime} D_{j}^{-1} \underline{y_{j}}-\left(\sum_{j=1}^{P} x_{j}^{2} J^{\prime} D_{j}^{-1} J\right)^{-1}\left(\sum_{j=1}^{P} x_{j} J^{\prime} D_{j}^{-1} J\right)\left(\sum_{j=1}^{P} J^{\prime} D_{j}^{-1} J\right)^{-1} \times \\
& \left.\left.\times \sum_{j=1}^{P} J^{\prime} D_{j}^{-1} \underline{y_{j}}\right]\right\} .
\end{aligned}
$$

\subsubsection{Algoritmo EM}

Para encontrarmos as estimativas de máxima verossimilhança do modelo, procedemos da seguinte forma:

a. Fornecer um valor inicial para os vetores de parâmetros $\underline{\alpha}$ e $\underline{\beta}$;

b. Obter $\hat{x}_{j}$ pela equação (3.8) e $\hat{x}_{j}^{2}$ pela equação (3.9);

c. Substituir os valores encontrados em (b) nas expressões de $\underline{\hat{\alpha}}(3.17)$ e $\underline{\hat{\beta}}$ (3.16);

d. Substituir os novos valores de $\underline{\hat{\alpha}}$ e $\hat{\beta}$ e voltar ao item (b) até que as diferenças obtidas de uma iteração para outra sejam menores que um valor pré fixado.

\subsection{Estudo da Convergência do Algoritmo EM}

O algoritmo Esperança e Maximização (EM) é um método computacional iterativo para se obter, entre outras aplicações, aproximações para as estimativas de máxima verossimilhança em problemas com dados incompletos, onde a aplicação de outros métodos computacionais tal como o método de Newton - Raphson pode ser mais complicada.

Em cada iteração do algoritmo EM existem dois passos - chamados de passo esperança ou passo E e passo maximização ou passo M. Este nome foi dado por Dempster, Laird e Rubin (1977). O algoritmo EM fornece uma estimativa para os dados que estão faltando, onde os parâmetros são estimados após ser fornecido um valor inicial para estes dados e então reestimados procedendo iterativamente até a convergência. Nesta seção, vamos apresentar o algoritmo EM e estabelecer condições suficientes para sua convergência. Na seqüência, aplicamos o resultado de convergência para o nosso modelo.

\subsubsection{Formulação do Algoritmo EM}

Seja Y o vetor aleatório correspondente aos dados observados y, tendo função densidade de probabilidade $g_{y}(y, \Psi)$, onde $\Psi$ é o vetor de parâmetros desconhecidos. O espaço paramétrico será denotado por $\Omega$ e corresponde a um subconjunto aberto do $\mathbb{R}^{N}$.

Considere $x$ o vetor de dados não observados, $y^{*}=(x, y)$ o vetor de dados completos $\mathrm{e}$ $\prod_{j=1}^{P} f_{y_{j}^{*}}$ a função de verossimilhança deste vetor. Então o log da função de verossimilhança dos dados completos é dada por

$$
L_{c}(\Psi)=\operatorname{Ln} \prod_{j=1}^{P} f_{y_{j}^{*}}
$$


O algoritmo EM aplica-se ao problema de resolver a equação de máxima verossimilhança de dados incompletos

$$
\frac{\partial L(\Psi)}{\partial \Psi}=0
$$

onde $L$ é o log da função de verossimilhança de dados incompletos, indiretamente pelo processo iterativo em termos do log da função de verossimilhança dos dados completos $L_{c}(\Psi)$. Como a função é não observável, ela é substituída pela esperança condicional de $\mathrm{X}$ dado $y^{*}$, utilizando a função ajustada por $\Psi$.

Mais especificamente, seja $\Psi^{(0)}$ algum valor inicial para $\Psi$. Então, na primeira iteração, o passo E requer o cálculo de

$$
Q\left(\Psi, \Psi^{(0)}\right)=E_{\Psi^{(0)}}\left\{L_{c}(\Psi) / y\right\}
$$

O passo M requer a maximização de $Q\left(\Psi, \Psi^{(0)}\right)$ com respeito a $\Psi$ sobre o espaço paramétrico $\Omega$. Isto é, escolhemos $\Psi^{(1)}$ tal que

$$
Q\left(\Psi^{(1)}, \Psi^{(0)}\right) \geq Q\left(\Psi, \Psi^{(0)}\right)
$$

para todo $\Psi \in \Omega$. Os passos E e M são então iterados novamente, porém com $\Psi^{(0)}$ substituído pelo parâmetro ajustado $\Psi^{(1)}$. $\mathrm{Na}(k+1)$-ésima iteração, os passos E e M são definidos como segue (McLachlan e Krishnan, 1997, pp. 21, 22 e 23) :

- Passo E : Calcular $Q\left(\Psi, \Psi^{(k)}\right)$, onde $Q\left(\Psi, \Psi^{(k)}\right)=E_{\Psi^{(k)}}\left\{L_{c}(\Psi) / y\right\}$;

- Passo M : Escolher $\Psi^{(k+1)}$ como sendo um valor de $\Psi \in \Omega$ que maximiza $Q\left(\Psi, \Psi^{(k)}\right)$, isto é,

$$
Q\left(\Psi^{(k+1)}, \Psi^{(k)}\right) \geq Q\left(\Psi, \Psi^{(k)}\right)
$$

para todo $\Psi \in \Omega$.

Os passos E e M são alternadamente repetidos até que a diferença

$$
L\left(\Psi^{(k+1)}\right)-L\left(\Psi^{(k)}\right)
$$

seja uma quantidade arbitrariamente pequena no caso da convergência da sequência de valores de verossimilhança $\left\{L_{c}\left(\Psi^{(k)}\right)\right\}$. Dempster, Laird e Rubin (1977), mostram que a função de verossimilhança (dados incompletos), não decresce após uma iteração EM, isto implica que $L(\Psi)$ também não decresce, ou seja,

$$
L\left(\Psi^{(k+1)}\right) \geq L\left(\Psi^{(k)}\right)
$$

para $k=0,1,2, \ldots$ (ver Dempster, Laird e Rubin, 1977, Teorema 1).

\subsubsection{Passo M Aplicado ao Nosso Modelo}

Como descrito na seção 3.3.2, maximizamos diretamente o log da função de verossimilhança de dados completos $L_{c}(\Psi)$. Porém, o passo M requer que maximizemos $E_{\Psi^{(k)}}\left\{L_{c}(\Psi) / y\right\}$. A seguir, vamos mostrar que estes dois procedimentos de maximização são equivalentes. 


$$
\begin{aligned}
E\left[L_{c}(\Psi) / \Psi^{(0)}, Y^{*}\right] & =E\left[-\frac{(N M+1) P}{2} \ln (2 \pi)-\frac{1}{2} \sum_{j=1}^{P} \ln \left(\sigma_{x j}^{2} \sigma_{1 j}^{2} \ldots \sigma_{1 j}^{2} \ldots \sigma_{N j}^{2} \ldots \sigma_{N j}^{2}\right)-\right. \\
& -\frac{1}{2}\left[\sum _ { j = 1 } ^ { P } \left(\frac{\left(x_{j}-\mu_{j}\right)^{2}}{\sigma_{x j}^{2}}+x_{j}^{2} \underline{\beta}^{*^{\prime}} D_{j}^{-1} \underline{\beta}^{*}+2 x_{j}\left(\underline{\alpha}^{*^{\prime}}-\underline{y}_{j}^{\prime}\right) D_{j}^{-1} \underline{\beta}^{*}+\right.\right. \\
+ & \left.\left.\left.\underline{y_{j}^{\prime}} D_{j}^{-1} \underline{y_{j}}+\underline{\alpha}^{*^{\prime}} D_{j}^{-1} \underline{\alpha}^{*}-2 \underline{\alpha}^{*^{\prime}} D_{j}^{-1} \underline{y_{j}}\right)\right] / \Psi^{(0)}, Y^{*}\right] .
\end{aligned}
$$

Logo,

$$
\begin{aligned}
& E\left[L_{c}(\Psi) / \Psi^{(0)}, Y^{*}\right]=-\frac{(N M+1) P}{2} \ln (2 \pi)-\frac{1}{2} \sum_{j=1}^{P} \ln \left(\sigma_{x j}^{2} \sigma_{1 j}^{2} \ldots \sigma_{1 j}^{2} \ldots \sigma_{N j}^{2} \ldots \sigma_{N j}^{2}\right)- \\
& -\frac{1}{2} \sum_{j=1}^{P}\left[\frac{E\left[\left(x_{j} / \Psi^{(0)}, Y^{*}\right)-\mu_{j}\right]^{2}}{\sigma_{x j}^{2}}+E\left[x_{j}^{2} \underline{\beta}^{*^{\prime}} D_{j}^{-1} \underline{\beta}^{*} / \Psi^{(0)}, Y^{*}\right]+2 E\left[x_{j}\left(\underline{\alpha}^{*^{\prime}}-\underline{y}_{j}^{\prime}\right) D_{j}^{-1} \underline{\beta}^{*} / \Psi^{(0)}, Y^{*}\right]+\right. \\
& \left.+E\left[\underline{y}_{j}^{\prime} D_{j}^{-1} \underline{y_{j}} / \Psi^{(0)}, Y^{*}\right]+E\left[\underline{\alpha}^{*^{\prime}} D_{j}^{-1} \underline{\alpha}^{*} / \Psi^{(0)}, Y^{*}\right]-2 E\left[\underline{\alpha}^{*^{\prime}} D_{j}^{-1} \underline{y_{j}} / \Psi^{(0)}, Y^{*}\right]\right]
\end{aligned}
$$

Portanto, a equação acima pode ser reescrita na forma

$$
\begin{aligned}
E\left[L_{c}(\Psi) / \Psi^{(0)}, Y^{*}\right]=-\frac{(N M+1) P}{2} \ln (2 \pi)-\frac{1}{2} \sum_{j=1}^{P} \ln \left(\sigma_{x j}^{2} \sigma_{1 j}^{2} \ldots \sigma_{1 j}^{2} \ldots \sigma_{N j}^{2} \ldots \sigma_{N j}^{2}\right) \\
\quad-\frac{1}{2}\left[\sum _ { j = 1 } ^ { P } \left(\frac{\left(\hat{x}_{j}-\mu_{j}\right)^{2}}{\sigma_{x j}^{2}}+\hat{x}_{j}^{2} \underline{\beta}^{*^{\prime}} D_{j}^{-1} \underline{\beta}^{*}+2 \hat{x}_{j}\left(\underline{\alpha}^{*^{\prime}}-\underline{y}_{j}^{\prime}\right) D_{j}^{-1} \underline{\beta}^{*}+\right.\right. \\
\left.\left.+\underline{y}_{j}^{\prime} D_{j}^{-1} \underline{y_{j}}+\underline{\alpha}^{*^{\prime}} D_{j}^{-1} \underline{\alpha}^{*}-2 \underline{\alpha}^{*^{\prime}} D_{j}^{-1} \underline{y_{j}}\right)\right]
\end{aligned}
$$

onde $\hat{x}_{j}$ e $\hat{x}_{j}^{2}$ são dados pelas equações (3.8) e (3.9). Logo, ao maximizarmos a equação (3.20), obteremos as equações (3.16) e (3.17) dadas na seção 3.3.2.

\subsubsection{Convergência do Algoritmo EM}

Boyles (1983) afirma que as duas hipóteses do Teorema 2 enunciado em Dempster, Laird e Rubin (1977) não são suficientes para implicar que $\left\{L\left(\Psi^{(k)}\right)\right\}$ seja uma seqüência convergente, ou seja,

$$
\left\|\Psi^{(k+1)}-\Psi^{(k)}\right\| \rightarrow 0, \text { quando } k \rightarrow \infty .
$$

onde a norma dada acima é a norma euclidiana.

Para tanto, $\mathrm{Wu}$ (1983) estabelece um conjunto de suposições que garantem a convergência do algoritmo EM. Além disso, o artigo contém um estudo para se concluir sobre a natureza do limite da sequência acima (se o limite é um máximo global, máximo local ou ponto de sela de $L$ ).

A seguir, vamos estabelecer as condições que garantem a convergência do algoritmo EM e provar que estas condições valem para o caso de nossa função de verossimilhança.

Para garantirmos que $L\left(\Psi^{(k)}\right)$ converge monotonicamente para algum $L^{*}$, precisamos de duas condições (Wu, 1983, pg. 96, itens 3 e 8):

1. $L\left(\Psi^{(k+1)}\right) \geq L\left(\Psi^{(k)}\right)$; 
2. A sequência $\left\{L\left(\Psi^{(k)}\right)\right\}_{k \geq 0}$ é limitada superiormente para $\forall \Psi_{0} \in \Omega$.

Do item 1 temos que L não decresce após uma iteração do algoritmo EM (Dempster, Laird e Rubin, 1977, Teorema 1, pg.7).

Agora, para verificarmos o item 2, precisamos mostrar que a nossa função de verossimilhança (dados incompletos), dada na seção 3.2 (equação 3.2, pg. 27) é limitada superiormente. Ela é dada por

$$
f_{y}=\prod_{j=1}^{P} f_{y j}=(2 \pi)^{-\frac{N M P}{2}} \prod_{j=1}^{P}\left|V_{y j}\right|^{-\frac{1}{2}} \exp \left[-\frac{1}{2} \sum_{j=1}^{P}\left(y_{j}-\mu_{y j}\right)^{\prime} V_{y j}{ }^{-1}\left(y_{j}-\mu_{y j}\right)\right] .
$$

Notemos que a parte exponencial da função possui expoente negativo, logo ela é limitada superiormente. De fato, precisamos apenas mostrar que $\left|V_{y j}\right|^{-\frac{1}{2}}$ é também limitado superiormente. Temos que

$$
\left|V_{y j}\right|=\left[1+\sigma_{x j}^{2}\left(\frac{\beta_{1}^{2}}{\sigma_{1 j}^{2}}+\ldots+\frac{\beta_{1}^{2}}{\sigma_{1 j}^{2}}+\ldots+\frac{\beta_{N}^{2}}{\sigma_{N j}^{2}}+\ldots+\frac{\beta_{N}^{2}}{\sigma_{N j}^{2}}\right)\right] \sigma_{1 j}^{2} \ldots \sigma_{1 j}^{2} \ldots \sigma_{N j}^{2} \ldots \sigma_{N j}^{2}
$$

Então,

$$
\left|V_{y j}\right|^{-\frac{1}{2}}=\frac{1}{\sqrt{\left[1+\sigma_{x j}^{2}\left(\frac{\beta_{1}^{2}}{\sigma_{1 j}^{2}}+\ldots+\frac{\beta_{1}^{2}}{\sigma_{1 j}^{2}}+\ldots+\frac{\beta_{N}^{2}}{\sigma_{N j}^{2}}+\ldots+\frac{\beta_{N}^{2}}{\sigma_{N j}^{2}}\right)\right] \sigma_{1 j}^{2} \ldots \sigma_{1 j}^{2} \ldots \sigma_{N j}^{2} \ldots \sigma_{N j}^{2}}} .
$$

Como $\sigma_{x j}^{2}$ e os $\sigma_{i j}^{2}$ são fixados, precisamos apenas verificar os parâmetros $\beta_{i}, i=1, \ldots, N$. Para qualquer $\beta_{i}, i=1, \ldots, N$, temos que

- $\lim _{\beta_{i} \rightarrow 0}\left|V_{y j}\right|^{-\frac{1}{2}}=\frac{1}{\sqrt{\sigma_{1 j}^{2} \ldots \sigma_{1 j}^{2} \ldots \sigma_{N j}^{2} \ldots \sigma_{N j}^{2}}} ;$

- $\lim _{\beta_{i} \rightarrow-\infty}\left|V_{y j}\right|^{-\frac{1}{2}}=0$;

- $\lim _{\beta_{i} \rightarrow \infty}\left|V_{y j}\right|^{-\frac{1}{2}}=0, i=1, \ldots, N$.

ou seja, $\left|V_{y j}\right|^{-\frac{1}{2}}$ é limitado superiormente por $\frac{1}{\sqrt{\sigma_{1 j}^{2} \ldots \sigma_{1 j}^{2} \ldots \sigma_{N j}^{2} \ldots \sigma_{N j}^{2}}}, \forall j=1, \ldots, P$. Logo, $f_{y}$ é limitada superiormente.

Como L (Logaritmo natural) é uma função injetora, $L\left(f_{y}\right)$ também é limitada superiormente. Portanto, a sequência $\left\{L\left(\Psi^{(k)}\right)\right\}$ é limitada superiormente. De acordo com Wu (1983), pg. 97, item 2.1, $\left\{L\left(\Psi^{(k)}\right)\right\}$ converge monotonicamente para algum $L^{*}$.

O fato da sequência $\left\{L\left(\Psi^{(k)}\right)\right\}$ convergir monotonicamente para algum $L^{*}$ não garante que $L^{*}$ seja um ponto de máximo global de $L(\Psi)$ sobre $\Omega$. De fato, $L^{*}$ pode ser um ponto de máximo local ou ainda um ponto estacionário. No nosso caso, verificamos empiricamente que $L^{*}$ é um ponto de máximo global (ver Capítulo 4, Tabelas de 4.1 a 4.3), pois obtemos sempre o mesmo $L^{*}$ para diferentes valores iniciais para os parâmetros do modelo. 


\section{Capítulo 4}

\section{Aplicações}

\subsection{Introdução}

Neste capítulo, apresentamos aproximações para as estimativas de máxima verossimilhança para os parâmetros $\underline{\alpha}$ e $\beta$ obtidos via algoritmo EM (ver Apêndices C e D). Além disso, vamos propor uma estratégia para avaliarmos a compatibilidade e consistência dos laboratórios participantes do EP.

\subsection{Estimativas para os Parâmetros via Algoritmo EM}

Para avaliarmos a robustez do algoritmo EM, aplicamos o algoritmo EM para diferentes valores iniciais dos parâmetros.

\begin{tabular}{|c|c|c|c|c|}
\hline Parâmetros & Valores Iniciais & Estimativas & $\mathrm{N}^{\mathbf{0}}$ de Iterações & $L^{*}$ \\
\hline$\alpha_{1}$ & 0 & 0.3002 & \multirow{22}{*}{5} & \multirow{22}{*}{-383.1246} \\
\hline$\alpha_{2}$ & 0 & 0.0542 & & \\
\hline$\alpha_{3}$ & 0 & -0.2264 & & \\
\hline$\alpha_{4}$ & 0 & 0.2769 & & \\
\hline$\alpha_{5}$ & 0 & -0.0668 & & \\
\hline$\alpha_{6}$ & 0 & -1.6080 & & \\
\hline$\alpha_{7}$ & 0 & -1.6222 & & \\
\hline$\alpha_{8}$ & 0 & 0.0789 & & \\
\hline$\alpha_{9}$ & 0 & -0.1328 & & \\
\hline$\alpha_{10}$ & 0 & -0.7009 & & \\
\hline$\alpha_{11}$ & 0 & 0.0289 & & \\
\hline$\beta_{1}$ & 0 & -0.0109 & & \\
\hline$\beta_{2}$ & 0 & -0.0063 & & \\
\hline$\beta_{3}$ & 0 & 0.0028 & & \\
\hline$\beta_{4}$ & 0 & -0.0085 & & \\
\hline$\beta_{5}$ & 0 & -0.0078 & & \\
\hline$\beta_{6}$ & 0 & -0.0003 & & \\
\hline$\beta_{7}$ & 0 & -0.0003 & & \\
\hline$\beta_{8}$ & 0 & -0.0043 & & \\
\hline$\beta_{9}$ & 0 & 0.0037 & & \\
\hline$\beta_{10}$ & 0 & -0.0014 & & \\
\hline$\beta_{11}$ & 0 & -0.0004 & & \\
\hline
\end{tabular}

Tabela 4.1: Teste 1 - Valores das Estimativas para os Parâmetros via Algoritmo EM

Seqüência $\left\{L\left(\Psi^{(k)}\right)\right\}:(-415.2326,-383.1215,-383.1246,-383.1246,-383.1246)$. 


\begin{tabular}{|c|c|c|c|c|}
\hline Parâmetros & Valores Iniciais & Estimativas & $\mathrm{N}^{\mathrm{O}}$ de Iterações & $L^{*}$ \\
\hline$\alpha_{1}$ & 0.56 & 0.3002 & \multirow{22}{*}{7} & \multirow{22}{*}{-383.1246} \\
\hline$\alpha_{2}$ & -0.99 & 0.0542 & & \\
\hline$\alpha_{3}$ & 1 & -0.2264 & & \\
\hline$\alpha_{4}$ & 0.13 & 0.2769 & & \\
\hline$\alpha_{5}$ & 1.6 & -0.0668 & & \\
\hline$\alpha_{6}$ & -1 & -1.6080 & & \\
\hline$\alpha_{7}$ & 0.549 & -1.6222 & & \\
\hline$\alpha_{8}$ & -0.954 & 0.0789 & & \\
\hline$\alpha_{9}$ & 1.12 & -0.1328 & & \\
\hline$\alpha_{10}$ & -1.5 & -0.7009 & & \\
\hline$\alpha_{11}$ & 0.8 & 0.0289 & & \\
\hline$\beta_{1}$ & 0 & -0.0109 & & \\
\hline$\beta_{2}$ & 1 & -0.0063 & & \\
\hline$\beta_{3}$ & 0.98 & 0.0028 & & \\
\hline$\beta_{4}$ & 0.537 & -0.0085 & & \\
\hline$\beta_{5}$ & -0.89 & -0.0078 & & \\
\hline$\beta_{6}$ & 1.3 & -0.0003 & & \\
\hline$\beta_{7}$ & -0.67 & -0.0003 & & \\
\hline$\beta_{8}$ & 0.088 & -0.0043 & & \\
\hline$\beta_{9}$ & -1.2 & 0.0037 & & \\
\hline$\beta_{10}$ & 1.804 & -0.0014 & & \\
\hline$\beta_{11}$ & -0.556 & -0.0004 & & \\
\hline
\end{tabular}

Tabela 4.2: Teste 2 - Valores das Estimativas para os Parâmetros via Algoritmo EM

Seqüência $\left\{L\left(\Psi^{(k)}\right)\right\}$ : $(-1542729.1648,-304038.2122,-4467.8474,-385.7360$, $-383.1280,-383.1246,-383.1246)$. 


\begin{tabular}{|c|c|c|c|c|}
\hline Parâmetros & Valores Iniciais & Estimativas & $\mathbf{N}^{\mathbf{0}}$ de Iterações & $L^{*}$ \\
\hline$\alpha_{1}$ & 23 & 0.3002 & \multirow{22}{*}{9} & \multirow{22}{*}{-383.1246} \\
\hline$\alpha_{2}$ & 52 & 0.0542 & & \\
\hline$\alpha_{3}$ & 160 & -0.2264 & & \\
\hline$\alpha_{4}$ & 100 & 0.2769 & & \\
\hline$\alpha_{5}$ & 46 & -0.0668 & & \\
\hline$\alpha_{6}$ & -37 & -1.6080 & & \\
\hline$\alpha_{7}$ & -9 & -1.6222 & & \\
\hline$\alpha_{8}$ & -648 & 0.0789 & & \\
\hline$\alpha_{9}$ & 25 & -0.1328 & & \\
\hline$\alpha_{10}$ & 891 & -0.7009 & & \\
\hline$\alpha_{11}$ & -184 & 0.0289 & & \\
\hline$\beta_{1}$ & 101 & -0.0109 & & \\
\hline$\beta_{2}$ & 194 & -0.0063 & & \\
\hline$\beta_{3}$ & -90 & 0.0028 & & \\
\hline$\beta_{4}$ & -307 & -0.0085 & & \\
\hline$\beta_{5}$ & 24 & -0.0078 & & \\
\hline$\beta_{6}$ & 91 & -0.0003 & & \\
\hline$\beta_{7}$ & 55 & -0.0003 & & \\
\hline$\beta_{8}$ & 62 & -0.0043 & & \\
\hline$\beta_{9}$ & 218 & 0.0037 & & \\
\hline$\beta_{10}$ & -75 & -0.0014 & & \\
\hline$\beta_{11}$ & 456 & -0.0004 & & \\
\hline
\end{tabular}

Tabela 4.3: Teste 3 - Valores das Estimativas para os Parâmetros via Algoritmo EM

Seqüência $\left\{L\left(\Psi^{(k)}\right)\right\}$ : $(-21217327795.5909,-4115593.3685,-1594890.2128$, $-110966.6056,-829.6552,-383.3749,-383.1250,-383.1246,-383.1246)$.

Pelos resultados apresentados nas Tabelas 4.1, 4.2 e 4.3, podemos concluir que o algoritmo EM é consistente e robusto em relação aos valores iniciais atribuídos aos parâmetros $\underline{\alpha}$ e $\underline{\beta}$, mudando somente o número de iterações necessárias para se obter uma aproximação para as estimativas de máxima verossimilhança para os parâmetros.

\subsection{Análise da Competência dos Laboratórios}

Como o nosso modelo, que descreve os dados provenientes de um EP não podem ser organizados em vetores aleatórios independentes e identicamente distribuídos, não conseguimos aplicar os resultados clássicos da teoria assintótica (referente aos testes de hipóteses)(ver Lehmann, 1983, Teorema 4.1, pg. 429 e Sen e Singer, 1993, Teorema 5.2.1, pg. 205).

Nossa estratégia de análise neste caso, foi obter "intervalos de confiança" a 95\% para a tendência $Y$ em cada laboratório e em cada patamar de temperatura, via simulação. Para tanto, realizamos os seguintes passos:

1. Fornecemos as aproximações para as estimativas de máxima verossimilhança de $\alpha_{i} \mathrm{e}$ $\beta_{i}$, obtidos via algoritmo EM;

2. Geramos 1000 valores de erros $\epsilon_{i j k} \sim N\left(0, \sigma_{i j}^{2}\right)$;

3. Geramos 1000 valores para a variável explicativa $X_{j} \sim N\left(\mu_{j}, \sigma_{x j}^{2}\right)$;

4. Encontramos $Y_{i j k}=\alpha_{i}+\beta_{i} X_{j}+\epsilon_{i j k}$

5. Ordenamos $Y_{i j k}$ e tomamos as posições 25 e 975 a fim de obtermos um intervalo com $95 \%$ de confiança. 
Obs.: A rotina para os cálculos dos intevalos de confiança encontra-se no Apêndice E apenas para o laboratório 1. As outras rotinas são similares, apenas mudando os valores para as aproximações das estimativas de máxima verossimilhança dos parâmetros e as variâncias.

Os intervalos e respectivos gráficos são dados a seguir.

\section{Laboratório 1}

\begin{tabular}{|c|c|}
\hline Patamares & \multicolumn{1}{|c|}{ Intervalos } \\
\hline \hline $25^{\circ} \mathrm{C}$ & {$[-0.78561 ; 0.75506]$} \\
\hline $50^{\circ} \mathrm{C}$ & {$[-1.1627 ; 0.70719]$} \\
\hline $175^{\circ} \mathrm{C}$ & {$[-2.5022 ;-0.71651]$} \\
\hline $300^{\circ} \mathrm{C}$ & {$[-4.1655 ;-1.7229]$} \\
\hline $425^{\circ} \mathrm{C}$ & {$[-5.3838 ;-3.2649]$} \\
\hline
\end{tabular}

Tabela 4.4: Intervalos de $95 \%$ de confiança para a tendência $\left({ }^{\circ} C\right)$ - Laboratório 1

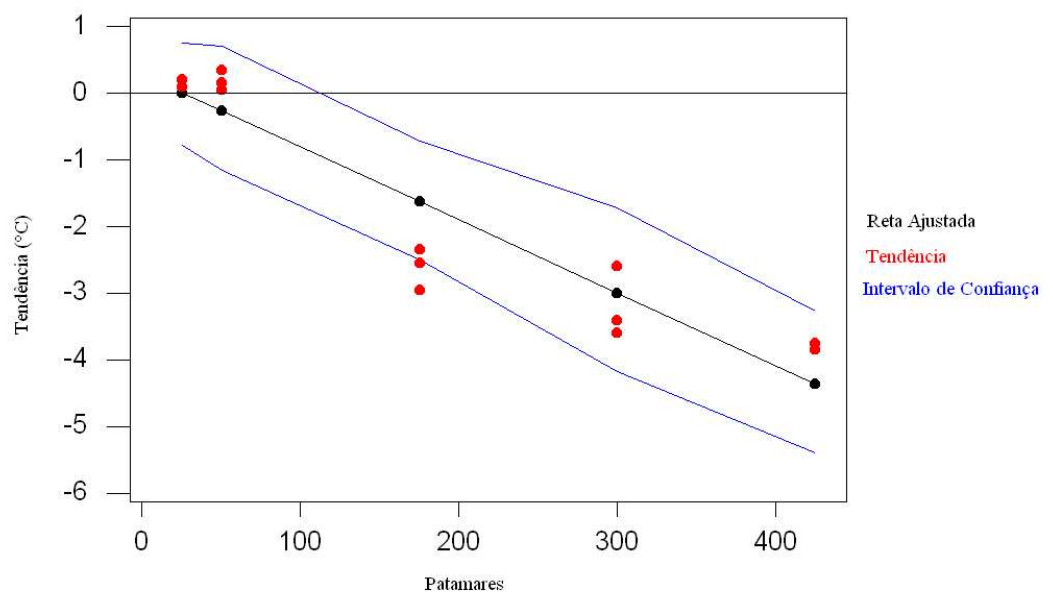

Figura 4.1: Tendência, Reta Ajustada e Intervalo de Confiança para o laboratório 1

\section{Laboratório 2}

\begin{tabular}{|c|l|}
\hline Patamares & \multicolumn{1}{|c|}{ Intervalos } \\
\hline \hline $25^{\circ} \mathrm{C}$ & {$[-1.467 ; 1.342]$} \\
\hline $50^{\circ} \mathrm{C}$ & {$[-1.6717 ; 1.2566]$} \\
\hline $175^{\circ} \mathrm{C}$ & {$[-2.5287 ; 0.39721]$} \\
\hline $300^{\circ} \mathrm{C}$ & {$[-3.194 ;-0.34623]$} \\
\hline $425^{\circ} \mathrm{C}$ & {$[-4.9261 ;-0.40429]$} \\
\hline
\end{tabular}

Tabela 4.5: Intervalos de $95 \%$ de confiança para a tendência $\left({ }^{\circ} C\right)$ - Laboratório 2 


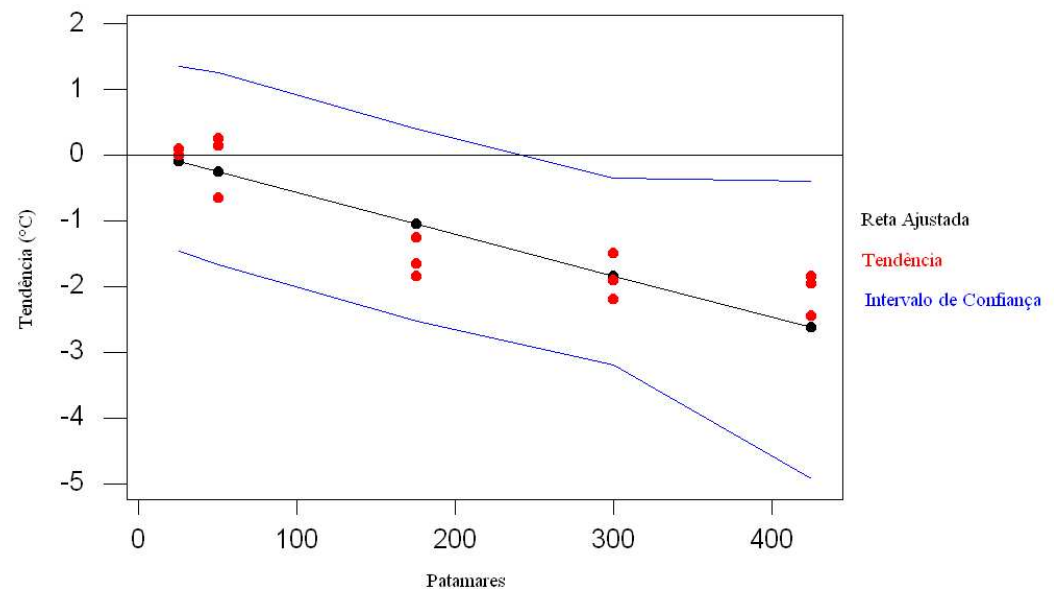

Figura 4.2: Tendência, Reta Ajustada e Intervalo de Confiança para o laboratório 2

\section{Laboratório 3}

\begin{tabular}{|c|l|}
\hline Patamares & \multicolumn{1}{|c|}{ Intervalos } \\
\hline \hline $25^{\circ} \mathrm{C}$ & {$[-0.58101 ; 0.26246]$} \\
\hline $50^{\circ} \mathrm{C}$ & {$[-0.46708 ; 0.28588]$} \\
\hline $175^{\circ} \mathrm{C}$ & {$[-0.6392 ; 1.1219]$} \\
\hline $300^{\circ} \mathrm{C}$ & {$[-0.14489 ; 1.3619]$} \\
\hline $425^{\circ} \mathrm{C}$ & {$[0.35351 ; 1.5589]$} \\
\hline
\end{tabular}

Tabela 4.6: Intervalos de $95 \%$ de confiança para a tendência $\left({ }^{\circ} C\right)$ - Laboratório 3

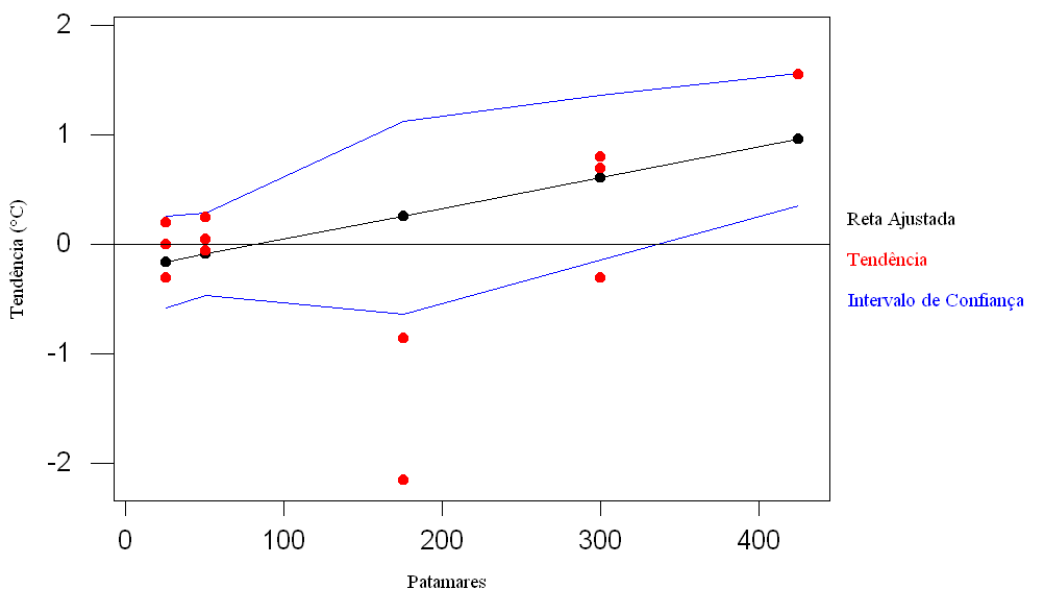

Figura 4.3: Tendência, Reta Ajustada e Intervalo de Confiança para o laboratório 3 


\section{Laboratório 4}

\begin{tabular}{|c|l|}
\hline Patamares & \multicolumn{1}{|c|}{ Intervalos } \\
\hline \hline $25^{\circ} \mathrm{C}$ & {$[0.1164 ; 0.57712]$} \\
\hline $50^{\circ} \mathrm{C}$ & {$[-0.0053129 ; 0.48279]$} \\
\hline $175^{\circ} \mathrm{C}$ & {$[-0.62529 ;-0.097991]$} \\
\hline $300^{\circ} \mathrm{C}$ & {$[-1.5557 ;-0.3003]$} \\
\hline $425^{\circ} \mathrm{C}$ & {$[-2.0897 ;-0.90077]$} \\
\hline
\end{tabular}

Tabela 4.7: Intervalos de $95 \%$ de confiança para a tendência $\left({ }^{\circ} C\right)$ - Laboratório 4

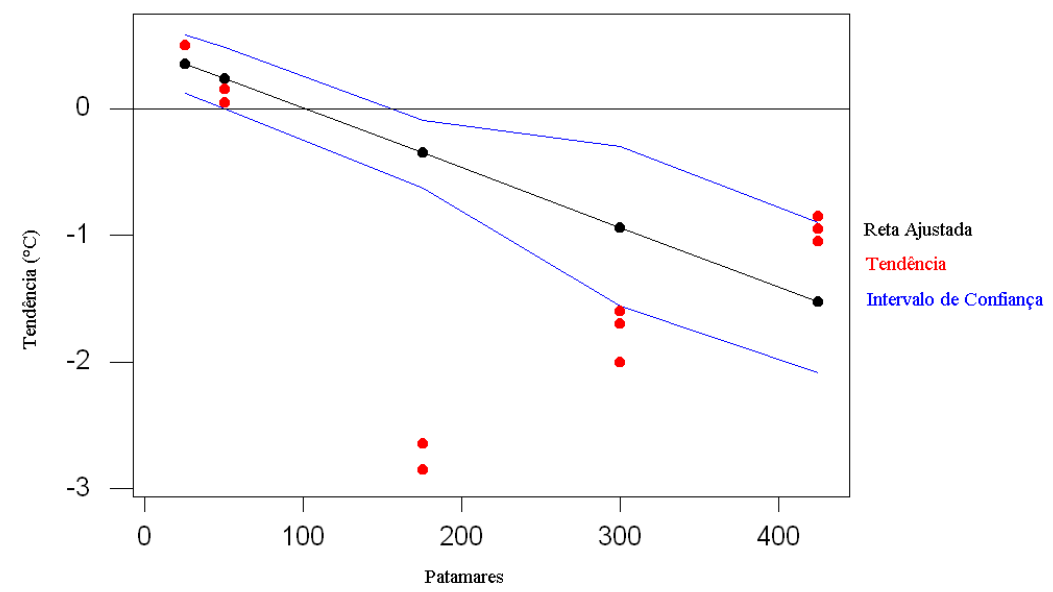

Figura 4.4: Tendência, Reta Ajustada e Intervalo de Confiança para o laboratório 4

\section{Laboratório 5}

\begin{tabular}{|c|c|}
\hline Patamares & Intervalos \\
\hline \hline $25^{\circ} \mathrm{C}$ & {$[-0.4746 ;-0.062072]$} \\
\hline $50^{\circ} \mathrm{C}$ & {$[-0.68889 ;-0.20616]$} \\
\hline $175^{\circ} \mathrm{C}$ & {$[-1.7089 ;-1.1636]$} \\
\hline $300^{\circ} \mathrm{C}$ & {$[-2.9745 ;-1.8787]$} \\
\hline $425^{\circ} \mathrm{C}$ & {$[-4.0445 ;-2.7013]$} \\
\hline
\end{tabular}

Tabela 4.8: Intervalos de $95 \%$ de confiança para a tendência $\left({ }^{\circ} C\right)$ - Laboratório 5 


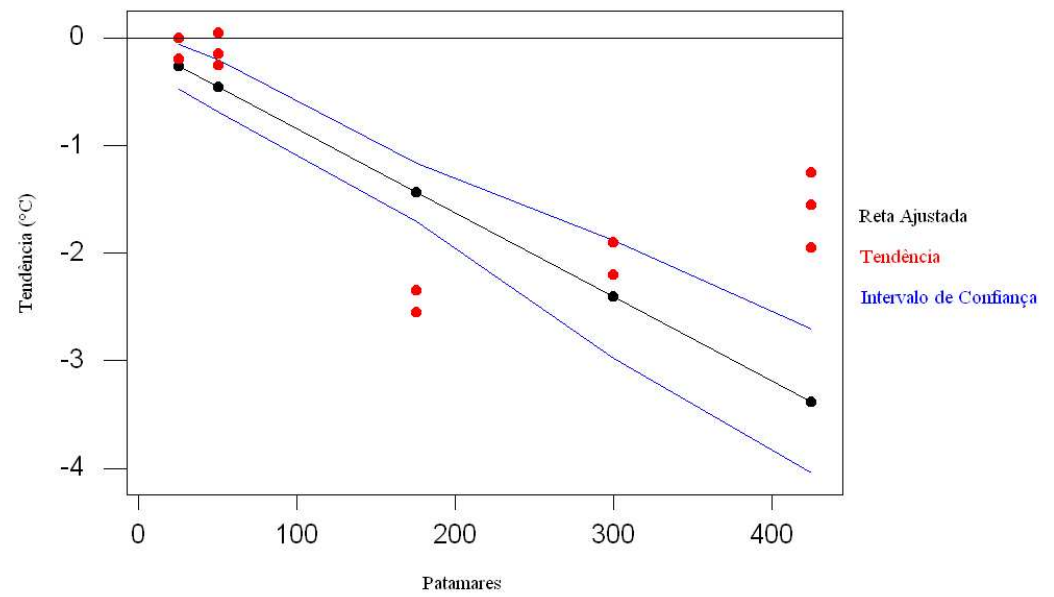

Figura 4.5: Tendência, Reta Ajustada e Intervalo de Confiança para o laboratório 5

\section{Laboratório 6}

\begin{tabular}{|c|c|}
\hline Patamares & Intervalos \\
\hline \hline $25^{\circ} \mathrm{C}$ & {$[-1.8302 ;-1.3946]$} \\
\hline $50^{\circ} \mathrm{C}$ & {$[-1.838 ;-1.4107]$} \\
\hline $175^{\circ} \mathrm{C}$ & {$[-1.9287 ;-1.3739]$} \\
\hline $300^{\circ} \mathrm{C}$ & {$[-2.1942 ;-1.2172]$} \\
\hline $425^{\circ} \mathrm{C}$ & {$[-2.1067 ;-1.3582]$} \\
\hline
\end{tabular}

Tabela 4.9: Intervalos de $95 \%$ de confiança para a tendência $\left({ }^{\circ} C\right)$ - Laboratório 6

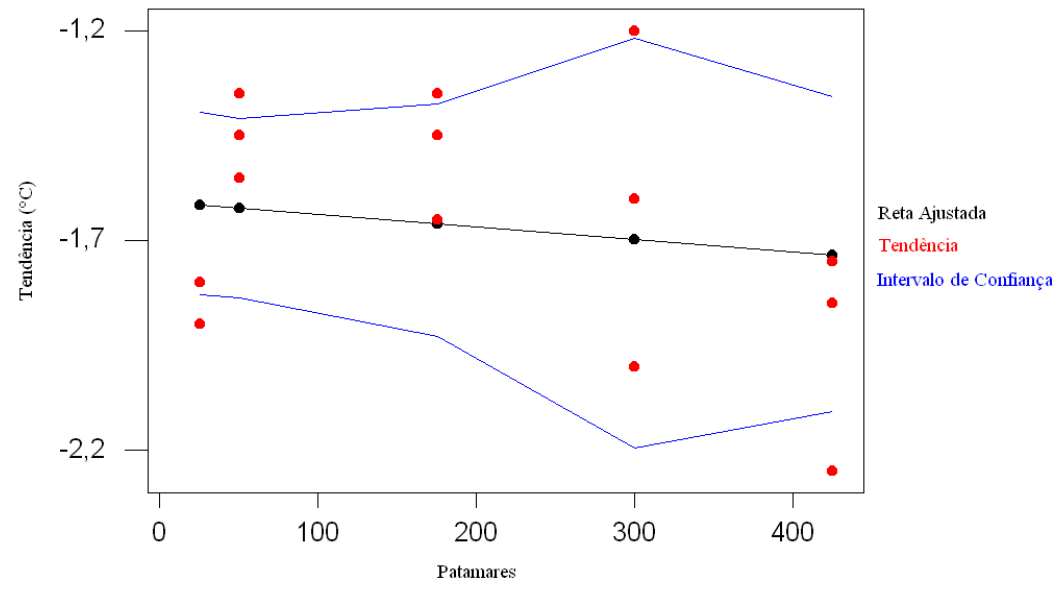

Figura 4.6: Tendência, Reta Ajustada e Intervalo de Confiança para o laboratório 6 


\section{Laboratório 7}

\begin{tabular}{|c|c|}
\hline Patamares & \multicolumn{1}{|c|}{ Intervalos } \\
\hline \hline $25^{\circ} \mathrm{C}$ & {$[-1.8536 ;-1.4161]$} \\
\hline $50^{\circ} \mathrm{C}$ & {$[-1.8758 ;-1.399]$} \\
\hline $175^{\circ} \mathrm{C}$ & {$[-1.9663 ;-1.42]$} \\
\hline $300^{\circ} \mathrm{C}$ & {$[-2.1909 ;-1.2058]$} \\
\hline $425^{\circ} \mathrm{C}$ & {$[-2.111 ;-1.3734]$} \\
\hline
\end{tabular}

Tabela 4.10: Intervalos de $95 \%$ de confiança para a tendência $\left({ }^{\circ} C\right)$ - Laboratório 7

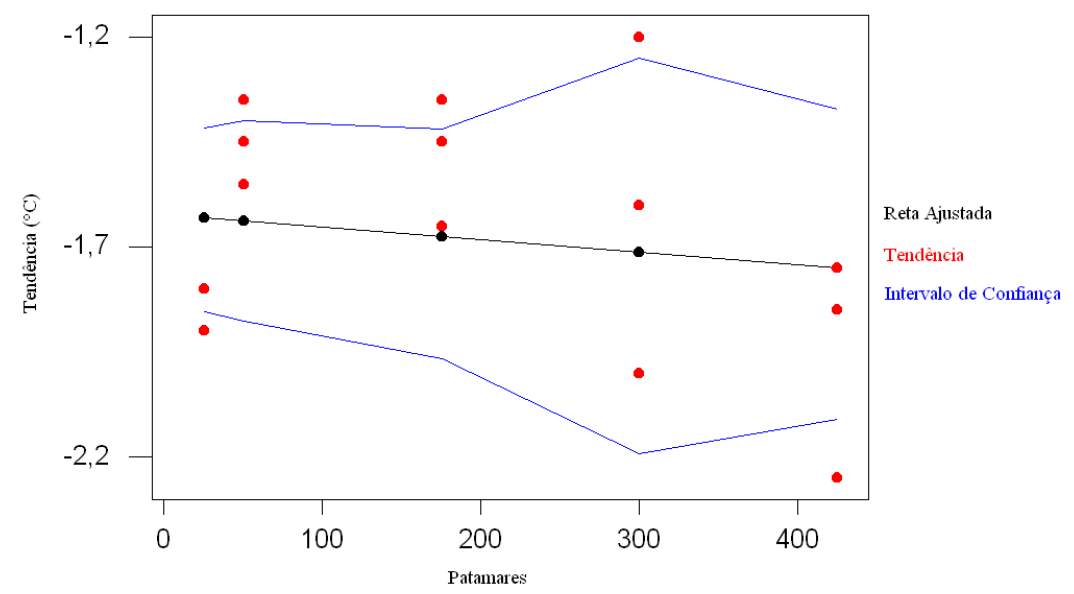

Figura 4.7: Tendência, Reta Ajustada e Intervalo de Confiança para o laboratório 7

\section{Laboratório 8}

\begin{tabular}{|c|c|}
\hline Patamares & Intervalos \\
\hline \hline $25^{\circ} \mathrm{C}$ & {$[-0.092915 ; 0.034028]$} \\
\hline $50^{\circ} \mathrm{C}$ & {$[-0.20481 ;-0.071692]$} \\
\hline $175^{\circ} \mathrm{C}$ & {$[-0.99337 ;-0.34351]$} \\
\hline $300^{\circ} \mathrm{C}$ & {$[-1.8062 ;-0.64336]$} \\
\hline $425^{\circ} \mathrm{C}$ & {$[-2.3601 ;-1.1492]$} \\
\hline
\end{tabular}

Tabela 4.11: Intervalos de $95 \%$ de confiança para a tendência $\left({ }^{\circ} C\right)$ - Laboratório 8 


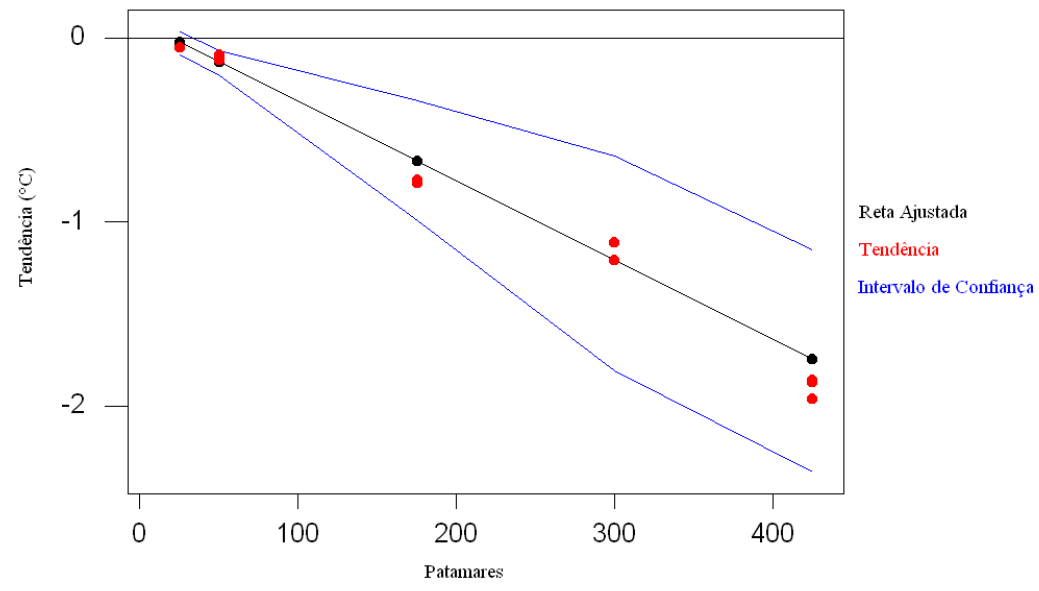

Figura 4.8: Tendência, Reta Ajustada e Intervalo de Confiança para o laboratório 8

Laboratório 9

\begin{tabular}{|c|l|}
\hline Patamares & \multicolumn{1}{|c|}{ Intervalos } \\
\hline \hline $25^{\circ} \mathrm{C}$ & {$[-0.28748 ; 0.1792]$} \\
\hline $50^{\circ} \mathrm{C}$ & {$[-0.12145 ; 0.21987]$} \\
\hline $175^{\circ} \mathrm{C}$ & {$[0.37324 ; 0.66336]$} \\
\hline $300^{\circ} \mathrm{C}$ & {$[0.83736 ; 1.1253]$} \\
\hline $425^{\circ} \mathrm{C}$ & {$[1.2073 ; 1.6832]$} \\
\hline
\end{tabular}

Tabela 4.12: Intervalos de $95 \%$ de confiança para a tendência $\left({ }^{\circ} C\right)$ - Laboratório 9

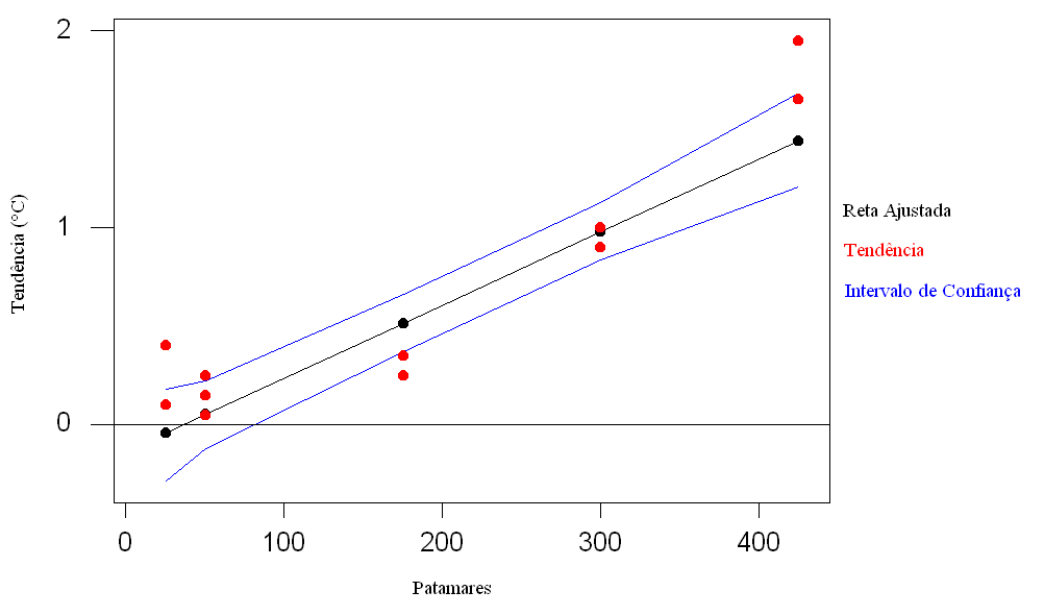

Figura 4.9: Tendência, Reta Ajustada e Intervalo de Confiança para o laboratório 9 


\section{Laboratório 10}

\begin{tabular}{|c|c|}
\hline Patamares & \multicolumn{1}{|c|}{ Intervalos } \\
\hline \hline $25^{\circ} \mathrm{C}$ & {$[-1.0184 ;-0.47797]$} \\
\hline $50^{\circ} \mathrm{C}$ & {$[-1.9674 ; 0.2711]$} \\
\hline $175^{\circ} \mathrm{C}$ & {$[-1.136 ;-0.76228]$} \\
\hline $300^{\circ} \mathrm{C}$ & {$[-2.9547 ; 0.64302]$} \\
\hline $425^{\circ} \mathrm{C}$ & {$[-2.7577 ; 0.17854]$} \\
\hline
\end{tabular}

Tabela 4.13: Intervalos de $95 \%$ de confiança para a tendência $\left({ }^{\circ} C\right)$ - Laboratório 10

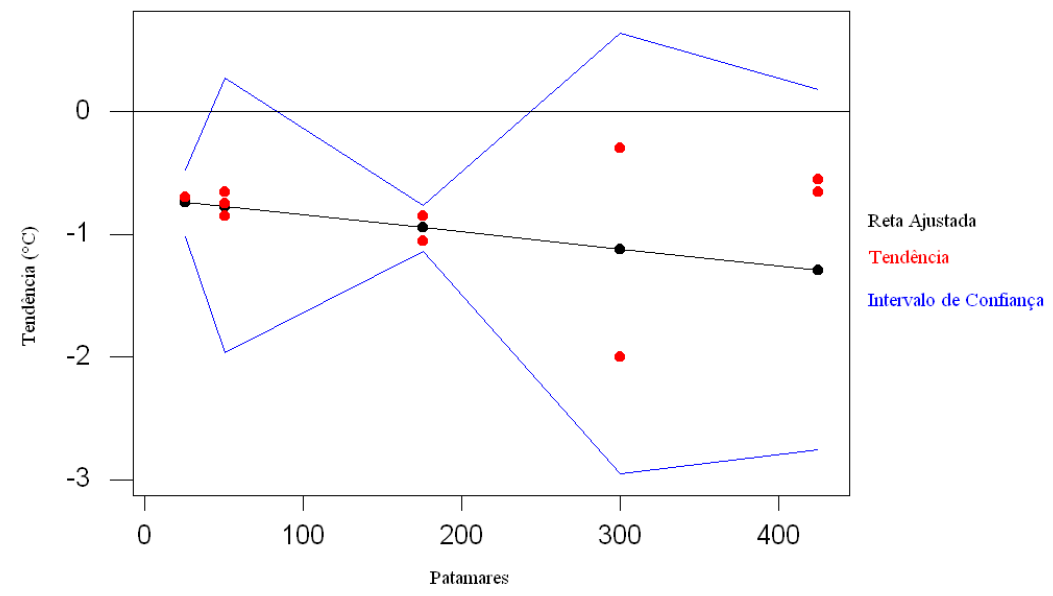

Figura 4.10: Tendência, Reta Ajustada e Intervalo de Confiança para o laboratório 10

\section{Laboratório 11}

\begin{tabular}{|c|c|}
\hline Patamares & Intervalos \\
\hline \hline $25^{\circ} \mathrm{C}$ & {$[-0.13238 ; 0.16102]$} \\
\hline $50^{\circ} \mathrm{C}$ & {$[-0.15047 ; 0.16483]$} \\
\hline $175^{\circ} \mathrm{C}$ & {$[-0.18858 ; 0.099791]$} \\
\hline $300^{\circ} \mathrm{C}$ & {$[-0.26292 ; 0.10507]$} \\
\hline $425^{\circ} \mathrm{C}$ & {$[-0.5288 ; 0.2344]$} \\
\hline
\end{tabular}

Tabela 4.14: Intervalos de $95 \%$ de confiança para a tendência $\left({ }^{\circ} C\right)$ - Laboratório 11 


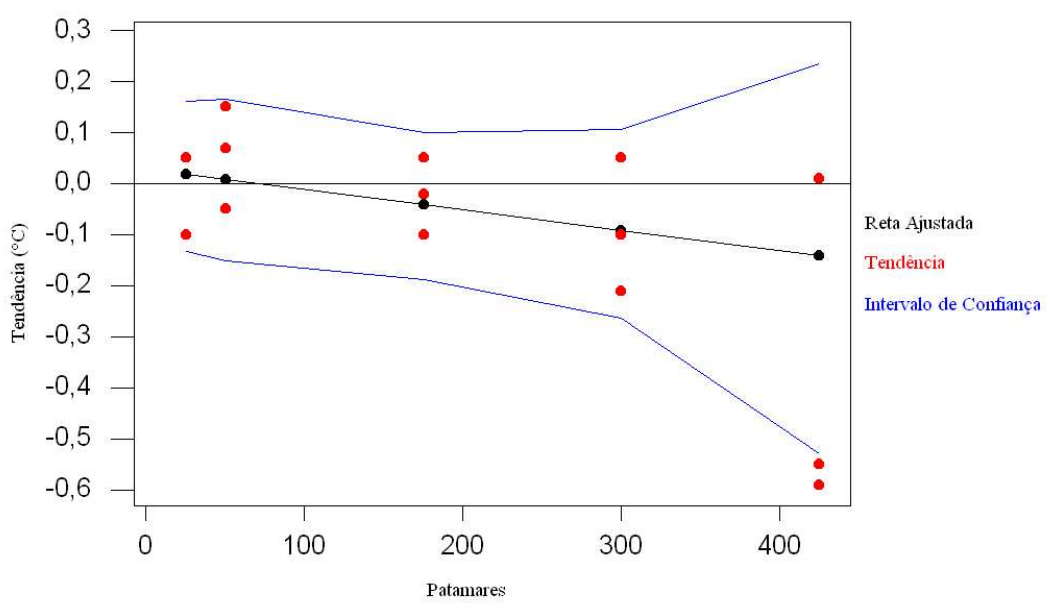

Figura 4.11: Tendência, Reta Ajustada e Intervalo de Confiança para o laboratório 11

Como podemos observar pelos gráficos acima, apenas o laboratório 11 pode ser considerado competente pois é o único cujo intervalo de confiança contém o zero em todos os patamares de temperatura analisados, ou seja, temos indícios que suas medições podem ser considerados " similares" ao valor de referência (VR) a menos de um erro. 


\section{Capítulo 5}

\section{Considerações Finais}

\subsection{Conclusões}

O objetivo do programa de ensaios de proficiência é qualificar ou não os laboratórios participantes. Analisamos os dados obtidos pelo Grupo de Motores da área de temperatura, cujo mensurando é um termopar com indicador de temperatura. Nesse sentido, nosso objetivo foi analisar a competência dos laboratórios participantes.

Para tal análise, modelamos os dados utilizando a distribuição normal. Consideramos, ainda, um modelo com erros de medição e variável explicativa aleatória. Diferente de outros modelos, estamos interessados em comparar a tendência dos laboratórios participantes em relação a um valor de referência, que é determinado por dois laboratórios que pertencem à Rede Brasileira de Calibração (RBC).

Neste modelo, estávamos interessados em encontrar os estimadores de máxima verossimilhança dos parâmetros $\underline{\alpha}$ e $\beta$ e propor um teste para testar a competência dos laboratórios.

Na prática, não conseguimos encontrar explicitamente os estimadores de $\underline{\alpha}$ e $\beta$. Como método alternativo, utilizamos o algoritmo EM para encontrar aproximações para as estimativas de máxima verossimilhança dos parâmetros e estudamos a sua convergência.

Pelo fato de não ser possível dispor nossos vetores de observações de modo a serem independentes e identicamente distribuídos, não conseguimos aplicar os resultados clássicos da teoria assintótica (ver Lehmann, 1983, Teorema 4.1, pg. 429 e Sen e Singer, 1993, Teorema 5.2.1, pg. 205). Desse modo, a estratégia foi obter intervalos de confiança a $95 \%$ para as tendências $Y$ via simulação para cada laboratório em cada patamar de temperatura.

\subsection{Propostas de Trabalhos Futuros}

- Desenvolver um teste para testar a consistência do grupo de laboratórios e a competência dos laboratórios individualmente;

- Estudar condições sobre o algoritmo EM para se obter convergência cujo limite seja um máximo global;

- Avaliar a consistência dos estimadores de máxima verossimilhança. 


\section{Apêndice A}

\section{Dados dos Laboratórios}

\begin{tabular}{|c|c|c|c|c|c|c|c|c|c|c|c|c|c|c|c|c|}
\hline \multicolumn{17}{|c|}{ Calibração } \\
\hline $\begin{array}{l}\text { F.M. } \\
\left({ }^{\circ} \mathrm{C}\right)\end{array}$ & Art. & $\begin{array}{l}1 \\
\text { E.M.L. }\end{array}$ & D & Art. & $\begin{array}{l}1 \\
\text { E.M.L. }\end{array}$ & D & Art. & $\begin{array}{l}2 \\
\text { E.M.L. }\end{array}$ & D & $\begin{array}{c}\text { med / } \\
\text { tend }\end{array}$ & $\begin{array}{l}\text { Desvio } \\
\text { Padrão }\end{array}$ & DPM & $\begin{array}{c}\mathrm{UE} \\
\mathrm{T}\end{array}$ & $\begin{array}{l}\mathrm{K} \\
\mathrm{T}\end{array}$ & $\begin{array}{l}\text { UE } \\
\text { Ind. }\end{array}$ & $\begin{array}{c}\mathrm{K} \\
\text { Ind. }\end{array}$ \\
\hline 25 & 26,5 & 24,8 & 1,7 & 26,6 & 24,8 & 1,8 & 26,6 & 24,8 & 1,8 & 1,8 & 0,058 & 0,033 & 1,4 & 2 & 0,2 & 2 \\
\hline 50 & 51,4 & 49,6 & 1,8 & 51,5 & 49,6 & 1,9 & 50,6 & 49,6 & 1 & 1,6 & 0,493 & 0,285 & 1,4 & 2 & 0,2 & 2 \\
\hline 175 & 173,3 & 173,4 & $-0,1$ & 175,6 & 175,3 & 0,3 & 173,2 & 173,5 & $-0,3$ & 0,0 & 0,306 & 0,176 & 1,4 & 2 & 0,2 & 2 \\
\hline 300 & 299,1 & 300,1 & $-1,0$ & 302,8 & 303,1 & $-0,3$ & 300,9 & 301,6 & $-0,7$ & $-0,7$ & 0,351 & 0,203 & 1,4 & 2 & 0,2 & 2 \\
\hline 425 & 425,4 & 426,7 & $-1,3$ & 425,6 & 426,3 & $-0,7$ & 424,7 & 425,5 & $-0,8$ & $-0,9$ & 0,321 & 0,186 & 2,4 & 2 & 0,2 & 2 \\
\hline
\end{tabular}

Tabela A.1: Dados do laboratório 2

\begin{tabular}{|c|c|c|c|c|c|c|c|c|c|c|c|c|c|c|c|c|}
\hline \multicolumn{17}{|c|}{ Calibração } \\
\hline $\begin{array}{l}\text { F.M. } \\
\left({ }^{\circ} \mathrm{C}\right)\end{array}$ & Art. & $\begin{array}{l}1 \\
\text { E.M.L. }\end{array}$ & D & Art. & $\begin{array}{l}1 \\
\text { E.M.L. }\end{array}$ & D & Art. & $\begin{array}{l}2 \\
\text { E.M.L. }\end{array}$ & D & $\begin{array}{c}\text { med / } \\
\text { tend }\end{array}$ & $\begin{array}{l}\text { Desvio } \\
\text { Padrão } \\
\end{array}$ & DPM & $\begin{array}{l}\mathrm{UE} \\
\mathrm{T}\end{array}$ & $\begin{array}{l}\mathrm{K} \\
\mathrm{T} \\
\end{array}$ & $\begin{array}{l}\text { UE } \\
\text { Ind. }\end{array}$ & $\begin{array}{c}\mathrm{K} \\
\text { Ind. }\end{array}$ \\
\hline 25 & 27,2 & 25,3 & 1,9 & 25,1 & 23,7 & 1,4 & 25,6 & 23,9 & 1,7 & 1,7 & 0,252 & 0,145 & 0,3 & 2 & 0,1 & 2 \\
\hline 50 & 52,2 & 50,3 & 1,9 & 50,5 & 48,9 & 1,6 & 50,1 & 48,4 & 1,7 & 1,7 & 0,153 & 0,088 & 0,3 & 2 & 0,1 & 2 \\
\hline 175 & 169,3 & 169,9 & $-0,6$ & 174,4 & 173,7 & 0,7 & 175,4 & 176,0 & $-0,6$ & $-0,2$ & 0,751 & 0,433 & 0,3 & 2 & 0,1 & 2 \\
\hline 300 & 300,9 & 299,0 & 1,9 & 300,1 & 299,2 & 0,9 & 298,5 & 296,5 & 2 & 1,6 & 0,608 & 0,351 & 0,3 & 2 & 0,1 & 2 \\
\hline 425 & 426,1 & 423,4 & 2,7 & 425,5 & 422,8 & 2,7 & 425,0 & 422,3 & 2,7 & 2,7 & 0,0 & 0,0 & 0,6 & 2 & 0,1 & 2 \\
\hline
\end{tabular}

Tabela A.2: Dados do laboratório 3

\begin{tabular}{|c|c|c|c|c|c|c|c|c|c|c|c|c|c|c|c|c|}
\hline \multicolumn{17}{|c|}{ Calibração } \\
\hline $\begin{array}{l}\text { F.M. } \\
\left({ }^{\circ} \mathrm{C}\right)\end{array}$ & \multicolumn{2}{|c|}{ A 1} & D & \multicolumn{2}{|c|}{ R 1} & D & \multicolumn{2}{|c|}{ A 2} & D & $\begin{array}{c}\text { med / } \\
\text { tend }\end{array}$ & $\begin{array}{l}\text { Desvio } \\
\text { Padrão }\end{array}$ & DPM & $\begin{array}{c}\mathrm{UE} \\
\mathrm{T}\end{array}$ & $\begin{array}{l}\mathrm{K} \\
\mathrm{T} \\
\end{array}$ & $\begin{array}{l}\text { UE } \\
\text { Ind. }\end{array}$ & $\begin{array}{r}\mathrm{K} \\
\text { Ind } \\
\end{array}$ \\
\hline 25 & 22,2 & 20,0 & 2,2 & 23,6 & 21,4 & 2,2 & 23,7 & 21,5 & 2,2 & 2,2 & $3 \mathrm{E}-8$ & $2 \mathrm{E}-8$ & 0,2 & 2 & 0,1 & 2 \\
\hline 50 & 50,6 & 48,8 & 1,8 & 51,9 & 50,1 & 1,8 & 51,6 & 49,9 & 1,7 & 1,8 & 0,058 & 0,033 & 0,2 & 2 & 0,1 & 2 \\
\hline 175 & 175,0 & 176,1 & $-1,1$ & 174,4 & 175,5 & $-1,1$ & 175,1 & 176,4 & $-1,3$ & $-1,2$ & 0,115 & 0,067 & 0,2 & 2 & 0,1 & 2 \\
\hline 300 & 300,0 & 300,4 & $-0,4$ & 300,0 & 300,5 & $-0,5$ & 299,3 & 300,1 & $-0,8$ & $-0,6$ & 0,208 & 0,12 & 0,6 & 2 & 0,1 & 2 \\
\hline 425 & 425,0 & 424,8 & 0,2 & 425,0 & 424,7 & 0,3 & 425,1 & 425,0 & 0,1 & 0,2 & 0,1 & 0,058 & 0,6 & 2 & 0,1 & 2 \\
\hline
\end{tabular}

Tabela A.3: Dados do laboratório 4 


\begin{tabular}{|c|c|c|c|c|c|c|c|c|c|c|c|c|c|c|c|c|}
\hline \multicolumn{17}{|c|}{ Calibração } \\
\hline $\begin{array}{l}\text { F.M. } \\
\left({ }^{\circ} \mathrm{C}\right)\end{array}$ & \multicolumn{2}{|c|}{ A 1} & D & \multicolumn{2}{|c|}{ R 1} & $\mathrm{D}$ & \multicolumn{2}{|c|}{ A 2} & $\mathrm{D}$ & $\begin{array}{c}\text { med / } \\
\text { tend }\end{array}$ & $\begin{array}{l}\text { Desvio } \\
\text { Padrão }\end{array}$ & DPM & $\begin{array}{c}\mathrm{UE} \\
\mathrm{T}\end{array}$ & $\begin{array}{l}\mathrm{K} \\
\mathrm{T}\end{array}$ & $\begin{array}{l}\text { UE } \\
\text { Ind. }\end{array}$ & $\begin{array}{c}\mathrm{K} \\
\text { Ind. }\end{array}$ \\
\hline 25 & 19,6 & 17,9 & 1,7 & 24,5 & 23,0 & 1,5 & 24,5 & 23,0 & 1,5 & 1,6 & 0,115 & 0,067 & 0,1 & 2 & 0,1 & 2 \\
\hline 50 & 52,6 & 50,9 & 1,7 & 52,2 & 50,8 & 1,4 & 52,2 & 50,7 & 1,5 & 1,5 & 0,153 & 0,088 & 0,1 & 2 & 0,1 & 2 \\
\hline 175 & 176,8 & 177,8 & $-1,0$ & 175,1 & 176,1 & $-1,0$ & 174,4 & 175,2 & $-0,8$ & $-0,9$ & 0,115 & 0,067 & 0,2 & 2 & 0,1 & 2 \\
\hline 300 & 301,3 & 302,0 & $-0,7$ & 300,0 & 301,0 & $-1,0$ & 302,0 & 303,0 & $-1,0$ & $-0,9$ & 0,173 & 0,1 & 0,5 & 2 & 0,1 & 2 \\
\hline 425 & 426,0 & 426,4 & $-0,4$ & 423,3 & 424,1 & $-0,8$ & 428,5 & 428,6 & $-0,1$ & $-0,4$ & 0,351 & 0,203 & 0,5 & 2 & 0,1 & 2 \\
\hline
\end{tabular}

Tabela A.4: Dados do laboratório 5

\begin{tabular}{|c|c|c|c|c|c|c|c|c|c|c|c|c|c|c|c|c|}
\hline \multicolumn{17}{|c|}{ Calibração } \\
\hline $\begin{array}{l}\text { F.M. } \\
\left({ }^{\circ} \mathrm{C}\right)\end{array}$ & Art. & $\begin{array}{l}1 \\
\text { E.M.L. }\end{array}$ & $\mathrm{D}$ & Art. & $\begin{array}{l}1 \\
\text { E.M.L. }\end{array}$ & $\mathrm{D}$ & Art. & $\begin{array}{l} \\
\text { E.M.L. }\end{array}$ & $\mathrm{D}$ & $\begin{array}{c}\text { med / } \\
\text { tend }\end{array}$ & $\begin{array}{l}\text { Desvio } \\
\text { Padrão }\end{array}$ & DPM & $\begin{array}{c}\mathrm{UE} \\
\mathrm{T}\end{array}$ & $\begin{array}{l}\mathrm{K} \\
\mathrm{T}\end{array}$ & $\begin{array}{l}\text { UE } \\
\text { Ind. }\end{array}$ & $\begin{array}{c}\mathrm{K} \\
\text { Ind. }\end{array}$ \\
\hline 25 & 24,4 & 24,5 & $-0,1$ & 24,8 & 25,0 & $-0,2$ & 24,8 & 25,0 & $-0,2$ & $-0,2$ & 0,058 & 0,033 & & & 0,2 & 2 \\
\hline 50 & 50,7 & 50,6 & 0,1 & 50,8 & 50,5 & 0,3 & 50,5 & 50,3 & 0,2 & 0,2 & 0,1 & 0,058 & & & 0,2 & 2 \\
\hline 175 & 175,1 & 175,0 & 0,1 & 175,2 & 175,0 & 0,2 & 175,0 & 175,1 & $-0,1$ & 0,1 & 0,153 & 0,088 & & & 0,2 & 2 \\
\hline 300 & 301,0 & 301,4 & $-0,4$ & 301,3 & 301,3 & 0,0 & 300,9 & 301,7 & $-0,8$ & $-0,4$ & 0,4 & 0,231 & & & 0,2 & 2 \\
\hline 425 & 427,5 & 428,1 & $-0,6$ & 427,5 & 428,2 & $-0,7$ & 427,3 & 428,4 & $-1,1$ & $-0,8$ & 0,265 & 0,153 & & & 0,2 & 2 \\
\hline
\end{tabular}

Tabela A.5: Dados do laboratório 6

\begin{tabular}{|c|c|c|c|c|c|c|c|c|c|c|c|c|c|c|c|c|}
\hline \multicolumn{17}{|c|}{ Calibração } \\
\hline $\begin{array}{l}\text { F.M. } \\
\left({ }^{\circ} \mathrm{C}\right)\end{array}$ & \multicolumn{2}{|c|}{ A 1} & $\mathrm{D}$ & \multicolumn{2}{|c|}{ R 1} & D & \multicolumn{2}{|c|}{ A 2} & D & $\begin{array}{c}\text { med / } \\
\text { tend }\end{array}$ & $\begin{array}{c}\text { Desvio } \\
\text { Padrão }\end{array}$ & DPM & $\begin{array}{c}\text { UE } \\
\mathrm{T} \\
\end{array}$ & $\begin{array}{l}\mathrm{K} \\
\mathrm{T} \\
\end{array}$ & $\begin{array}{l}\mathrm{UE} \\
\text { Ind. }\end{array}$ & $\begin{array}{c}\mathrm{K} \\
\text { Ind. }\end{array}$ \\
\hline 25 & 24,4 & 24,5 & $-0,1$ & 24,8 & 25,0 & $-0,2$ & 24,8 & 25,0 & $-0,2$ & $-0,2$ & 0,058 & 0,033 & & & 0,2 & 2 \\
\hline 50 & 50,7 & 50,6 & 0,1 & 50,8 & 50,5 & 0,3 & 50,5 & 50,3 & 0,2 & 0,2 & 0,1 & 0,058 & & & 0,2 & 2 \\
\hline 175 & 175,1 & 175,0 & 0,1 & 175,2 & 175,0 & 0,2 & 175,0 & 175,1 & $-0,1$ & 0,1 & 0,153 & 0,088 & & & 0,2 & 2 \\
\hline 300 & 301,0 & 301,4 & $-0,4$ & 301,3 & 301,3 & 0,0 & 300,9 & 301,7 & $-0,8$ & $-0,4$ & 0,4 & 0,231 & & & 0,2 & 2 \\
\hline 425 & 427,5 & 428,1 & $-0,6$ & 427,5 & 428,2 & $-0,7$ & 427,3 & 428,4 & $-1,1$ & $-0,8$ & 0,265 & 0,153 & & & 0,2 & 2 \\
\hline
\end{tabular}

Tabela A.6: Dados do laboratório 7

\begin{tabular}{|c|c|c|c|c|c|c|c|c|c|c|c|c|c|c|c|c|}
\hline \multicolumn{17}{|c|}{ Calibração } \\
\hline \multirow{2}{*}{$\begin{array}{l}\text { F.M. } \\
\left({ }^{\circ} \mathrm{C}\right)\end{array}$} & \multicolumn{2}{|c|}{ A 1} & \multirow{2}{*}{$\mathrm{D}$} & \multicolumn{2}{|c|}{ R 1} & \multirow[t]{2}{*}{ D } & \multicolumn{2}{|c|}{ A 2} & \multirow{2}{*}{ D } & \multirow{2}{*}{$\begin{array}{c}\text { med / } \\
\text { tend }\end{array}$} & \multirow{2}{*}{$\begin{array}{l}\text { Desvio } \\
\text { Padrão }\end{array}$} & \multirow{2}{*}{ DPM } & \multirow{2}{*}{$\begin{array}{c}\text { UE } \\
\mathrm{T} \\
\end{array}$} & \multirow{2}{*}{$\begin{array}{l}\mathrm{K} \\
\mathrm{T}\end{array}$} & \multirow{2}{*}{$\begin{array}{l}\mathrm{UE} \\
\text { Ind. }\end{array}$} & $\begin{array}{c}\mathrm{K} \\
\text { Ind. }\end{array}$ \\
\hline & & & & & & & & & & & & & & & & \\
\hline 25 & 25,0 & 23,35 & 1,65 & 25,0 & 23,35 & 1,65 & 25,0 & 23,35 & 1,65 & 1,7 & 0,0 & 0,0 & 0,01 & 2 & 0,03 & 2 \\
\hline 50 & 50,0 & 48,47 & 1,53 & 50,0 & 48,47 & 1,53 & 50,0 & 48,44 & 1,56 & 1,5 & 0,017 & 0,01 & 0,01 & 2 & 0,03 & 2 \\
\hline 175 & 175,0 & 174,24 & 0,76 & 175,0 & 174,24 & 0,76 & 174,9 & 174,12 & 0,78 & 0,8 & 0,012 & 0,007 & 0,3 & 2 & 0,03 & 2 \\
\hline 300 & 300,0 & 300,1 & $-0,01$ & 300,2 & 300,21 & $-0,01$ & 300,3 & 300,21 & 0,09 & 0,0 & 0,058 & 0,033 & 0,6 & 2 & 0,03 & 2 \\
\hline 425 & 425,0 & 425,72 & $-0,72$ & 425,1 & 425,81 & $-0,71$ & 425,1 & 425,91 & $-0,81$ & $-0,7$ & 0,055 & 0,032 & 0,6 & 2 & 0,03 & 2 \\
\hline
\end{tabular}

Tabela A.7: Dados do laboratório 8

\begin{tabular}{|c|c|c|c|c|c|c|c|c|c|c|c|c|c|c|c|c|}
\hline \multicolumn{17}{|c|}{ Calibração } \\
\hline \multirow{2}{*}{$\begin{array}{l}\text { F.M. } \\
\left({ }^{\circ} \mathrm{C}\right)\end{array}$} & \multicolumn{2}{|c|}{ A 1} & \multirow[t]{2}{*}{ D } & \multicolumn{2}{|c|}{ R 1} & \multirow[t]{2}{*}{$\mathrm{D}$} & \multicolumn{2}{|c|}{ A 2} & \multirow[t]{2}{*}{$\mathrm{D}$} & \multirow{2}{*}{$\begin{array}{c}\text { med / } \\
\text { tend }\end{array}$} & \multirow{2}{*}{$\begin{array}{l}\text { Desvio } \\
\text { Padrão } \\
\end{array}$} & \multirow[t]{2}{*}{ DPM } & \multirow{2}{*}{$\begin{array}{l}\mathrm{UE} \\
\mathrm{T}\end{array}$} & \multirow{2}{*}{$\begin{array}{l}\mathrm{K} \\
\mathrm{T} \\
\end{array}$} & \multirow{2}{*}{$\begin{array}{l}\text { UE } \\
\text { Ind. }\end{array}$} & \multirow{2}{*}{$\begin{array}{r}\mathrm{K} \\
\text { Ind }\end{array}$} \\
\hline & Art. & E.M.L. & & Art. & E.M.L. & & Art. & E.M.L. & & & & & & & & \\
\hline 25 & 26,9 & 24,8 & 2,1 & 26,7 & 24,9 & 1,8 & 26,6 & 24,8 & 1,8 & 1,9 & 0,173 & 0,1 & 0,02 & 2 & 0,1 & 2 \\
\hline 50 & 51,4 & 49,5 & 1,9 & 51,8 & 50,1 & 1,7 & 51,5 & 49,7 & 1,8 & 1,8 & 0,1 & 0,058 & 0,03 & 2 & 0,1 & 2 \\
\hline 175 & 175,4 & 173,6 & 1,8 & 175,9 & 174,0 & 1,9 & 175,7 & 173,9 & 1,8 & 1,8 & 0,058 & 0,033 & 0,04 & 2 & 0,1 & 2 \\
\hline 300 & 300,8 & 298,6 & 2,2 & 300,7 & 298,6 & 2,1 & 300,6 & 298,5 & 2,1 & 2,1 & 0,058 & 0,033 & 0,04 & 2 & 0,1 & 2 \\
\hline 425 & 426,1 & 423,3 & 2,8 & 426,0 & 423,2 & 2,8 & 426,2 & 423,1 & 3,1 & 2,9 & 0,173 & 0,1 & 0,04 & 2 & 0,1 & 2 \\
\hline
\end{tabular}

Tabela A.8: Dados do laboratório 9 


\begin{tabular}{|c|c|c|c|c|c|c|c|c|c|c|c|c|c|c|c|c|}
\hline \multicolumn{17}{|c|}{ Calibração } \\
\hline $\begin{array}{l}\text { F.M. } \\
\left({ }^{\circ} \mathrm{C}\right)\end{array}$ & \multicolumn{2}{|c|}{ A 1} & $\mathrm{D}$ & \multicolumn{2}{|c|}{ R 1} & D & \multicolumn{2}{|c|}{ A 2} & D & $\begin{array}{c}\text { med / } \\
\text { tend }\end{array}$ & $\begin{array}{l}\text { Desvio } \\
\text { Padrão } \\
\end{array}$ & DPM & $\begin{array}{c}\text { UE } \\
\text { T }\end{array}$ & $\begin{array}{l}\mathrm{K} \\
\mathrm{T} \\
\end{array}$ & $\begin{array}{l}\text { UE } \\
\text { Ind. }\end{array}$ & $\begin{array}{c}\mathrm{K} \\
\text { Ind. }\end{array}$ \\
\hline 25 & 26,0 & 25,0 & 1,0 & 28,5 & 227,5 & 1,0 & 28,5 & 27,5 & 1,0 & 0,9 & 0,231 & 0,133 & 0,04 & 2 & 0,1 & 2 \\
\hline 50 & 51,3 & 50,3 & 1,0 & 51,0 & 50,1 & 0,9 & 51,1 & 50,3 & 0,8 & 0,3 & 0,987 & 0,57 & 0,04 & 2 & 0,1 & 2 \\
\hline 175 & 174,2 & 173,7 & 0,5 & 174,2 & 173,7 & 0,5 & 174,4 & 173,7 & 0,7 & 0,6 & 0,115 & 0,067 & 0,04 & 2 & 0,1 & 2 \\
\hline 300 & 296,4 & 295,5 & 0,9 & 293,0 & 293,8 & $-0,8$ & 293,3 & 294,1 & $-0,8$ & 0,3 & 0,981 & 0,567 & 1,5 & 2 & 0,3 & 2 \\
\hline 425 & 416,9 & 416,3 & 0,6 & 416,9 & 416,3 & 0,6 & 416,8 & 416,3 & 0,5 & 0,7 & 0,265 & 0,153 & 1,5 & 2 & 0,3 & 2 \\
\hline
\end{tabular}

Tabela A.9: Dados do laboratório 10

\begin{tabular}{|c|c|c|c|c|c|c|c|c|c|c|c|c|c|c|c|c|}
\hline \multicolumn{17}{|c|}{ Calibração } \\
\hline F.M. & \multicolumn{2}{|c|}{$\mathrm{A} 1$} & \multirow[t]{2}{*}{ D } & \multicolumn{2}{|c|}{ R 1} & \multirow[t]{2}{*}{ D } & \multicolumn{2}{|c|}{ A 2} & \multirow[t]{2}{*}{$\mathrm{D}$} & \multirow{2}{*}{$\begin{array}{c}\text { med / } \\
\text { tend }\end{array}$} & \multirow{2}{*}{$\begin{array}{l}\text { Desvio } \\
\text { Padrão }\end{array}$} & \multirow[t]{2}{*}{ DPM } & \multirow{2}{*}{$\begin{array}{c}\mathrm{UE} \\
\mathrm{T}\end{array}$} & \multirow{2}{*}{$\begin{array}{l}\mathrm{K} \\
\mathrm{T} \\
\end{array}$} & \multirow{2}{*}{$\begin{array}{l}\mathrm{UE} \\
\text { Ind. }\end{array}$} & \multirow{2}{*}{$\begin{array}{c}\mathrm{K} \\
\text { Ind. }\end{array}$} \\
\hline$\left({ }^{\circ} \mathrm{C}\right)$ & Art. & E.M.L. & & Art. & E.M.L. & & Art. & E.M.L. & & & & & & & & \\
\hline 25 & 21,1 & 19,35 & 1,75 & 22,9 & 21,3 & 1,6 & 22,9 & 21,3 & 1,6 & 1,7 & 0,087 & 0,05 & 0,07 & 2,04 & 0,06 & 2 \\
\hline 50 & 51,5 & 49,9 & 1,6 & 51,6 & 49,88 & 1,72 & 51,8 & 50,0 & 1,8 & 1,7 & 0,101 & 0,058 & 0,07 & 2,04 & 0,06 & 2 \\
\hline 175 & 176,3 & 174,85 & 1,45 & 176,5 & 174,97 & 1,53 & 176,6 & 175,0 & 1,6 & 1,5 & 0,075 & 0,043 & 0,09 & 2,04 & 0,06 & 2 \\
\hline 300 & 301,2 & 300,1 & 1,1 & 301,4 & 300,41 & 0,99 & 301,4 & 300,15 & 1,25 & 1,1 & 0,131 & 0,075 & 0,08 & 2,04 & 0,06 & 2 \\
\hline 425 & 426,4 & 425,8 & 0,6 & 426,4 & 425,84 & 0,56 & 426,4 & 425,24 & 1,16 & 0,8 & 0,335 & 0,194 & 0,08 & 2,04 & 0,06 & 2 \\
\hline
\end{tabular}

Tabela A.10: Dados do laboratório 11

Obs: A seqüência dos laboratórios das Tabelas 2.3 e A.1 a A.10 não está seguindo a mesma seqüência da Tabela 2.2, ou seja, por questão de ética, não vamos identificar as medições dos laboratórios. 


\section{Apêndice B}

\section{Tabelas de Incertezas dos Laboratórios}

\section{Laboratório 1}

\begin{tabular}{|c|c|c|c|c|c|c|}
\hline \multicolumn{7}{|c|}{ Cálculo de Incerteza: $50^{\circ} \mathrm{C}$} \\
\hline Fonte de Incerteza & Estimativa & Tipo & Distribuição & Divisor & Incerteza & G.L. \\
\hline Repetitividade & 0,186 & $\mathrm{~A}$ & Normal & 1 & 0,185592145 & 2 \\
\hline Resolução do Equipamento do lab. & 0,1 & $\mathrm{~B}$ & Retangular & 3,4641 & 0,028867527 & 30 \\
\hline Resolução do Artefato & 0,1 & $\mathrm{~B}$ & Retangular & 3,4641 & 0,028867527 & 30 \\
\hline Incerteza do Indicador (herdada) & 0,8 & $\mathrm{~B}$ & Normal & 2 & 0,4 & 30 \\
\hline Incerteza do Termopar (herdada) & 0,2 & $\mathrm{~B}$ & Normal & 2 & 0,1 & 30 \\
\hline Incerteza Combinada & \multicolumn{7}{|c|}{} & & 0,454 \\
\hline Graus de Liberdade Efetivos & \multicolumn{7}{|c|}{} & 34,820 \\
\hline Fator de Abrangência & \multicolumn{7}{|c|}{04920} \\
\hline Incerteza Expandida
\end{tabular}

Tabela B.1: Cálculo de incertezas para o laboratório 1 a $50^{\circ} \mathrm{C}$

\begin{tabular}{|c|c|c|c|c|c|c|}
\hline \multicolumn{7}{|c|}{ Cálculo de Incerteza: $175^{\circ} \mathrm{C}$} \\
\hline Fonte de Incerteza & Estimativa & Tipo & Distribuição & Divisor & Incerteza & G.L. \\
\hline Repetitividade & 0,176 & $\mathrm{~A}$ & Normal & 1 & 0,176383421 & 2 \\
\hline Resolução do Equipamento do lab. & 0,1 & $\mathrm{~B}$ & Retangular & 3,4641 & 0,028867527 & 30 \\
\hline Resolução do Artefato & 0,1 & $\mathrm{~B}$ & Retangular & 3,4641 & 0,028867527 & 30 \\
\hline Incerteza do Indicador (herdada) & 0,8 & $\mathrm{~B}$ & Normal & 2 & 0,4 & 30 \\
\hline Incerteza do Termopar (herdada) & 0,2 & $\mathrm{~B}$ & Normal & 2 & 0,1 & 30 \\
\hline Incerteza Combinada & \multicolumn{7}{|c|}{} & & 0,450 \\
\hline Graus de Liberdade Efetivos & \multicolumn{7}{|c|}{} & & 2,041 \\
\hline Fator de Abrangência & \multicolumn{7}{|c|}{0,920} \\
\hline Incerteza Expandida
\end{tabular}

Tabela B.2: Cálculo de incertezas para o laboratório 1 a $175^{\circ} \mathrm{C}$ 


\begin{tabular}{|c|c|c|c|c|c|c|}
\hline \multicolumn{7}{|c|}{ Cálculo de Incerteza: $300^{\circ} \mathrm{C}$} \\
\hline Fonte de Incerteza & Estimativa & Tipo & Distribuição & Divisor & Incerteza & G.L. \\
\hline Repetitividade & 0,306 & $\mathrm{~A}$ & Normal & 1 & 0,305505046 & 2 \\
\hline Resolução do Equipamento do lab. & 0,1 & $\mathrm{~B}$ & Retangular & 3,4641 & 0,028867527 & 30 \\
\hline Resolução do Artefato & 0,1 & $\mathrm{~B}$ & Retangular & 3,4641 & 0,028867527 & 30 \\
\hline Incerteza do Indicador (herdada) & 0,8 & $\mathrm{~B}$ & Normal & 2 & 0,4 & 30 \\
\hline Incerteza do Termopar (herdada) & 0,7 & $\mathrm{~B}$ & Normal & 2 & 0,35 & 30 \\
\hline Incerteza Combinada & \multicolumn{7}{|c|}{} & \multicolumn{3}{|r|}{24,9614} \\
\hline Graus de Liberdade Efetivos & \multicolumn{7}{|c|}{2,064} \\
\hline Fator de Abrangência & \multicolumn{7}{|c|}{1,268} \\
\hline Incerteza Expandida
\end{tabular}

Tabela B.3: Cálculo de incertezas para o laboratório 1 a $300^{\circ} \mathrm{C}$

\begin{tabular}{|c|c|c|c|c|c|c|}
\hline \multicolumn{7}{|c|}{ Cálculo de Incerteza: $425^{\circ} \mathrm{C}$} \\
\hline Fonte de Incerteza & Estimativa & Tipo & Distribuição & Divisor & Incerteza & G.L. \\
\hline Repetitividade & 0,033 & A & Normal & 1 & 0,033333333 & 2 \\
\hline Resolução do Equipamento do lab. & 0,1 & B & Retangular & 3,4641 & 0,028867527 & 30 \\
\hline Resolução do Artefato & 0,1 & $\mathrm{~B}$ & Retangular & 3,4641 & 0,028867527 & 30 \\
\hline Incerteza do Indicador (herdada) & 0,8 & $\mathrm{~B}$ & Normal & 2 & 0,4 & 30 \\
\hline Incerteza do Termopar (herdada) & 0,7 & $\mathrm{~B}$ & Normal & 2 & 0,35 & 30 \\
\hline Incerteza Combinada & & & & & & 0,534 \\
\hline Graus de Liberdade Efetivos & & & & & & 60,097 \\
\hline Fator de Abrangência & & & & & & 2,000 \\
\hline Incerteza Expandida & & & & & & 1,068 \\
\hline
\end{tabular}

Tabela B.4: Cálculo de incertezas para o laboratório 1 a $425^{\circ} \mathrm{C}$

\section{Laboratório 2}

\begin{tabular}{|c|c|c|c|c|c|c|}
\hline \multicolumn{7}{|c|}{ Cálculo de Incerteza: $25^{\circ} \mathrm{C}$} \\
\hline Fonte de Incerteza & Estimativa & Tipo & Distribuição & Divisor & Incerteza & G.L. \\
\hline Repetitividade & 0,033 & $\mathrm{~A}$ & Normal & 1 & 0,033333333 & 2 \\
\hline Resolução do Equipamento do lab. & 0,1 & $\mathrm{~B}$ & Retangular & 3,4641 & 0,028867527 & 30 \\
\hline Resolução do Artefato & 0,1 & $\mathrm{~B}$ & Retangular & 3,4641 & 0,028867527 & 30 \\
\hline Incerteza do Indicador (herdada) & 0,2 & $\mathrm{~B}$ & Normal & 2 & 0,1 & 30 \\
\hline Incerteza do Termopar (herdada) & 1,4 & $\mathrm{~B}$ & Normal & 2 & 0,7 & 30 \\
\hline Incerteza Combinada & \multicolumn{7}{|c|}{} & \multicolumn{4}{|r|}{31,569} \\
\hline Graus de Liberdade Efetivos & \multicolumn{7}{|c|}{2,040} \\
\hline Fator de Abrangência & \multicolumn{7}{|c|}{1,446} \\
\hline Incerteza Expandida
\end{tabular}

Tabela B.5: Cálculo de incertezas para o laboratório 2 a $25^{\circ} \mathrm{C}$

\begin{tabular}{|c|c|c|c|c|c|c|}
\hline \multicolumn{7}{|c|}{ Cálculo de Incerteza: $50^{\circ} \mathrm{C}$} \\
\hline Fonte de Incerteza & Estimativa & Tipo & Distribuição & Divisor & Incerteza & G.L. \\
\hline Repetitividade & 0,285 & $\mathrm{~A}$ & Normal & 1 & 0,284800125 & 2 \\
\hline Resolução do Equipamento do lab. & 0,1 & $\mathrm{~B}$ & Retangular & 3,4641 & 0,028867527 & 30 \\
\hline Resolução do Artefato & 0,1 & $\mathrm{~B}$ & Retangular & 3,4641 & 0,028867527 & 30 \\
\hline Incerteza do Indicador (herdada) & 0,2 & $\mathrm{~B}$ & Normal & 2 & 0,1 & 30 \\
\hline Incerteza do Termopar (herdada) & 1,4 & $\mathrm{~B}$ & Normal & 2 & 0,7 & 30 \\
\hline Incerteza Combinada & \multicolumn{7}{|c|}{0,763} \\
\hline Graus de Liberdade Efetivos & \multicolumn{7}{|c|}{30,066} \\
\hline Fator de Abrangência & \multicolumn{7}{|c|}{2,042} \\
\hline Incerteza Expandida & \multicolumn{7}{|c|}{1,559} \\
\hline
\end{tabular}

Tabela B.6: Cálculo de incertezas para o laboratório 2 a $50^{\circ} \mathrm{C}$ 


\begin{tabular}{|c|c|c|c|c|c|c|}
\hline \multicolumn{7}{|c|}{ Cálculo de Incerteza: $175^{\circ} \mathrm{C}$} \\
\hline Fonte de Incerteza & Estimativa & Tipo & Distribuição & Divisor & Incerteza & G.L. \\
\hline Repetitividade & 0,176 & A & Normal & 1 & 0,176383421 & 2 \\
\hline Resolução do Equipamento do lab. & 0,1 & B & Retangular & 3,4641 & 0,028867527 & 30 \\
\hline Resolução do Artefato & 0,1 & B & Retangular & 3,4641 & 0,028867527 & 30 \\
\hline Incerteza do Indicador (herdada) & 0,2 & $\mathrm{~B}$ & Normal & 2 & 0,1 & 30 \\
\hline Incerteza do Termopar (herdada) & 1,4 & B & Normal & 2 & 0,7 & 30 \\
\hline Incerteza Combinada & & & & & & 0,730 \\
\hline Graus de Liberdade Efetivos & & & & & & 33,431 \\
\hline Fator de Abrangência & & & & & & 2,035 \\
\hline Incerteza Expandida & & & & & & 1,485 \\
\hline
\end{tabular}

Tabela B.7: Cálculo de incertezas para o laboratório 2 a $175^{\circ} \mathrm{C}$

\begin{tabular}{|c|c|c|c|c|c|c|}
\hline \multicolumn{7}{|c|}{ Cálculo de Incerteza: $300^{\circ} \mathrm{C}$} \\
\hline Fonte de Incerteza & Estimativa & Tipo & Distribuição & Divisor & Incerteza & G.L. \\
\hline Repetitividade & 0,203 & $\mathrm{~A}$ & Normal & 1 & 0,202758751 & 2 \\
\hline Resolução do Equipamento do lab. & 0,1 & $\mathrm{~B}$ & Retangular & 3,4641 & 0,028867527 & 30 \\
\hline Resolução do Artefato & 0,1 & $\mathrm{~B}$ & Retangular & 3,4641 & 0,028867527 & 30 \\
\hline Incerteza do Indicador (herdada) & 0,2 & $\mathrm{~B}$ & Normal & 2 & 0,1 & 30 \\
\hline Incerteza do Termopar (herdada) & 1,4 & B & Normal & 2 & 0,7 & 30 \\
\hline Incerteza Combinada & & & & & & 0,737 \\
\hline Graus de Liberdade Efetivos & & & & & & 33,282 \\
\hline Fator de Abrangência & & & & & & 2,035 \\
\hline Incerteza Expandida & & & & & & 1,499 \\
\hline
\end{tabular}

Tabela B.8: Cálculo de incertezas para o laboratório 2 a $300^{\circ} \mathrm{C}$

\begin{tabular}{|c|c|c|c|c|c|c|}
\hline \multicolumn{7}{|c|}{ Cálculo de Incerteza: $425^{\circ} \mathrm{C}$} \\
\hline Fonte de Incerteza & Estimativa & Tipo & Distribuição & Divisor & Incerteza & G.L. \\
\hline Repetitividade & 0,186 & A & Normal & 1 & 0,185592145 & 2 \\
\hline Resolução do Equipamento do lab. & 0,1 & B & Retangular & 3,4641 & 0,028867527 & 30 \\
\hline Resolução do Artefato & 0,1 & B & Retangular & 3,4641 & 0,028867527 & 30 \\
\hline Incerteza do Indicador (herdada) & 0,2 & B & Normal & 2 & 0,1 & 30 \\
\hline Incerteza do Termopar (herdada) & 2,4 & $\mathrm{~B}$ & Normal & 2 & 1,2 & 30 \\
\hline Incerteza Combinada & & & & & & 1,219 \\
\hline Graus de Liberdade Efetivos & & & & & & 31,679 \\
\hline Fator de Abrangência & & & & & & 2,040 \\
\hline Incerteza Expandida & & & & & & 2,486 \\
\hline
\end{tabular}

Tabela B.9: Cálculo de incertezas para o laboratório 2 a $425^{\circ} \mathrm{C}$

\section{Laboratório 3}

\begin{tabular}{|c|c|c|c|c|c|c|}
\hline \multicolumn{7}{|c|}{ Cálculo de Incerteza: $25^{\circ} \mathrm{C}$} \\
\hline Fonte de Incerteza & Estimativa & Tipo & Distribuição & Divisor & Incerteza & G.L. \\
\hline Repetitividade & 0,145 & $\mathrm{~A}$ & Normal & 1 & 0,145296631 & 2 \\
\hline Resolução do Equipamento do lab. & 0,1 & $\bar{B}$ & Retangular & 3,4641 & 0,028867527 & 30 \\
\hline Resolução do Artefato & 0,1 & $\mathrm{~B}$ & Retangular & 3,4641 & 0,028867527 & 30 \\
\hline Incerteza do Indicador (herdada) & 0,1 & B & Normal & 2 & 0,05 & 30 \\
\hline Incerteza do Termopar (herdada) & 0,3 & B & Normal & 2 & 0,15 & 30 \\
\hline Incerteza Combinada & & & & & & 0,219 \\
\hline Graus de Liberdade Efetivos & & & & & & 9,513 \\
\hline Fator de Abrangência & & & & & & 2,262 \\
\hline Incerteza Expandida & & & & & & 0,494 \\
\hline
\end{tabular}

Tabela B.10: Cálculo de incertezas para o laboratório 3 a $25^{\circ} \mathrm{C}$ 
Cálculo de Incerteza: $50^{\circ} \mathrm{C}$

\begin{tabular}{|c|c|c|c|c|c|c|}
\hline Fonte de Incerteza & Estimativa & Tipo & Distribuição & Divisor & Incerteza & G.L. \\
\hline Repetitividade & 0,088 & $\mathrm{~A}$ & Normal & 1 & 0,08819171 & 2 \\
\hline Resolução do Equipamento do lab. & 0,1 & $\mathrm{~B}$ & Retangular & 3,4641 & 0,028867527 & 30 \\
\hline Resolução do Artefato & 0,1 & B & Retangular & 3,4641 & 0,028867527 & 30 \\
\hline Incerteza do Indicador (herdada) & 0,1 & B & Normal & 2 & 0,05 & 30 \\
\hline Incerteza do Termopar (herdada) & 0,3 & $\mathrm{~B}$ & Normal & 2 & 0,15 & 30 \\
\hline Incerteza Combinada & & & & & & 0,186 \\
\hline Graus de Liberdade Efetivos & & & & & & 25,042 \\
\hline Fator de Abrangência & & & & & & 2,060 \\
\hline Incerteza Expandida & & & & & & 0,382 \\
\hline
\end{tabular}

Tabela B.11: Cálculo de incertezas para o laboratório 3 a $50^{\circ} \mathrm{C}$

\begin{tabular}{|c|c|c|c|c|c|c|}
\hline \multicolumn{7}{|c|}{ Cálculo de Incerteza: $175^{\circ} \mathrm{C}$} \\
\hline Fonte de Incerteza & Estimativa & Tipo & Distribuição & Divisor & Incerteza & G.L. \\
\hline Repetitividade & 0,433 & A & Normal & 1 & 0,433333333 & 2 \\
\hline Resolução do Equipamento do lab. & 0,1 & $\mathrm{~B}$ & Retangular & 3,4641 & 0,028867527 & 30 \\
\hline Resolução do Artefato & 0,1 & $\mathrm{~B}$ & Retangular & 3,4641 & 0,028867527 & 30 \\
\hline Incerteza do Indicador (herdada) & 0,1 & B & Normal & 2 & 0,05 & 30 \\
\hline Incerteza do Termopar (herdada) & 0,3 & $\mathrm{~B}$ & Normal & 2 & 0,15 & 30 \\
\hline Incerteza Combinada & & & & & & 0,463 \\
\hline Graus de Liberdade Efetivos & & & & & & 2,606 \\
\hline Fator de Abrangência & & & & & & 4,303 \\
\hline Incerteza Expandida & & & & & & 1,992 \\
\hline
\end{tabular}

Tabela B.12: Cálculo de incertezas para o laboratório 3 a $175^{\circ} \mathrm{C}$

\begin{tabular}{|c|c|c|c|c|c|c|}
\hline \multicolumn{7}{|c|}{ Cálculo de Incerteza: $300^{\circ} \mathrm{C}$} \\
\hline Fonte de Incerteza & Estimativa & Tipo & Distribuição & Divisor & Incerteza & G.L. \\
\hline Repetitividade & 0,351 & $\mathrm{~A}$ & Normal & 1 & 0,351188458 & 2 \\
\hline Resolução do Equipamento do lab. & 0,1 & B & Retangular & 3,4641 & 0,028867527 & 30 \\
\hline Resolução do Artefato & 0,1 & $\mathrm{~B}$ & Retangular & 3,4641 & 0,028867527 & 30 \\
\hline Incerteza do Indicador (herdada) & 0,1 & B & Normal & 2 & 0,05 & 30 \\
\hline Incerteza do Termopar (herdada) & 0,3 & $\mathrm{~B}$ & Normal & 2 & 0,15 & 30 \\
\hline Incerteza Combinada & & & & & & 0,387 \\
\hline Graus de Liberdade Efetivos & & & & & & 2,952 \\
\hline Fator de Abrangência & & & & & & 4,303 \\
\hline Incerteza Expandida & & & & & & 1,688 \\
\hline
\end{tabular}

Tabela B.13: Cálculo de incertezas para o laboratório 3 a $300^{\circ} \mathrm{C}$

\begin{tabular}{|c|c|c|c|c|c|c|}
\hline \multicolumn{7}{|c|}{ Cálculo de Incerteza: $425^{\circ} \mathrm{C}$} \\
\hline Fonte de Incerteza & Estimativa & Tipo & Distribuição & Divisor & Incerteza & G.L. \\
\hline Repetitividade & 0,0 & A & Normal & 1 & 0,0 & 2 \\
\hline Resolução do Equipamento do lab. & 0,1 & B & Retangular & 3,4641 & 0,028867527 & 30 \\
\hline Resolução do Artefato & 0,1 & $\mathrm{~B}$ & Retangular & 3,4641 & 0,028867527 & 30 \\
\hline Incerteza do Indicador (herdada) & 0,1 & $\mathrm{~B}$ & Normal & 2 & 0,05 & 30 \\
\hline Incerteza do Termopar (herdada) & 0,6 & B & Normal & 2 & 0,3 & 30 \\
\hline Incerteza Combinada & & & & & & 0,317 \\
\hline Graus de Liberdade Efetivos & & & & & & 32,811 \\
\hline Fator de Abrangência & & & & & & 2,037 \\
\hline Incerteza Expandida & & & & & & 0,625 \\
\hline
\end{tabular}

Tabela B.14: Cálculo de incertezas para o laboratório 3 a $425^{\circ} \mathrm{C}$ 


\section{Laboratório 4}

\begin{tabular}{|c|c|c|c|c|c|c|}
\hline \multicolumn{7}{|c|}{ Cálculo de Incerteza: $25^{\circ} \mathrm{C}$} \\
\hline Fonte de Incerteza & Estimativa & Tipo & Distribuição & Divisor & Incerteza & G.L. \\
\hline Repetitividade & 0,0 & A & Normal & 1 & $1,72064 e^{-8}$ & 2 \\
\hline Resolução do Equipamento do lab. & 0,1 & B & Retangular & 3,4641 & 0,028867527 & 30 \\
\hline Resolução do Artefato & 0,1 & B & Retangular & 3,4641 & 0,028867527 & 30 \\
\hline Incerteza do Indicador (herdada) & 0,1 & $\mathrm{~B}$ & Normal & 2 & 0,05 & 30 \\
\hline Incerteza do Termopar (herdada) & 0,2 & B & Normal & 2 & 0,1 & 30 \\
\hline Incerteza Combinada & & & & & & 0,119 \\
\hline Graus de Liberdade Efetivos & & & & & & 65,953 \\
\hline Fator de Abrangência & & & & & & 2,004 \\
\hline Incerteza Expandida & & & & & & 0,239 \\
\hline
\end{tabular}

Tabela B.15: Cálculo de incertezas para o laboratório 4 a $25^{\circ} \mathrm{C}$

\begin{tabular}{|c|c|c|c|c|c|c|}
\hline \multicolumn{7}{|c|}{ Cálculo de Incerteza: $50^{\circ} \mathrm{C}$} \\
\hline Fonte de Incerteza & Estimativa & Tipo & Distribuição & Divisor & Incerteza & G.L. \\
\hline Repetitividade & 0,033 & A & Normal & 1 & 0,033333333 & 2 \\
\hline Resolução do Equipamento do lab. & 0,1 & $\mathrm{~B}$ & Retangular & 3,4641 & 0,028867527 & 30 \\
\hline Resolução do Artefato & 0,1 & B & Retangular & 3,4641 & 0,028867527 & 30 \\
\hline Incerteza do Indicador (herdada) & 0,1 & B & Normal & 2 & 0,05 & 30 \\
\hline Incerteza do Termopar (herdada) & 0,2 & B & Normal & 2 & 0,1 & 30 \\
\hline Incerteza Combinada & & & & & & 0,124 \\
\hline Graus de Liberdade Efetivos & & & & & & 55,505 \\
\hline Fator de Abrangência & & & & & & 2,004 \\
\hline Incerteza Expandida & & & & & & 0,248 \\
\hline
\end{tabular}

Tabela B.16: Cálculo de incertezas para o laboratório 4 a $50^{\circ} \mathrm{C}$

\begin{tabular}{|c|c|c|c|c|c|c|}
\hline \multicolumn{7}{|c|}{ Cálculo de Incerteza: $175^{\circ} \mathrm{C}$} \\
\hline Fonte de Incerteza & Estimativa & Tipo & Distribuição & Divisor & Incerteza & G.L. \\
\hline Repetitividade & 0,067 & A & Normal & 1 & 0,066666667 & 2 \\
\hline Resolução do Equipamento do lab. & 0,1 & B & Retangular & 3,4641 & 0,028867527 & 30 \\
\hline Resolução do Artefato & 0,1 & B & Retangular & 3,4641 & 0,028867527 & 30 \\
\hline Incerteza do Indicador (herdada) & 0,1 & $\mathrm{~B}$ & Normal & 2 & 0,05 & 30 \\
\hline Incerteza do Termopar (herdada) & 0,2 & B & Normal & 2 & 0,1 & 30 \\
\hline Incerteza Combinada & & & & & & 0,136 \\
\hline Graus de Liberdade Efetivos & & & & & & 25,725 \\
\hline Fator de Abrangência & & & & & & 2,060 \\
\hline Incerteza Expandida & & & & & & 0,281 \\
\hline
\end{tabular}

Tabela B.17: Cálculo de incertezas para o laboratório 4 a $175^{\circ} \mathrm{C}$ 


\begin{tabular}{|c|c|c|c|c|c|c|}
\hline \multicolumn{7}{|c|}{ Cálculo de Incerteza: $300^{\circ} \mathrm{C}$} \\
\hline Fonte de Incerteza & Estimativa & Tipo & Distribuição & Divisor & Incerteza & G.L. \\
\hline Repetitividade & 0,120 & A & Normal & 1 & 0,120185043 & 2 \\
\hline Resolução do Equipamento do lab. & 0,1 & B & Retangular & 3,4641 & 0,028867527 & 30 \\
\hline Resolução do Artefato & 0,1 & B & Retangular & 3,4641 & 0,028867527 & 30 \\
\hline Incerteza do Indicador (herdada) & 0,1 & $\mathrm{~B}$ & Normal & 2 & 0,05 & 30 \\
\hline Incerteza do Termopar (herdada) & 0,6 & $\mathrm{~B}$ & Normal & 2 & 0,3 & 30 \\
\hline Incerteza Combinada & & & & & & 0,330 \\
\hline Graus de Liberdade Efetivos & & & & & & 31,493 \\
\hline Fator de Abrangência & & & & & & 2,040 \\
\hline Incerteza Expandida & & & & & & 0,672 \\
\hline
\end{tabular}

Tabela B.18: Cálculo de incertezas para o laboratório 4 a $300^{\circ} \mathrm{C}$

\begin{tabular}{|c|c|c|c|c|c|c|}
\hline \multicolumn{7}{|c|}{ Cálculo de Incerteza: $425^{\circ} \mathrm{C}$} \\
\hline Fonte de Incerteza & Estimativa & Tipo & Distribuição & Divisor & Incerteza & G.L. \\
\hline Repetitividade & 0,058 & A & Normal & 1 & 0,057735027 & 2 \\
\hline Resolução do Equipamento do lab. & 0,1 & B & Retangular & 3,4641 & 0,028867527 & 30 \\
\hline Resolução do Artefato & 0,1 & B & Retangular & 3,4641 & 0,028867527 & 30 \\
\hline Incerteza do Indicador (herdada) & 0,1 & B & Normal & 2 & 0,05 & 30 \\
\hline Incerteza do Termopar (herdada) & 0,6 & $\mathrm{~B}$ & Normal & 2 & 0,3 & 30 \\
\hline Incerteza Combinada & & & & & & 0,312 \\
\hline Graus de Liberdade Efetivos & & & & & & 34,467 \\
\hline Fator de Abrangência & & & & & & 2,032 \\
\hline Incerteza Expandida & & & & & & 0,635 \\
\hline
\end{tabular}

Tabela B.19: Cálculo de incertezas para o laboratório 4 a $425^{\circ} \mathrm{C}$

\section{Laboratório 5}

\begin{tabular}{|c|c|c|c|c|c|c|}
\hline \multicolumn{7}{|c|}{ Cálculo de Incerteza: $25^{\circ} \mathrm{C}$} \\
\hline Fonte de Incerteza & Estimativa & Tipo & Distribuição & Divisor & Incerteza & G.L. \\
\hline Repetitividade & 0,067 & A & Normal & 1 & 0,06666667 & 2 \\
\hline Resolução do Equipamento do lab. & 0,1 & B & Retangular & 3,4641 & 0,028867527 & 30 \\
\hline Resolução do Artefato & 0,1 & $\mathrm{~B}$ & Retangular & 3,4641 & 0,028867527 & 30 \\
\hline Incerteza do Indicador (herdada) & 0,1 & $\mathrm{~B}$ & Normal & 2 & 0,05 & 30 \\
\hline Incerteza do Termopar (herdada) & 0,1 & B & Normal & 2 & 0,05 & 30 \\
\hline Incerteza Combinada & & & & & & 0,105 \\
\hline Graus de Liberdade Efetivos & & & & & & 11,940 \\
\hline Fator de Abrangência & & & & & & 2,201 \\
\hline Incerteza Expandida & & & & & & 0,232 \\
\hline
\end{tabular}

Tabela B.20: Cálculo de incertezas para o laboratório 5 a $25^{\circ} \mathrm{C}$

\begin{tabular}{|c|c|c|c|c|c|c|}
\hline \multicolumn{7}{|c|}{ Cálculo de Incerteza: $50^{\circ} \mathrm{C}$} \\
\hline Fonte de Incerteza & Estimativa & Tipo & Distribuição & Divisor & Incerteza & G.L. \\
\hline Repetitividade & 0,088 & A & Normal & 1 & 0,08819171 & 2 \\
\hline Resolução do Equipamento do lab. & 0,1 & B & Retangular & 3,4641 & 0,028867527 & 30 \\
\hline Resolução do Artefato & 0,1 & $\bar{B}$ & Retangular & 3,4641 & 0,028867527 & 30 \\
\hline Incerteza do Indicador (herdada) & 0,1 & $\bar{B}$ & Normal & 2 & 0,05 & 30 \\
\hline Incerteza do Termopar (herdada) & 0,1 & $\mathrm{~B}$ & Normal & 2 & 0,05 & 30 \\
\hline Incerteza Combinada & & & & & & 0,120 \\
\hline Graus de Liberdade Efetivos & & & & & & 6,794 \\
\hline Fator de Abrangência & & & & & & 2,060 \\
\hline Incerteza Expandida & & & & & & 0,294 \\
\hline
\end{tabular}

Tabela B.21: Cálculo de incertezas para o laboratório 5 a $50^{\circ} \mathrm{C}$ 


\begin{tabular}{|c|c|c|c|c|c|c|}
\hline \multicolumn{7}{|c|}{ Cálculo de Incerteza: $175^{\circ} \mathrm{C}$} \\
\hline Fonte de Incerteza & Estimativa & Tipo & Distribuição & Divisor & Incerteza & G.L. \\
\hline Repetitividade & 0,067 & A & Normal & 1 & 0,06666667 & 2 \\
\hline Resolução do Equipamento do lab. & 0,1 & B & Retangular & 3,4641 & 0,028867527 & 30 \\
\hline Resolução do Artefato & 0,1 & B & Retangular & 3,4641 & 0,028867527 & 30 \\
\hline Incerteza do Indicador (herdada) & 0,1 & $\mathrm{~B}$ & Normal & 2 & 0,05 & 30 \\
\hline Incerteza do Termopar (herdada) & 0,2 & B & Normal & 2 & 0,1 & 30 \\
\hline Incerteza Combinada & & & & & & 0,136 \\
\hline Graus de Liberdade Efetivos & & & & & & 25,725 \\
\hline Fator de Abrangência & & & & & & 2,060 \\
\hline Incerteza Expandida & & & & & & 0,281 \\
\hline
\end{tabular}

Tabela B.22: Cálculo de incertezas para o laboratório 5 a $175^{\circ} \mathrm{C}$

\begin{tabular}{|c|c|c|c|c|c|c|}
\hline \multicolumn{7}{|c|}{ Cálculo de Incerteza: $300^{\circ} \mathrm{C}$} \\
\hline Fonte de Incerteza & Estimativa & Tipo & Distribuição & Divisor & Incerteza & G.L. \\
\hline Repetitividade & 0,1 & $\mathrm{~A}$ & Normal & 1 & 0,1 & 2 \\
\hline Resolução do Equipamento do lab. & 0,1 & $\bar{B}$ & Retangular & 3,4641 & 0,028867527 & 30 \\
\hline Resolução do Artefato & 0,1 & $\mathrm{~B}$ & Retangular & 3,4641 & 0,028867527 & 30 \\
\hline Incerteza do Indicador (herdada) & 0,1 & $\mathrm{~B}$ & Normal & 2 & 0,05 & 30 \\
\hline Incerteza do Termopar (herdada) & 0,5 & B & Normal & 2 & 0,25 & 30 \\
\hline Incerteza Combinada & & & & & & 0,277 \\
\hline Graus de Liberdade Efetivos & & & & & & 32,571 \\
\hline Fator de Abrangência & & & & & & 2,037 \\
\hline Incerteza Expandida & & & & & & 0,564 \\
\hline
\end{tabular}

Tabela B.23: Cálculo de incertezas para o laboratório 5 a $300^{\circ} \mathrm{C}$

\begin{tabular}{|c|c|c|c|c|c|c|}
\hline \multicolumn{7}{|c|}{ Cálculo de Incerteza: $425^{\circ} \mathrm{C}$} \\
\hline Fonte de Incerteza & Estimativa & Tipo & Distribuição & Divisor & Incerteza & G.L. \\
\hline Repetitividade & 0,203 & A & Normal & 1 & 0,202758751 & 2 \\
\hline Resolução do Equipamento do lab. & 0,1 & B & Retangular & 3,4641 & 0,028867527 & 30 \\
\hline Resolução do Artefato & 0,1 & B & Retangular & 3,4641 & 0,028867527 & 30 \\
\hline Incerteza do Indicador (herdada) & 0,1 & B & Normal & 2 & 0,05 & 30 \\
\hline Incerteza do Termopar (herdada) & 0,5 & $\mathrm{~B}$ & Normal & 2 & 0,25 & 30 \\
\hline Incerteza Combinada & & & & & & 0,328 \\
\hline Graus de Liberdade Efetivos & & & & & & 11,907 \\
\hline Fator de Abrangência & & & & & & 2,201 \\
\hline Incerteza Expandida & & & & & & 0,723 \\
\hline
\end{tabular}

Tabela B.24: Cálculo de incertezas para o laboratório 5 a $425^{\circ} \mathrm{C}$

\section{Laboratório 6}

\begin{tabular}{|c|c|c|c|c|c|c|}
\hline \multicolumn{7}{|c|}{ Cálculo de Incerteza: $25^{\circ} \mathrm{C}$} \\
\hline Fonte de Incerteza & Estimativa & Tipo & Distribuição & Divisor & Incerteza & G.L. \\
\hline Repetitividade & 0,033 & $\mathrm{~A}$ & Normal & 1 & 0,033333333 & 2 \\
\hline Resolução do Equipamento do lab. & 0,1 & $\bar{B}$ & Retangular & 3,4641 & 0,028867527 & 30 \\
\hline Resolução do Artefato & 0,1 & $\mathrm{~B}$ & Retangular & 3,4641 & 0,028867527 & 30 \\
\hline Incerteza do Indicador (herdada) & 0,2 & B & Normal & 2 & 0,1 & 30 \\
\hline Incerteza do Termopar (herdada) & 0,0 & B & Normal & 2 & 0,0 & 30 \\
\hline Incerteza Combinada & & & & & & 0,113 \\
\hline Graus de Liberdade Efetivos & & & & & & 40,849 \\
\hline Fator de Abrangência & & & & & & 2,021 \\
\hline Incerteza Expandida & & & & & & 0,228 \\
\hline
\end{tabular}

Tabela B.25: Cálculo de incertezas para o laboratório 6 a $25^{\circ} \mathrm{C}$ 
Cálculo de Incerteza: $50^{\circ} \mathrm{C}$

\begin{tabular}{|c|c|c|c|c|c|c|}
\hline \multicolumn{7}{|c|}{ Cálculo de Incerteza: $50^{\circ} \mathrm{C}$} \\
\hline Fonte de Incerteza & Estimativa & Tipo & Distribuição & Divisor & Incerteza & G.L. \\
\hline Repetitividade & 0,058 & A & Normal & 1 & 0,057735027 & 2 \\
\hline Resolução do Equipamento do lab. & 0,1 & $\mathrm{~B}$ & Retangular & 3,4641 & 0,028867527 & 30 \\
\hline Resolução do Artefato & 0,1 & $\mathrm{~B}$ & Retangular & 3,4641 & 0,028867527 & 30 \\
\hline Incerteza do Indicador (herdada) & 0,2 & $\mathrm{~B}$ & Normal & 2 & 0,1 & 30 \\
\hline Incerteza do Termopar (herdada) & 0,0 & $\mathrm{~B}$ & Normal & 2 & 0,0 & 30 \\
\hline Incerteza Combinada & & & & & & 0,122 \\
\hline Graus de Liberdade Efetivos & & & & & & 25,181 \\
\hline Fator de Abrangência & & & & & & 2,060 \\
\hline Incerteza Expandida & & & & & & 0,252 \\
\hline
\end{tabular}

Tabela B.26: Cálculo de incertezas para o laboratório 6 a $50^{\circ} \mathrm{C}$

\begin{tabular}{|c|c|c|c|c|c|c|}
\hline \multicolumn{7}{|c|}{ Cálculo de Incerteza: $175^{\circ} \mathrm{C}$} \\
\hline Fonte de Incerteza & Estimativa & Tipo & Distribuição & Divisor & Incerteza & G.L. \\
\hline Repetitividade & 0,088 & A & Normal & 1 & 0,08819171 & 2 \\
\hline Resolução do Equipamento do lab. & 0,1 & $\mathrm{~B}$ & Retangular & 3,4641 & 0,028867527 & 30 \\
\hline Resolução do Artefato & 0,1 & $\mathrm{~B}$ & Retangular & 3,4641 & 0,028867527 & 30 \\
\hline Incerteza do Indicador (herdada) & 0,2 & B & Normal & 2 & 0,1 & 30 \\
\hline Incerteza do Termopar (herdada) & 0,0 & $\mathrm{~B}$ & Normal & 2 & 0,0 & 30 \\
\hline Incerteza Combinada & & & & & & 0,139 \\
\hline Graus de Liberdade Efetivos & & & & & & 11,244 \\
\hline Fator de Abrangência & & & & & & 2,201 \\
\hline Incerteza Expandida & & & & & & 0,307 \\
\hline
\end{tabular}

Tabela B.27: Cálculo de incertezas para o laboratório 6 a $175^{\circ} \mathrm{C}$

\begin{tabular}{|c|c|c|c|c|c|c|}
\hline \multicolumn{7}{|c|}{ Cálculo de Incerteza: $300^{\circ} \mathrm{C}$} \\
\hline Fonte de Incerteza & Estimativa & Tipo & Distribuição & Divisor & Incerteza & G.L. \\
\hline Repetitividade & 0,231 & $\mathrm{~A}$ & Normal & 1 & 0,230940108 & 2 \\
\hline Resolução do Equipamento do lab. & 0,1 & B & Retangular & 3,4641 & 0,028867527 & 30 \\
\hline Resolução do Artefato & 0,1 & $\mathrm{~B}$ & Retangular & 3,4641 & 0,028867527 & 30 \\
\hline Incerteza do Indicador (herdada) & 0,2 & B & Normal & 2 & 0,1 & 30 \\
\hline Incerteza do Termopar (herdada) & 0,0 & $\mathrm{~B}$ & Normal & 2 & 0,0 & 30 \\
\hline Incerteza Combinada & & & & & & 0,255 \\
\hline Graus de Liberdade Efetivos & & & & & & 2,964 \\
\hline Fator de Abrangência & & & & & & 4,303 \\
\hline Incerteza Expandida & & & & & & 1,097 \\
\hline
\end{tabular}

Tabela B.28: Cálculo de incertezas para o laboratório 6 a $300^{\circ} \mathrm{C}$

\begin{tabular}{|c|c|c|c|c|c|c|}
\hline \multicolumn{7}{|c|}{ Cálculo de Incerteza: $425^{\circ} \mathrm{C}$} \\
\hline Fonte de Incerteza & Estimativa & Tipo & Distribuição & Divisor & Incerteza & G.L. \\
\hline Repetitividade & 0,153 & A & Normal & 1 & 0,152752523 & 2 \\
\hline Resolução do Equipamento do lab. & 0,1 & $\mathrm{~B}$ & Retangular & 3,4641 & 0,028867527 & 30 \\
\hline Resolução do Artefato & 0,1 & B & Retangular & 3,4641 & 0,028867527 & 30 \\
\hline Incerteza do Indicador (herdada) & 0,2 & $\mathrm{~B}$ & Normal & 2 & 0,1 & 30 \\
\hline Incerteza do Termopar (herdada) & 0,0 & B & Normal & 2 & 0,0 & 30 \\
\hline Incerteza Combinada & & & & & & 0,187 \\
\hline Graus de Liberdade Efetivos & & & & & & 4,445 \\
\hline Fator de Abrangência & & & & & & 2,776 \\
\hline Incerteza Expandida & & & & & & 0,519 \\
\hline
\end{tabular}

Tabela B.29: Cálculo de incertezas para o laboratório 6 a $425^{\circ} \mathrm{C}$ 


\section{Laboratório 7}

\begin{tabular}{|c|c|c|c|c|c|c|}
\hline \multicolumn{7}{|c|}{ Cálculo de Incerteza: $25^{\circ} \mathrm{C}$} \\
\hline Fonte de Incerteza & Estimativa & Tipo & Distribuição & Divisor & Incerteza & G.L. \\
\hline Repetitividade & 0,033 & A & Normal & 1 & 0,033333333 & 2 \\
\hline Resolução do Equipamento do lab. & 0,1 & B & Retangular & 3,4641 & 0,028867527 & 30 \\
\hline Resolução do Artefato & 0,1 & B & Retangular & 3,4641 & 0,028867527 & 30 \\
\hline Incerteza do Indicador (herdada) & 0,2 & $\mathrm{~B}$ & Normal & 2 & 0,1 & 30 \\
\hline Incerteza do Termopar (herdada) & 0,0 & B & Normal & 2 & 0,0 & 30 \\
\hline Incerteza Combinada & & & & & & 0,113 \\
\hline Graus de Liberdade Efetivos & & & & & & 40,849 \\
\hline Fator de Abrangência & & & & & & 2,021 \\
\hline Incerteza Expandida & & & & & & 0,228 \\
\hline
\end{tabular}

Tabela B.30: Cálculo de incertezas para o laboratório 7 a $25^{\circ} \mathrm{C}$

\begin{tabular}{|c|c|c|c|c|c|c|}
\hline \multicolumn{7}{|c|}{ Cálculo de Incerteza: $50^{\circ} \mathrm{C}$} \\
\hline Fonte de Incerteza & Estimativa & Tipo & Distribuição & Divisor & Incerteza & G.L. \\
\hline Repetitividade & 0,058 & A & Normal & 1 & 0,057735027 & 2 \\
\hline Resolução do Equipamento do lab. & 0,1 & $\mathrm{~B}$ & Retangular & 3,4641 & 0,028867527 & 30 \\
\hline Resolução do Artefato & 0,1 & B & Retangular & 3,4641 & 0,028867527 & 30 \\
\hline Incerteza do Indicador (herdada) & 0,2 & B & Normal & 2 & 0,1 & 30 \\
\hline Incerteza do Termopar (herdada) & 0,0 & B & Normal & 2 & 0,0 & 30 \\
\hline Incerteza Combinada & & & & & & 0,122 \\
\hline Graus de Liberdade Efetivos & & & & & & 25,181 \\
\hline Fator de Abrangência & & & & & & 2,060 \\
\hline Incerteza Expandida & & & & & & 0,252 \\
\hline
\end{tabular}

Tabela B.31: Cálculo de incertezas para o laboratório 7 a $50^{\circ} \mathrm{C}$

\begin{tabular}{|c|c|c|c|c|c|c|}
\hline \multicolumn{7}{|c|}{ Cálculo de Incerteza: $175^{\circ} \mathrm{C}$} \\
\hline Fonte de Incerteza & Estimativa & Tipo & Distribuição & Divisor & Incerteza & G.L. \\
\hline Repetitividade & 0,088 & A & Normal & 1 & 0,08819171 & 2 \\
\hline Resolução do Equipamento do lab. & 0,1 & B & Retangular & 3,4641 & 0,028867527 & 30 \\
\hline Resolução do Artefato & 0,1 & B & Retangular & 3,4641 & 0,028867527 & 30 \\
\hline Incerteza do Indicador (herdada) & 0,2 & B & Normal & 2 & 0,1 & 30 \\
\hline Incerteza do Termopar (herdada) & 0,0 & B & Normal & 2 & 0,0 & 30 \\
\hline Incerteza Combinada & & & & & & 0,139 \\
\hline Graus de Liberdade Efetivos & & & & & & $\overline{11,244}$ \\
\hline Fator de Abrangência & & & & & & 2,201 \\
\hline Incerteza Expandida & & & & & & 0,307 \\
\hline
\end{tabular}

Tabela B.32: Cálculo de incertezas para o laboratório 7 a $175^{\circ} \mathrm{C}$

\begin{tabular}{|c|c|c|c|c|c|c|}
\hline \multicolumn{7}{|c|}{ Cálculo de Incerteza: $300^{\circ} \mathrm{C}$} \\
\hline Fonte de Incerteza & Estimativa & Tipo & Distribuição & Divisor & Incerteza & G.L. \\
\hline Repetitividade & 0,231 & A & Normal & 1 & 0,230940108 & 2 \\
\hline Resolução do Equipamento do lab. & 0,1 & B & Retangular & 3,4641 & 0,028867527 & 30 \\
\hline Resolução do Artefato & 0,1 & B & Retangular & 3,4641 & 0,028867527 & 30 \\
\hline Incerteza do Indicador (herdada) & 0,2 & B & Normal & 2 & 0,1 & 30 \\
\hline Incerteza do Termopar (herdada) & 0,0 & B & Normal & 2 & 0,0 & 30 \\
\hline Incerteza Combinada & & & & & & 0,255 \\
\hline Graus de Liberdade Efetivos & & & & & & 2,964 \\
\hline Fator de Abrangência & & & & & & 4,303 \\
\hline Incerteza Expandida & & & & & & 1,097 \\
\hline
\end{tabular}

Tabela B.33: Cálculo de incertezas para o laboratório 7 a $300^{\circ} \mathrm{C}$ 


\begin{tabular}{|c|c|c|c|c|c|c|}
\hline \multicolumn{7}{|c|}{ Cálculo de Incerteza: $425^{\circ} \mathrm{C}$} \\
\hline Fonte de Incerteza & Estimativa & Tipo & Distribuição & Divisor & Incerteza & G.L. \\
\hline Repetitividade & 0,153 & $\mathrm{~A}$ & Normal & 1 & 0,152752523 & 2 \\
\hline Resolução do Equipamento do lab. & 0,1 & B & Retangular & 3,4641 & 0,028867527 & 30 \\
\hline Resolução do Artefato & 0,1 & B & Retangular & 3,4641 & 0,028867527 & 30 \\
\hline Incerteza do Indicador (herdada) & 0,2 & B & Normal & 2 & 0,1 & 30 \\
\hline Incerteza do Termopar (herdada) & 0,0 & $\mathrm{~B}$ & Normal & 2 & 0,0 & 30 \\
\hline Incerteza Combinada & & & & & & 0,187 \\
\hline Graus de Liberdade Efetivos & & & & & & 4,445 \\
\hline Fator de Abrangência & & & & & & 2,776 \\
\hline Incerteza Expandida & & & & & & 0,519 \\
\hline
\end{tabular}

Tabela B.34: Cálculo de incertezas para o laboratório 7 a $425^{\circ} \mathrm{C}$

\section{Laboratório 8}

\begin{tabular}{|c|c|c|c|c|c|c|}
\hline \multicolumn{7}{|c|}{ Cálculo de Incerteza: $25^{\circ} \mathrm{C}$} \\
\hline Fonte de Incerteza & Estimativa & Tipo & Distribuição & Divisor & Incerteza & G.L. \\
\hline Repetitividade & 0,0 & A & Normal & 1 & 0,0 & 2 \\
\hline Resolução do Equipamento do lab. & 0,01 & B & Retangular & 3,4641 & 0,028867527 & 30 \\
\hline Resolução do Artefato & 0,1 & $\mathrm{~B}$ & Retangular & 3,4641 & 0,028867527 & 30 \\
\hline Incerteza do Indicador (herdada) & 0,03 & $\mathrm{~B}$ & Normal & 2 & 0,015 & 30 \\
\hline Incerteza do Termopar (herdada) & 0,01 & $\mathrm{~B}$ & Normal & 2 & 0,005 & 30 \\
\hline Incerteza Combinada & & & & & & 0,033 \\
\hline Graus de Liberdade Efetivos & & & & & & 47,940 \\
\hline Fator de Abrangência & & & & & & 2,012 \\
\hline Incerteza Expandida & & & & & & 0,066 \\
\hline
\end{tabular}

Tabela B.35: Cálculo de incertezas para o laboratório 8 a $25^{\circ} \mathrm{C}$

\begin{tabular}{|c|c|c|c|c|c|c|}
\hline \multicolumn{7}{|c|}{ Cálculo de Incerteza: $50^{\circ} \mathrm{C}$} \\
\hline Fonte de Incerteza & Estimativa & Tipo & Distribuição & Divisor & Incerteza & G.L. \\
\hline Repetitividade & 0,010 & A & Normal & 1 & 0,010 & 2 \\
\hline Resolução do Equipamento do lab. & 0,01 & B & Retangular & 3,4641 & 0,028867527 & 30 \\
\hline Resolução do Artefato & 0,1 & B & Retangular & 3,4641 & 0,028867527 & 30 \\
\hline Incerteza do Indicador (herdada) & 0,03 & B & Normal & 2 & 0,015 & 30 \\
\hline Incerteza do Termopar (herdada) & 0,01 & B & Normal & 2 & 0,005 & 30 \\
\hline Incerteza Combinada & & & & & & 0,035 \\
\hline Graus de Liberdade Efetivos & & & & & & 47,559 \\
\hline Fator de Abrangência & & & & & & 2,012 \\
\hline Incerteza Expandida & & & & & & 0,069 \\
\hline
\end{tabular}

Tabela B.36: Cálculo de incertezas para o laboratório 8 a $50^{\circ} \mathrm{C}$

\begin{tabular}{|c|c|c|c|c|c|c|}
\hline \multicolumn{7}{|c|}{ Cálculo de Incerteza: $175^{\circ} \mathrm{C}$} \\
\hline Fonte de Incerteza & Estimativa & Tipo & Distribuição & Divisor & Incerteza & G.L. \\
\hline Repetitividade & 0,007 & A & Normal & 1 & 0,006666667 & 2 \\
\hline Resolução do Equipamento do lab. & 0,01 & $\mathrm{~B}$ & Retangular & 3,4641 & 0,028867527 & 30 \\
\hline Resolução do Artefato & 0,1 & B & Retangular & 3,4641 & 0,028867527 & 30 \\
\hline Incerteza do Indicador (herdada) & 0,03 & $\mathrm{~B}$ & Normal & 2 & 0,015 & 30 \\
\hline Incerteza do Termopar (herdada) & 0,3 & $\mathrm{~B}$ & Normal & 2 & 0,15 & 30 \\
\hline Incerteza Combinada & & & & & & 0,154 \\
\hline Graus de Liberdade Efetivos & & & & & & 32,968 \\
\hline Fator de Abrangência & & & & & & 2,037 \\
\hline Incerteza Expandida & & & & & & 0,313 \\
\hline
\end{tabular}

Tabela B.37: Cálculo de incertezas para o laboratório 8 a $175^{\circ} \mathrm{C}$ 


\begin{tabular}{|c|c|c|c|c|c|c|}
\hline \multicolumn{7}{|c|}{ Cálculo de Incerteza: $300^{\circ} \mathrm{C}$} \\
\hline Fonte de Incerteza & Estimativa & Tipo & Distribuição & Divisor & Incerteza & G.L. \\
\hline Repetitividade & 0,033 & A & Normal & 1 & 0,033333333 & 2 \\
\hline Resolução do Equipamento do lab. & 0,01 & B & Retangular & 3,4641 & 0,028867527 & 30 \\
\hline Resolução do Artefato & 0,1 & B & Retangular & 3,4641 & 0,028867527 & 30 \\
\hline Incerteza do Indicador (herdada) & 0,03 & B & Normal & 2 & 0,015 & 30 \\
\hline Incerteza do Termopar (herdada) & 0,6 & B & Normal & 2 & 0,3 & 30 \\
\hline Incerteza Combinada & & & & & & 0,304 \\
\hline Graus de Liberdade Efetivos & & & & & & 31,395 \\
\hline Fator de Abrangência & & & & & & 2,040 \\
\hline Incerteza Expandida & & & & & & 0,619 \\
\hline
\end{tabular}

Tabela B.38: Cálculo de incertezas para o laboratório 8 a $300^{\circ} \mathrm{C}$

\begin{tabular}{|c|c|c|c|c|c|c|}
\hline \multicolumn{7}{|c|}{ Cálculo de Incerteza: $425^{\circ} \mathrm{C}$} \\
\hline Fonte de Incerteza & Estimativa & Tipo & Distribuição & Divisor & Incerteza & G.L. \\
\hline Repetitividade & 0,032 & A & Normal & 1 & 0,031797973 & 2 \\
\hline Resolução do Equipamento do lab. & 0,01 & B & Retangular & 3,4641 & 0,028867527 & 30 \\
\hline Resolução do Artefato & 0,1 & B & Retangular & 3,4641 & 0,028867527 & 30 \\
\hline Incerteza do Indicador (herdada) & 0,03 & $\mathrm{~B}$ & Normal & 2 & 0,015 & 30 \\
\hline Incerteza do Termopar (herdada) & 0,6 & B & Normal & 2 & 0,3 & 30 \\
\hline Incerteza Combinada & & & & & & 0,303 \\
\hline Graus de Liberdade Efetivos & & & & & & 31,339 \\
\hline Fator de Abrangência & & & & & & 2,040 \\
\hline Incerteza Expandida & & & & & & 0,619 \\
\hline
\end{tabular}

Tabela B.39: Cálculo de incertezas para o laboratório 8 a $425^{\circ} \mathrm{C}$

\section{Laboratório 9}

\begin{tabular}{|c|c|c|c|c|c|c|}
\hline \multicolumn{7}{|c|}{ Cálculo de Incerteza: $25^{\circ} \mathrm{C}$} \\
\hline Fonte de Incerteza & Estimativa & Tipo & Distribuição & Divisor & Incerteza & G.L. \\
\hline Repetitividade & 0,1 & A & Normal & 1 & 0,1 & 2 \\
\hline Resolução do Equipamento do lab. & 0,1 & B & Retangular & 3,4641 & 0,028867527 & 30 \\
\hline Resolução do Artefato & 0,1 & B & Retangular & 3,4641 & 0,028867527 & 30 \\
\hline Incerteza do Indicador (herdada) & 0,1 & B & Normal & 2 & 0,05 & 30 \\
\hline Incerteza do Termopar (herdada) & 0,02 & B & Normal & 2 & 0,01 & 30 \\
\hline Incerteza Combinada & & & & & & 0,119 \\
\hline Graus de Liberdade Efetivos & & & & & & 4,050 \\
\hline Fator de Abrangência & & & & & & 2,776 \\
\hline Incerteza Expandida & & & & & & 0,332 \\
\hline
\end{tabular}

Tabela B.40: Cálculo de incertezas para o laboratório 9 a $25^{\circ} \mathrm{C}$

\begin{tabular}{|c|c|c|c|c|c|c|}
\hline \multicolumn{7}{|c|}{ Cálculo de Incerteza: $50^{\circ} \mathrm{C}$} \\
\hline Fonte de Incerteza & Estimativa & Tipo & Distribuição & Divisor & Incerteza & G.L. \\
\hline Repetitividade & 0,058 & A & Normal & 1 & 0,057735027 & 2 \\
\hline Resolução do Equipamento do lab. & 0,1 & $\mathrm{~B}$ & Retangular & 3,4641 & 0,028867527 & 30 \\
\hline Resolução do Artefato & 0,1 & B & Retangular & 3,4641 & 0,028867527 & 30 \\
\hline Incerteza do Indicador (herdada) & 0,1 & B & Normal & 2 & 0,05 & 30 \\
\hline Incerteza do Termopar (herdada) & 0,03 & B & Normal & 2 & 0,015 & 30 \\
\hline Incerteza Combinada & & & & & & 0,088 \\
\hline Graus de Liberdade Efetivos & & & & & & 10,268 \\
\hline Fator de Abrangência & & & & & & 2,228 \\
\hline Incerteza Expandida & & & & & & 0,196 \\
\hline
\end{tabular}

Tabela B.41: Cálculo de incertezas para o laboratório 9 a $50^{\circ} \mathrm{C}$ 


\begin{tabular}{|c|c|c|c|c|c|c|}
\hline \multicolumn{7}{|c|}{ Cálculo de Incerteza: $175^{\circ} \mathrm{C}$} \\
\hline Fonte de Incerteza & Estimativa & Tipo & Distribuição & Divisor & Incerteza & G.L. \\
\hline Repetitividade & 0,033 & A & Normal & 1 & 0,033333333 & 2 \\
\hline Resolução do Equipamento do lab. & 0,1 & B & Retangular & 3,4641 & 0,028867527 & 30 \\
\hline Resolução do Artefato & 0,1 & B & Retangular & 3,4641 & 0,028867527 & 30 \\
\hline Incerteza do Indicador (herdada) & 0,1 & $\mathrm{~B}$ & Normal & 2 & 0,05 & 30 \\
\hline Incerteza do Termopar (herdada) & 0,04 & $\mathrm{~B}$ & Normal & 2 & 0,02 & 30 \\
\hline Incerteza Combinada & & & & & & 0,075 \\
\hline Graus de Liberdade Efetivos & & & & & & 36,748 \\
\hline Fator de Abrangência & & & & & & 2,028 \\
\hline Incerteza Expandida & & & & & & 0,153 \\
\hline
\end{tabular}

Tabela B.42: Cálculo de incertezas para o laboratório 9 a $175^{\circ} \mathrm{C}$

\begin{tabular}{|c|c|c|c|c|c|c|}
\hline \multicolumn{7}{|c|}{ Cálculo de Incerteza: $300^{\circ} \mathrm{C}$} \\
\hline Fonte de Incerteza & Estimativa & Tipo & Distribuição & Divisor & Incerteza & G.L. \\
\hline Repetitividade & 0,033 & $\mathrm{~A}$ & Normal & 1 & 0,033333333 & 2 \\
\hline Resolução do Equipamento do lab. & 0,1 & $\mathrm{~B}$ & Retangular & 3,4641 & 0,028867527 & 30 \\
\hline Resolução do Artefato & 0,1 & $\mathrm{~B}$ & Retangular & 3,4641 & 0,028867527 & 30 \\
\hline Incerteza do Indicador (herdada) & 0,1 & B & Normal & 2 & 0,05 & 30 \\
\hline Incerteza do Termopar (herdada) & 0,04 & B & Normal & 2 & 0,02 & 30 \\
\hline Incerteza Combinada & & & & & & 0,075 \\
\hline Graus de Liberdade Efetivos & & & & & & 36,748 \\
\hline Fator de Abrangência & & & & & & 2,028 \\
\hline Incerteza Expandida & & & & & & 0,153 \\
\hline
\end{tabular}

Tabela B.43: Cálculo de incertezas para o laboratório 9 a $300^{\circ} \mathrm{C}$

\begin{tabular}{|c|c|c|c|c|c|c|}
\hline \multicolumn{7}{|c|}{ Cálculo de Incerteza: $425^{\circ} \mathrm{C}$} \\
\hline Fonte de Incerteza & Estimativa & Tipo & Distribuição & Divisor & Incerteza & G.L. \\
\hline Repetitividade & 0,1 & A & Normal & 1 & 0,1 & 2 \\
\hline Resolução do Equipamento do lab. & 0,1 & B & Retangular & 3,4641 & 0,028867527 & 30 \\
\hline Resolução do Artefato & 0,1 & B & Retangular & 3,4641 & 0,028867527 & 30 \\
\hline Incerteza do Indicador (herdada) & 0,1 & $\mathrm{~B}$ & Normal & 2 & 0,05 & 30 \\
\hline Incerteza do Termopar (herdada) & 0,04 & $\mathrm{~B}$ & Normal & 2 & 0,02 & 30 \\
\hline Incerteza Combinada & & & & & & 0,121 \\
\hline Graus de Liberdade Efetivos & & & & & & 4,222 \\
\hline Fator de Abrangência & & & & & & 2,776 \\
\hline Incerteza Expandida & & & & & & 0,335 \\
\hline
\end{tabular}

Tabela B.44: Cálculo de incertezas para o laboratório 9 a $425^{\circ} \mathrm{C}$

\section{Laboratório 10}

\begin{tabular}{|c|c|c|c|c|c|c|}
\hline \multicolumn{7}{|c|}{ Cálculo de Incerteza: $25^{\circ} \mathrm{C}$} \\
\hline Fonte de Incerteza & Estimativa & Tipo & Distribuição & Divisor & Incerteza & G.L. \\
\hline Repetitividade & 0,133 & A & Normal & 1 & 0,133333333 & 2 \\
\hline Resolução do Equipamento do lab. & 0,1 & B & Retangular & 3,4641 & 0,028867527 & 30 \\
\hline Resolução do Artefato & 0,1 & B & Retangular & 3,4641 & 0,028867527 & 30 \\
\hline Incerteza do Indicador (herdada) & 0,1 & B & Normal & 2 & 0,05 & 30 \\
\hline Incerteza do Termopar (herdada) & 0,04 & B & Normal & 2 & 0,02 & 30 \\
\hline Incerteza Combinada & & & & & & 0,149 \\
\hline Graus de Liberdade Efetivos & & & & & & 3,154 \\
\hline Fator de Abrangência & & & & & & 3,182 \\
\hline Incerteza Expandida & & & & & & 0,476 \\
\hline
\end{tabular}

Tabela B.45: Cálculo de incertezas para o laboratório 10 a $25^{\circ} \mathrm{C}$ 


\begin{tabular}{|c|c|c|c|c|c|c|}
\hline \multicolumn{7}{|c|}{ Cálculo de Incerteza: $50^{\circ} \mathrm{C}$} \\
\hline Fonte de Incerteza & Estimativa & Tipo & Distribuição & Divisor & Incerteza & G.L. \\
\hline Repetitividade & 0,570 & A & Normal & 1 & 0,56960025 & 2 \\
\hline Resolução do Equipamento do lab. & 0,1 & B & Retangular & 3,4641 & 0,028867527 & 30 \\
\hline Resolução do Artefato & 0,1 & B & Retangular & 3,4641 & 0,028867527 & 30 \\
\hline Incerteza do Indicador (herdada) & 0,1 & $\mathrm{~B}$ & Normal & 2 & 0,05 & 30 \\
\hline Incerteza do Termopar (herdada) & 0,04 & B & Normal & 2 & 0,02 & 30 \\
\hline Incerteza Combinada & & & & & & 0,574 \\
\hline Graus de Liberdade Efetivos & & & & & & 2,057 \\
\hline Fator de Abrangência & & & & & & 4,303 \\
\hline Incerteza Expandida & & & & & & 2,468 \\
\hline
\end{tabular}

Tabela B.46: Cálculo de incertezas para o laboratório 10 a $50^{\circ} \mathrm{C}$

\begin{tabular}{|c|c|c|c|c|c|c|}
\hline \multicolumn{7}{|c|}{ Cálculo de Incerteza: $175^{\circ} \mathrm{C}$} \\
\hline Fonte de Incerteza & Estimativa & Tipo & Distribuição & Divisor & Incerteza & G.L. \\
\hline Repetitividade & 0,067 & A & Normal & 1 & 0,066666667 & 2 \\
\hline Resolução do Equipamento do lab. & 0,1 & B & Retangular & 3,4641 & 0,028867527 & 30 \\
\hline Resolução do Artefato & 0,1 & B & Retangular & 3,4641 & 0,028867527 & 30 \\
\hline Incerteza do Indicador (herdada) & $\overline{0,1}$ & B & Normal & 2 & 0,05 & 30 \\
\hline Incerteza do Termopar (herdada) & 0,04 & B & Normal & 2 & 0,02 & 30 \\
\hline Incerteza Combinada & & & & & & 0,095 \\
\hline Graus de Liberdade Efetivos & & & & & & 8,011 \\
\hline Fator de Abrangência & & & & & & 2,306 \\
\hline Incerteza Expandida & & & & & & 0,219 \\
\hline
\end{tabular}

Tabela B.47: Cálculo de incertezas para o laboratório 10 a $175^{\circ} \mathrm{C}$

\begin{tabular}{|c|c|c|c|c|c|c|}
\hline \multicolumn{7}{|c|}{ Cálculo de Incerteza: $300^{\circ} \mathrm{C}$} \\
\hline Fonte de Incerteza & Estimativa & Tipo & Distribuição & Divisor & Incerteza & G.L. \\
\hline Repetitividade & 0,567 & A & Normal & 1 & 0,566666667 & 2 \\
\hline Resolução do Equipamento do lab. & 0,1 & $\mathrm{~B}$ & Retangular & 3,4641 & 0,028867527 & 30 \\
\hline Resolução do Artefato & 0,1 & $\mathrm{~B}$ & Retangular & 3,4641 & 0,028867527 & 30 \\
\hline Incerteza do Indicador (herdada) & 0,3 & B & Normal & 2 & 0,15 & 30 \\
\hline Incerteza do Termopar (herdada) & 1,5 & B & Normal & 2 & 0,75 & 30 \\
\hline Incerteza Combinada & & & & & & 0,953 \\
\hline Graus de Liberdade Efetivos & & & & & & 13,266 \\
\hline Fator de Abrangência & & & & & & 2,160 \\
\hline Incerteza Expandida & & & & & & 2,058 \\
\hline
\end{tabular}

Tabela B.48: Cálculo de incertezas para o laboratório 10 a $300^{\circ} \mathrm{C}$

\begin{tabular}{|c|c|c|c|c|c|c|}
\hline \multicolumn{7}{|c|}{ Cálculo de Incerteza: $425^{\circ} \mathrm{C}$} \\
\hline Fonte de Incerteza & Estimativa & Tipo & Distribuição & Divisor & Incerteza & G.L. \\
\hline Repetitividade & 0,153 & A & Normal & 1 & 0,152752523 & 2 \\
\hline Resolução do Equipamento do lab. & 0,1 & B & Retangular & 3,4641 & 0,028867527 & 30 \\
\hline Resolução do Artefato & 0,1 & B & Retangular & 3,4641 & 0,028867527 & 30 \\
\hline Incerteza do Indicador (herdada) & 0,3 & $\bar{B}$ & Normal & 2 & 0,15 & 30 \\
\hline Incerteza do Termopar (herdada) & 1,5 & $\mathrm{~B}$ & Normal & 2 & 0,75 & 30 \\
\hline Incerteza Combinada & & & & & & 0,781 \\
\hline Graus de Liberdade Efetivos & & & & & & 34,339 \\
\hline Fator de Abrangência & & & & & & 2,032 \\
\hline Incerteza Expandida & & & & & & 1,587 \\
\hline
\end{tabular}

Tabela B.49: Cálculo de incertezas para o laboratório 10 a $425^{\circ} \mathrm{C}$ 


\section{Laboratório 11}

\begin{tabular}{|c|c|c|c|c|c|c|}
\hline \multicolumn{7}{|c|}{ Cálculo de Incerteza: $25^{\circ} \mathrm{C}$} \\
\hline Fonte de Incerteza & Estimativa & Tipo & Distribuição & Divisor & Incerteza & G.L. \\
\hline Repetitividade & 0,050 & A & Normal & 1 & 0,050 & 2 \\
\hline Resolução do Equipamento do lab. & 0,01 & B & Retangular & 3,4641 & 0,028867527 & 30 \\
\hline Resolução do Artefato & 0,1 & B & Retangular & 3,4641 & 0,028867527 & 30 \\
\hline Incerteza do Indicador (herdada) & 0,6 & B & Normal & 2 & 0,3 & 30 \\
\hline Incerteza do Termopar (herdada) & 0,7 & B & Normal & 2 & 0,035 & 30 \\
\hline Incerteza Combinada & & & & & & 0,074 \\
\hline Graus de Liberdade Efetivos & & & & & & 9,266 \\
\hline Fator de Abrangência & & & & & & 2,262 \\
\hline Incerteza Expandida & & & & & & 0,167 \\
\hline
\end{tabular}

Tabela B.50: Cálculo de incertezas para o laboratório 11 a $25^{\circ} \mathrm{C}$

\begin{tabular}{|c|c|c|c|c|c|c|}
\hline \multicolumn{7}{|c|}{ Cálculo de Incerteza: $50^{\circ} \mathrm{C}$} \\
\hline Fonte de Incerteza & Estimativa & Tipo & Distribuição & Divisor & Incerteza & G.L. \\
\hline Repetitividade & 0,058 & A & Normal & 1 & 0,058118653 & 2 \\
\hline Resolução do Equipamento do lab. & 0,01 & $\mathrm{~B}$ & Retangular & 3,4641 & 0,028867527 & 30 \\
\hline Resolução do Artefato & 0,1 & B & Retangular & 3,4641 & 0,028867527 & 30 \\
\hline Incerteza do Indicador (herdada) & 0,06 & B & Normal & 2 & 0,03 & 30 \\
\hline Incerteza do Termopar (herdada) & 0,07 & $\mathrm{~B}$ & Normal & 2 & 0,035 & 30 \\
\hline Incerteza Combinada & & & & & & 0,080 \\
\hline Graus de Liberdade Efetivos & & & & & & 6,934 \\
\hline Fator de Abrangência & & & & & & 2,447 \\
\hline Incerteza Expandida & & & & & & 0,195 \\
\hline
\end{tabular}

Tabela B.51: Cálculo de incertezas para o laboratório 11 a $50^{\circ} \mathrm{C}$

\begin{tabular}{|c|c|c|c|c|c|c|}
\hline \multicolumn{7}{|c|}{ Cálculo de Incerteza: $175^{\circ} \mathrm{C}$} \\
\hline Fonte de Incerteza & Estimativa & Tipo & Distribuição & Divisor & Incerteza & G.L. \\
\hline Repetitividade & 0,043 & A & Normal & 1 & 0,043333333 & 2 \\
\hline Resolução do Equipamento do lab. & 0,01 & B & Retangular & 3,4641 & 0,028867527 & 30 \\
\hline Resolução do Artefato & 0,1 & B & Retangular & 3,4641 & 0,028867527 & 30 \\
\hline Incerteza do Indicador (herdada) & 0,06 & B & Normal & 2 & 0,03 & 30 \\
\hline Incerteza do Termopar (herdada) & 0,09 & B & Normal & 2 & 0,045 & 30 \\
\hline Incerteza Combinada & & & & & & 0,075 \\
\hline Graus de Liberdade Efetivos & & & & & & 16,339 \\
\hline Fator de Abrangência & & & & & & 2,120 \\
\hline Incerteza Expandida & & & & & & 0,159 \\
\hline
\end{tabular}

Tabela B.52: Cálculo de incertezas para o laboratório 11 a $175^{\circ} \mathrm{C}$

\begin{tabular}{|c|c|c|c|c|c|c|}
\hline \multicolumn{7}{|c|}{ Cálculo de Incerteza: $300^{\circ} \mathrm{C}$} \\
\hline Fonte de Incerteza & Estimativa & Tipo & Distribuição & Divisor & Incerteza & G.L. \\
\hline Repetitividade & 0,075 & A & Normal & 1 & 0,07535103 & 2 \\
\hline Resolução do Equipamento do lab. & 0,01 & B & Retangular & 3,4641 & 0,028867527 & 30 \\
\hline Resolução do Artefato & 0,1 & B & Retangular & 3,4641 & 0,028867527 & 30 \\
\hline Incerteza do Indicador (herdada) & 0,06 & $\mathrm{~B}$ & Normal & 2 & 0,03 & 30 \\
\hline Incerteza do Termopar (herdada) & 0,08 & $\mathrm{~B}$ & Normal & 2 & 0,04 & 30 \\
\hline Incerteza Combinada & & & & & & 0,095 \\
\hline Graus de Liberdade Efetivos & & & & & & 5,005 \\
\hline Fator de Abrangência & & & & & & 2,571 \\
\hline Incerteza Expandida & & & & & & 0,244 \\
\hline
\end{tabular}

Tabela B.53: Cálculo de incertezas para o laboratório 11 a $300^{\circ} \mathrm{C}$ 


\begin{tabular}{|c|c|c|c|c|c|c|}
\hline \multicolumn{7}{|c|}{ Cálculo de Incerteza: $425^{\circ} \mathrm{C}$} \\
\hline Fonte de Incerteza & Estimativa & Tipo & Distribuição & Divisor & Incerteza & G.L. \\
\hline Repetitividade & 0,194 & A & Normal & 1 & 0,193677854 & 2 \\
\hline Resolução do Equipamento do lab. & 0,01 & $\mathrm{~B}$ & Retangular & 3,4641 & 0,028867527 & 30 \\
\hline Resolução do Artefato & 0,1 & $\bar{B}$ & Retangular & 3,4641 & 0,028867527 & 30 \\
\hline Incerteza do Indicador (herdada) & 0,06 & $\bar{B}$ & Normal & 2 & 0,03 & 30 \\
\hline Incerteza do Termopar (herdada) & 0,08 & B & Normal & 2 & 0,04 & 30 \\
\hline Incerteza Combinada & & & & & & 0,202 \\
\hline Graus de Liberdade Efetivos & & & & & & 2,372 \\
\hline Fator de Abrangência & & & & & & 4,303 \\
\hline Incerteza Expandida & & & & & & 0,870 \\
\hline
\end{tabular}

Tabela B.54: Cálculo de incertezas para o laboratório 11 a $425^{\circ} \mathrm{C}$ 


\section{Apêndice C}

\section{Algoritmo EM}

\% Arquivo prog.m Rotinas de matlab para a obtenção de estimativas

$\%$ de máxima verossimilhança para os vetores de parâmetros alpha e beta \% \% \% \% \% \% \% \% \% \% \% \% \% \% \% \% \% \% \% \% \% \% \% \% \% \% \% \% \% \% \% \% \% \% \% \% \% \% \% \% \% \% \% \% \% \% \% \% \% \% \% \% \% \% \% \% \% \% \% \% \% \% \% \% \% \% \%

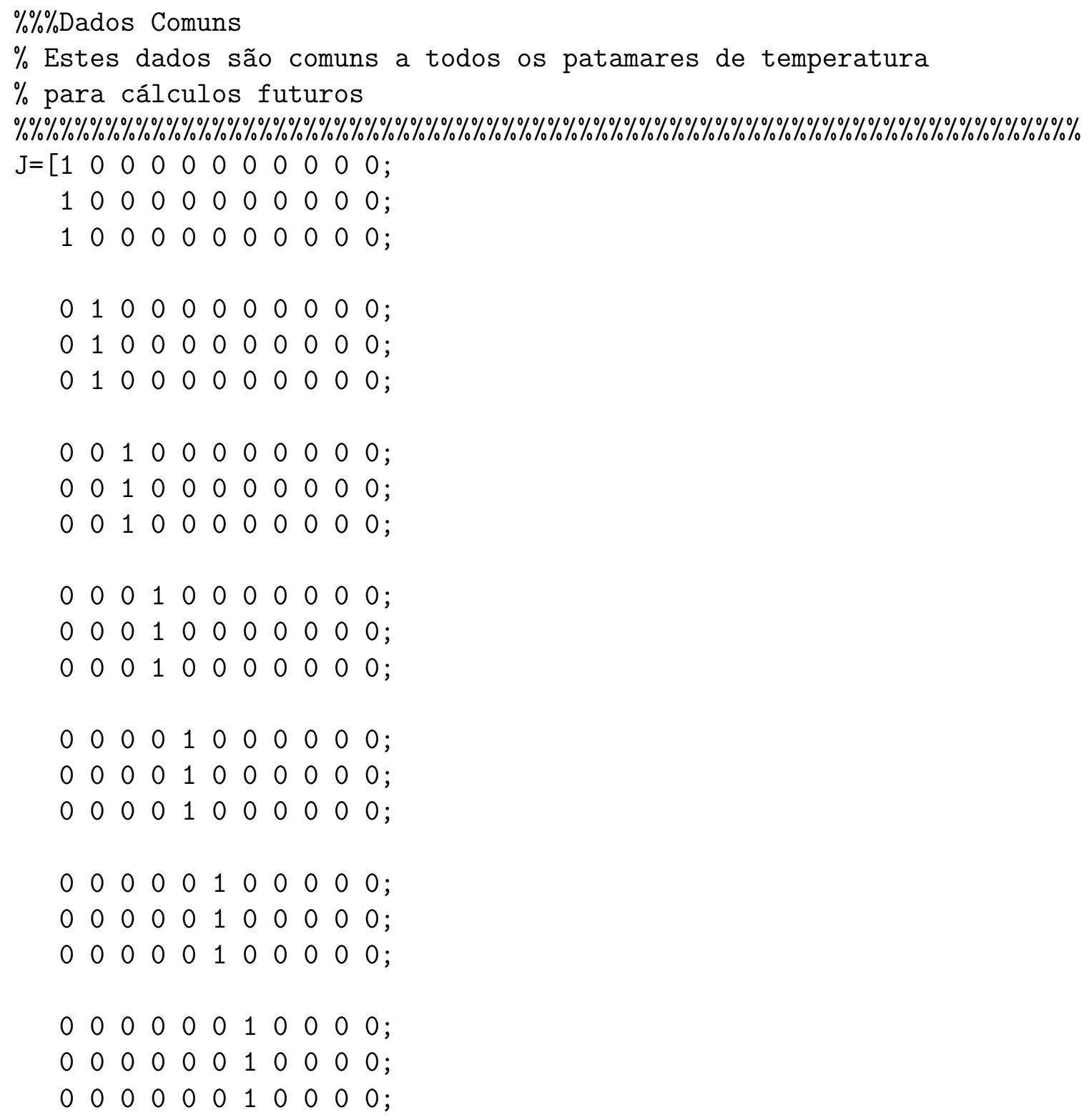




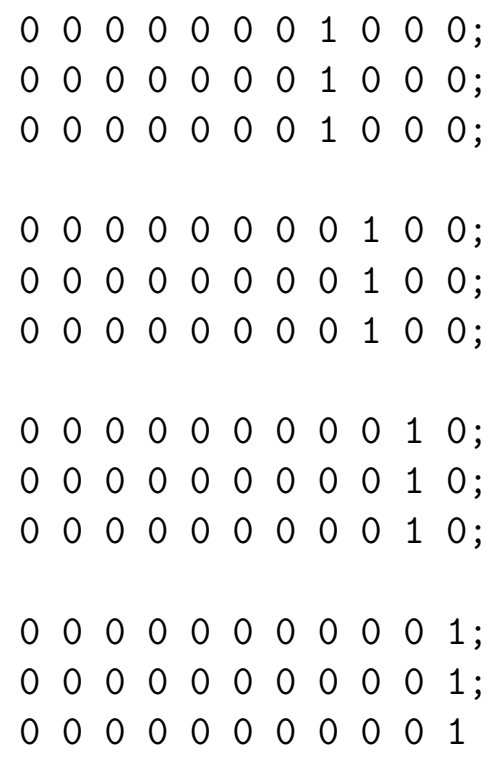

$\% \%$ Dados Para 25 Graus

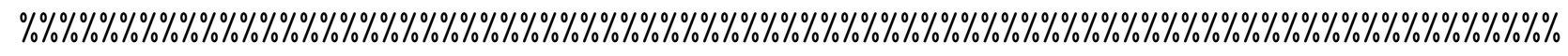
mij_25=25;

sigmaxj_25=0.1;

yj_25=[.1;.2;.2;0;.1;.1; .2;-.3;0;.5;.5;.5;0;-.2;-.2;

$-1.8 ;-1.9 ;-1.9 ;-1.8 ;-1.9 ;-1.9 ;-.05 ;-.05 ;-.05 ; .4 ; .1 ; .1 ;$

$-.7 ;-.7 ;-.7 ; .05 ;-.1 ;-.1] ;$

dj_25=[ $\begin{array}{lllllllll}0.173056 & 0.173056 & 0.173056 & 0.502681 & .502681 & .502681 & .047961\end{array}$

$\begin{array}{lllllllll}.047961 & .047961 & .014161 & .014161 & .014161 & .011025 & .011025 & .011025\end{array}$

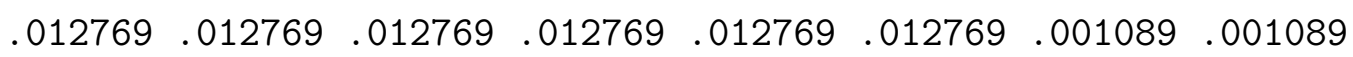

$\begin{array}{llllllllll}.001089 & .014161 & .014161 & .014161 & .022201 & .022201 & .022201 & .005476\end{array}$

$.005476 .005476]$;

for $I=1: 33$,

for $\mathrm{K}=1: 33$,

if $\mathrm{I}==\mathrm{K}$

else mdj_25(I, I $)=d j \_25(I)$; mdj_25 $(I, K)=0$; end

end

end

DJ_25=inv(mdj_25); $\%$ inversa da matriz mdj_25

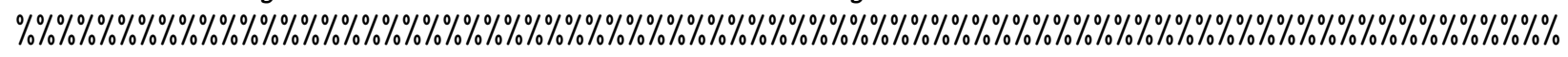




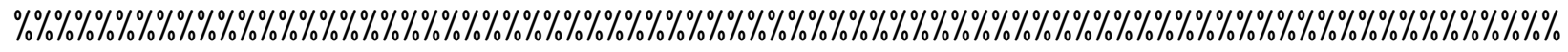
mij_50=50;

sigmaxj_50=0.2015565;

yj_50 $=[.65 ; .15 ; .05 ; .15 ; .25 ;-.65 ; .25 ;-.05 ; .05 ; .15 ; .15$;

$.05 ; .05 ;-.25 ;-.15 ;-1.55 ;-1.35 ;-1.45 ;-1.55 ;-1.35 ;-1.45$;

$-.12 ;-.12 ;-.09 ; .25 ; .05 ; .15 ;-.65 ;-.75 ;-.85 ;-.05 ; .07$;

$.15]$;

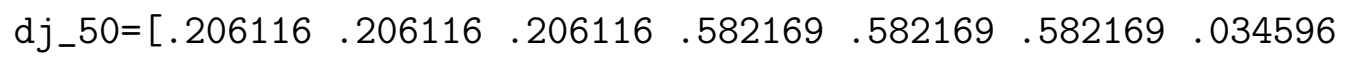

$\begin{array}{llllllllll}.034596 & .034596 & .015376 & .015376 & .015376 & .0144 & .0144 & .0144 & .012544\end{array}$

$\begin{array}{lllllllll}.012544 & .012544 & .014884 & .014884 & .014884 & .001225 & .001225 & .001225\end{array}$

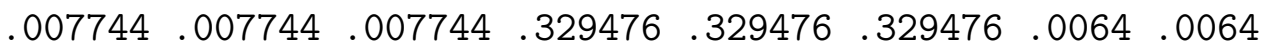

$.0064]$

for $I=1: 33$,

for $K=1: 33$,

if $\mathrm{I}==\mathrm{K}$ mdj_50 (I, I) $=$ dj_50(I) ;

else mdj_50 (I, K) $=0$;

end

end

end

DJ_50=inv(mdj_50); $\%$ inversa da matriz mdj_50

\% \% \% \% \% \% \% \% \% \% \% \% \% \% \% \% \% \% \% \% \% \% \% \% \% \% \% \% \% \% \% \% \% \% \% \% \% \% \% \% \% \% \% \% \% \% \% \% \% \% \% \% \% \% \% \% \% \% \% \% \% \% \% \% \% \% \% \% \% \% \% \% \% \% \%

$\% \%$ Dados Para 175 Graus

\%\% \% \% \% \% \% \% \% \% \% \% \% \% \% \% \% \% \% \% \% \% \% \% \% \% \% \% \% \% \% \% \% \% \% \% \% \% \% \% \% \% \% \% \% \% \% \% \% \% \% \% \% \% \% \% \% \% \% \% \% \% \% \% \% \% \% \% \% \% \% \% mij_175=175;

sigmaxj_175=0.2015565;

yj_175 $=[-2.35 ;-2.55 ;-2.95 ;-1.65 ;-1.25 ;-1.85 ;-2.15 ;-.85$;

$-2.15 ;-2.65 ;-2.65 ;-2.85 ;-2.55 ;-2.55 ;-2.35 ;-1.45 ;-1.35$;

$-1.65 ;-1.45 ;-1.35 ;-1.65 ;-.79 ;-.79 ;-.77 ; .25 ; .35 ; .25$;

$-1.05 ;-1.05 ;-.85 ;-.1 ;-.02 ; .05]$;

dj_175=[ $\begin{array}{llllllll}.2025 & .2025 & .2025 & .5329 & .5329 & .5329 & .214369 & .214369\end{array}$

$\begin{array}{lllllllll}.214369 & .019044 & .019044 & .019044 & .018496 & .018496 & .018496 & .019321\end{array}$

$\begin{array}{lllllllll}.019321 & .019321 & .019321 & .019321 & .019321 & .023716 & .023716 & .023716\end{array}$

$\begin{array}{lllllllll}.005625 & .005625 & .005625 & .009025 & .009025 & .009025 & .005625 & .005625\end{array}$

$.005625]$

for $I=1: 33$,

for $K=1: 33$,

if $\mathrm{I}==\mathrm{K}$ mdj_175(I, I) $=d_{-} \_175(I)$;

else

mdj_175 $(I, K)=0$; 
end

end

end

DJ_175=inv(mdj_175); \%inversa da matriz mdj_175

\% \% \% \% \% \% \% \% \% \% \% \% \% \% \% \% \% \% \% \% \% \% \% \% \% \% \% \% \% \% \% \% \% \% \% \% \% \% \% \% \% \% \% \% \% \% \% \% \% \% \% \% \% \% \% \% \% \% \% \% \% \% \% \% \% \% \% \% \% \% \% \% \%

$\% \% \%$ Dados Para 300 Graus

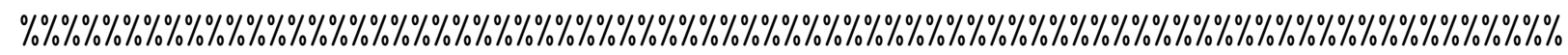
mij_300=300;

sigmaxj_300=.3640055;

yj_300 $=[-2.6 ;-3.4 ;-3.6 ;-2.2 ;-1.5 ;-1.9 ; .5 ;-.3 ;-.2 ;-1.6$;

$-1.7 ;-2 ;-1.9 ;-2.2 ;-2.2 ;-1.6 ;-1.2 ;-2 ;-1.6 ;-1.2 ;-2 ;-1.21$;

$-1.21 ;-1.11 ; 1 ; .9 ; .9 ;-.3 ;-2 ;-2 ;-.1 ;-.21 ; .05]$;

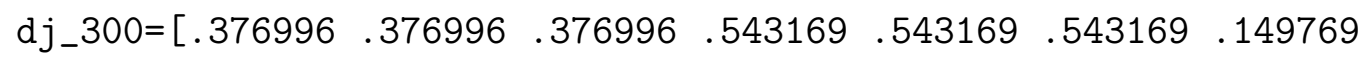

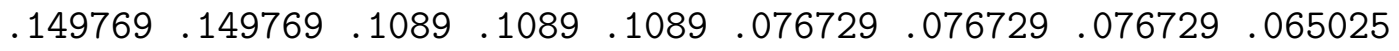

$\begin{array}{lllllllll}.065025 & .065025 & .065025 & .065025 & .065025 & .092416 & .092416 & .092416\end{array}$

$\begin{array}{llllllllll}.005625 .005625 & .005625 & .008209 & .008209 & .008209 & .009025 & .00925\end{array}$

$.009025]$

for $I=1: 33$,

for $K=1: 33$,

if $\mathrm{I}==\mathrm{K}$

mdj_300 (I, I) $=$ dj_300(I);

else

mdj_300 $(I, K)=0$;

end

end

end

DJ_300=inv(mdj_300); $\%$ inversa da matriz mdj_300

\% \% \% \% \% \% \% \% \% \% \% \% \% \% \% \% \% \% \% \% \% \% \% \% \% \% \% \% \% \% \% \% \% \% \% \% \% \% \% \% \% \% \% \% \% \% \% \% \% \% \% \% \% \% \% \% \% \% \% \% \% \% \% \% \% \% \% \% \%

$\% \%$ Dados Para 425 Graus

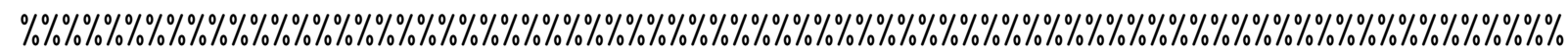
mij_425= 425;

sigmaxj_425=.3640055;

yj_425 $=[-3.85 ;-3.75 ;-3.75 ;-2.45 ;-1.85 ;-1.95 ; 1.55 ; 1.55 ;$

$1.55 ;-.95 ;-.85 ;-1.05 ;-1.55 ;-1.95 ;-1.25 ;-1.75 ;-1.85 ;-2.25$;

$-1.75 ;-1.85 ;-2.25 ;-1.87 ;-1.86 ;-1.96 ; 1.65 ; 1.65 ; 1.95 ;-.55 ;$

$-.55 ;-.65 ;-.55 ;-.59 ; .01] ;$

dj_425=[ $\begin{array}{llllllll}.285156 & .285156 & .285156 & 1.485961 & 1.485961 & 1.485961 & .094249\end{array}$

$\begin{array}{lllllllllll}.094249 .094249 & .097344 & .097344 & .097344 & .107584 & .07584 & .107584\end{array}$

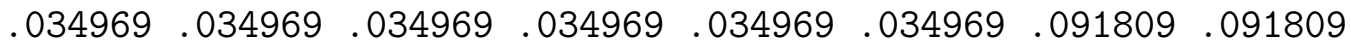




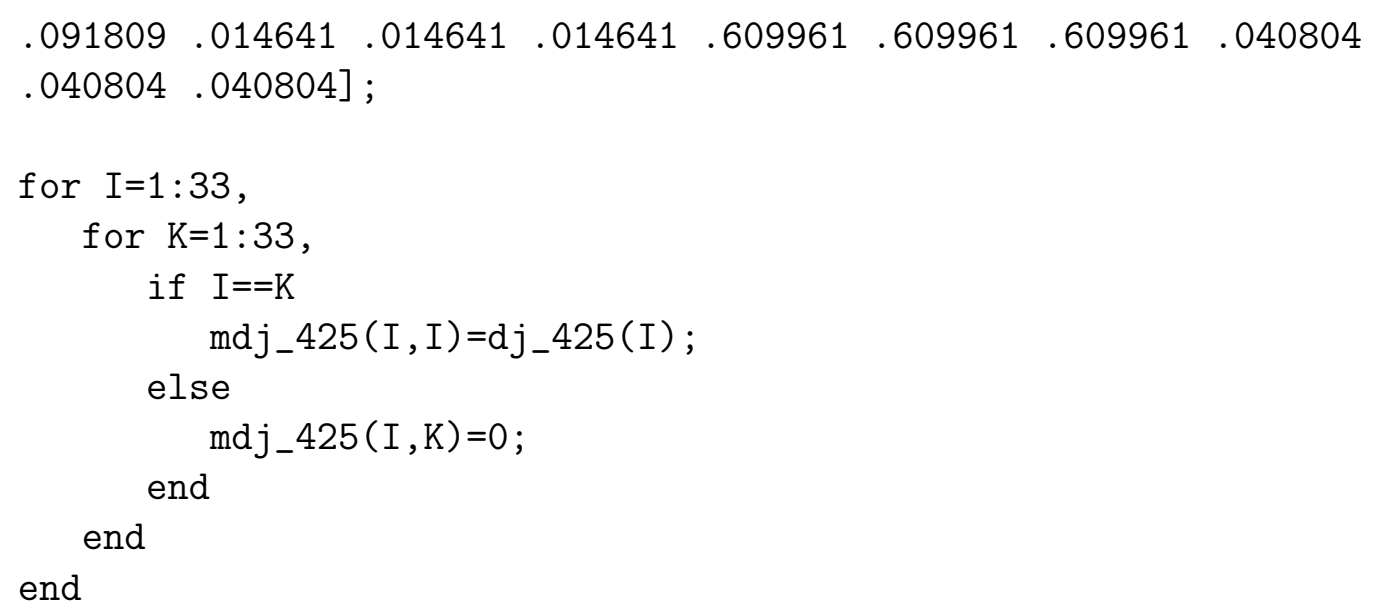

DJ_425=inv(mdj_425); \%inversa da matriz mdj_425

\% \% \% \% \% \% \% \% \% \% \% \% \% \% \% \% \% \% \% \% \% \% \% \% \% \% \% \% \% \% \% \% \% \% \% \% \% \% \% \% \% \% \% \% \% \% \% \% \% \% \% \% \% \% \% \% \% \% \% \% \% \% \% \% \% \% \% \% \% \% \% \% \%

\% \% \% \% \% \% \% \% \% \% \% \% \% \% \% \% \% \% \% \% \% \% \% \% \% \% \% \% \% \% \% \% \% \% \% \% \% \% \% \% \% \% \% \% \% \% \% \% \% \% \% \% \% \% \% \% \% \% \% \% \% \% \% \% \% \% \% \% \% \% \% $\% \%$ Chutes iniciais

$\%$ Aqui atribuímos valores iniciais para o vetor alpha e para o vetor beta $\%$ para a primeira iteração

\% \% \% \% \% \% \% \% \% \% \% \% \% \% \% \% \% \% \% \% \% \% \% \% \% \% \% \% \% \% \% \% \% \% \% \% \% \% \% \% \% \% \% \% \% \% \% \% \% \% \% \% \% \% \% \% \% \% \% \% \% \% \% \% \% \% \% \% \% \% \% \% \% \%

alpha1 $=0 ;$ alpha2 $=0 ;$ alpha3 $=0 ;$ alpha4 $=0 ;$ alpha5 $=0 ;$

alpha6 $=0 ;$ alpha $7=0 ;$ alpha $8=0 ;$ alpha9 $=0 ;$ alpha10 $=0 ;$

alpha11 = 0;

beta $1=0 ;$ beta $2=0 ;$ beta $3=0 ;$ beta $4=0 ;$ beta $5=0 ;$ beta $6=0$;

beta $7=0 ; \operatorname{beta} 8=0 ; \operatorname{beta} 9=0 ; \operatorname{beta10}=0 ;$ beta11 $=0$;

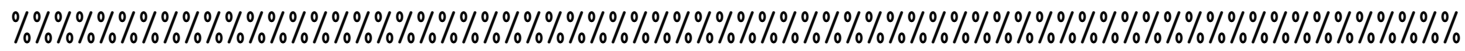

stop=0; \% Flag que será usado na obtenção do erro desejado

\% Sinalizará o fim do loop

loop $=0 ; \%$ Contador do número de loop realizado

ERRO_MAX=1.e-30; \% Erro máximo desejado

\% \% \% \% \% \% \% \% \% \% \% \% \% \% \% \% \% \% \% \% \% \% \% \% \% \% \% \% \% \% \% \% \% \% \% \% \% \% \% \% \% \% \% \% \% \% \% \% \% \% \% \% \% \% \% \% \% \% \% \% \% \% \% \% \% \% \% \% \% \% \% \% \% \% \%

\%Aqui começam as iterações

while stop $\sim 1$,

loop=loop+1;

\% \% \% \% \% \% \% \% \% \% \% \% \% \% \% \% \% \% \% \% \% \% \% \% \% \% \% \% \% \% \% \% \% \% \% \% \% \% \% \% \% \% \% \% \% \% \% \% \% \% \% \% \% \% \% \% \% \% \% \% \% \% \% \% \% \% \%

$\%$ Os vetores miyj serão gerados pela função genmiyj

$\%$ utilizando os valores de alpha1, alpha2, alpha3, alpha4, alpha5, 
\% alpha6, alpha7, alpha8, alpha9, alpha10, alpha11, beta1, beta2, $\%$ beta3, beta4, beta5, beta6, beta7, beta8, beta9, beta10, beta11 $\%$ e mij para cada patamar de temperatura.

miyj_25=genmiyj (alpha1, alpha2, alpha3, alpha4, alpha5, alpha6, alpha7, alpha8, alpha9, alpha10, alpha11, beta1, beta2 , beta3, beta4, beta5, beta6, beta7, beta8, beta9, beta10, beta11,mij_25);

miyj_50=genmiyj (alpha1, alpha2, alpha3, alpha4, alpha5, alpha6, alpha7 , alpha8, alpha9, alpha10, alpha11, beta1, beta2, beta3, beta4, beta5, beta6, beta7, beta8, beta9, beta10, beta11,mij_50);

miyj_175=genmiyj (alpha1, alpha2, alpha3, alpha4, alpha5, alpha6, alpha7, alpha8, alpha9, alpha10, alpha11, beta1, beta2, beta3, beta4, beta5, beta6 , beta7, beta8, beta9, beta10, beta11,mij_175);

miyj_300=genmiyj (alpha1, alpha2, alpha3, alpha4, alpha5, alpha6, alpha7, alpha8, alpha9, alpha10, alpha11, beta1, beta2, beta3, beta4, beta5, beta6, beta7, beta8, beta9, beta10, beta11,mij_300);

miyj_425=genmiyj (alpha1, alpha2, alpha3, alpha4, alpha5, alpha6, alpha7, alpha8, alpha9, alpha10, alpha11, beta1, beta2, beta3, beta4, beta5, beta6 , beta7, beta8, beta9, beta10, beta11,mij_425);

\% \% \% \% \% \% \% \% \% \% \% \% \% \% \% \% \% \% \% \% \% \% \% \% \% \% \% \% \% \% \% \% \% \% \% \% \% \% \% \% \% \% \% \% \% \% \% \% \% \% \% \% \% \% \% \% \% \% \% \% \% \% \% \% \% \% \% \% \% \% \% \% \% \% \% \% \% \% \%

\% Construção do vetor beta

beta=[beta1; beta1; beta1; beta2; beta2; beta2; beta3; beta3; beta3; beta4; beta4; beta4; beta5; beta5; beta5; beta6; beta6; beta6; beta7; beta7; beta7; beta8; beta8; beta8; beta9; beta9; beta9; beta10; beta10; beta10; beta11; beta11; beta11];

alpha=[alpha1; alpha1; alpha1; alpha2; alpha2; alpha2; alpha3; alpha3; alpha3; alpha4; alpha4; alpha4; alpha5; alpha5; alpha5; alpha6; alpha6; alpha6; alpha7;alpha7; alpha7; alpha8; alpha8; alpha8; alpha9; alpha9; alpha9; alpha10; alpha10;alpha10; alpha11; alpha11; alpha11];

\section{\% \% \% \% \% \% \% \% \% \% \% \% \% \% \% \% \% \% \% \% \% \% \% \% \% \% \% \% \% \% \% \% \% \% \% \% \% \% \% \% \% \% \% \% \% \% \% \% \% \% \% \% \% \% \% \% \% \% \% \% \% \% \% \% \% \% \%}

\% Cálculo de $\mathrm{E}(\mathrm{X} / \mathrm{Yj}=\mathrm{yj})$ para a temperatura $25^{\circ} \mathrm{C}$

EX_25=mij_25+( (transp (beta) $*$ sigmaxj_25^2)*

(DJ_25 - (( (DJ_25*beta) $*($ transp $($ beta $) *$ DJ_25) $*$ sigmaxj_25^2)/

$\left(1+\operatorname{transp}(\right.$ beta $) * D J \_25 *$ beta*sigmaxj_25^2)) $\left.*\left(y j \_25-m i y j \_25\right)\right)$;

\% Cálculo de $\mathrm{E}(\mathrm{X} / \mathrm{Yj}=\mathrm{yj})$ para a temperatura $50^{\circ} \mathrm{C}$ 
EX_50=mij_50+( (transp (beta) $*$ sigmaxj_50^2)*

$\left(\right.$ DJ_50 - (( (DJ_50*beta) $*\left(\right.$ transp $($ beta $\left.) * D J \_50\right) *$ sigmaxj_50^2)/

$(1+\operatorname{transp}($ beta) $*$ DJ_50*beta*sigmaxj_50^2)))*(yj_50-miyj_50)) ;

\% Cálculo de $\mathrm{E}(\mathrm{X} / \mathrm{Yj}=\mathrm{yj})$ para a temperatura $175^{\circ} \mathrm{C}$

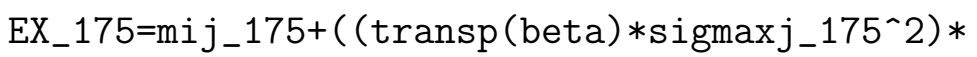

(DJ_175 - (( (DJ_175*beta) $*\left(\operatorname{transp}(\right.$ beta $\left.) * D J_{-} 175\right) * \operatorname{sigmaxj}$ _175^2)/

$\left(1+\operatorname{transp}(\right.$ beta $\left.\left.\left.\left.) * \mathrm{DJ} \_175 * \operatorname{beta} * \operatorname{sigmaxj}{ }_{1} 175^{\wedge} 2\right)\right)\right) *\left(y j \_175-m i y j \_175\right)\right)$;

\% Cálculo de $\mathrm{E}(\mathrm{X} / \mathrm{Yj}=\mathrm{yj})$ para a temperatura $300^{\circ} \mathrm{C}$

EX_300=mij_300+ ((transp $($ beta $) *$ sigmaxj_300^2) $*$

(DJ_300 - (( (DJ_300*beta) $*($ transp (beta) $*$ DJ_300)*sigmaxj_300^2)/

$\left(1+\operatorname{transp}(\right.$ beta $) * \mathrm{DJ} \_300 *$ beta*sigmaxj_300^2)) $\left.*\left(y j \_300-m i y j \_300\right)\right)$;

\% Cálculo de $E(X / Y j=y j)$ para a temperatura $425^{\circ} \mathrm{C}$

EX_425=mij_425+((transp (beta) $*$ sigmaxj_425^2)*

(DJ_425 - (( (DJ_425*beta) $*\left(\operatorname{transp}(\right.$ beta $\left.) * D J_{-} 425\right) * \operatorname{sigmaxj}$ 425^$\left.^{\wedge} 2\right) /$

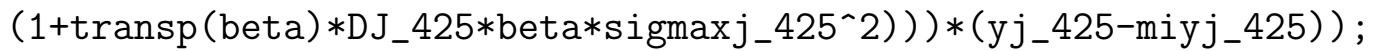

\% \% \% \% \% \% \% \% \% \% \% \% \% \% \% \% \% \% \% \% \% \% \% \% \% \% \% \% \% \% \% \% \% \% \% \% \% \% \% \% \% \% \% \% \% \% \% \% \% \% \% \% \% \% \% \% \% \% \% \% \% \% \% \% \% \% \% \% \% \% \% \% \% \% \% \%

\% Cáculo de $\operatorname{Var}(\mathrm{X} / \mathrm{Yj}=\mathrm{yj})$ para cada patamar de temperatura

$\operatorname{varx} \_25=\operatorname{sigmax} j \_25^{\wedge} 2-\left(\operatorname{transp}(\operatorname{beta}) *\left(\operatorname{sigmax} j \_25^{\wedge} 2\right)^{\wedge} 2\right) *$

(DJ_25 - ((DJ_25* (beta) $) *\left(\operatorname{transp}(\right.$ beta $\left.\left.) * D J \_25\right)\right) *($ sigmaxj_25^2)/

$(1+\operatorname{transp}($ beta $) *$ DJ_25*beta*sigmaxj_25^2) $) *$ beta;

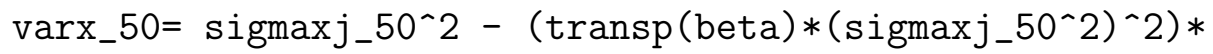

$($ DJ_50 - ( (DJ_50*(beta) $) *\left(\operatorname{transp}(\right.$ beta $\left.\left.) * D J \_50\right)\right) *($ sigmaxj_50^2)/

$(1+\operatorname{transp}($ beta $) *$ DJ_50*beta*sigmaxj_50^2))*beta;

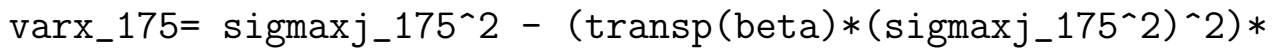

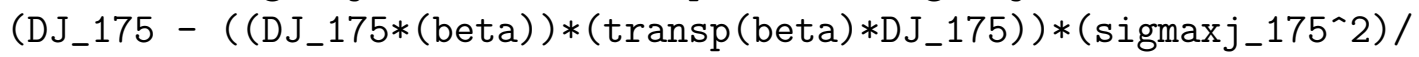

$(1+\operatorname{transp}($ beta $) *$ DJ_175*beta*sigmaxj_175^2))*beta;

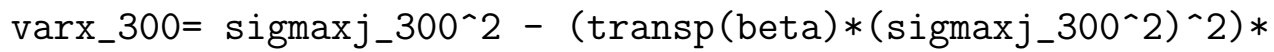

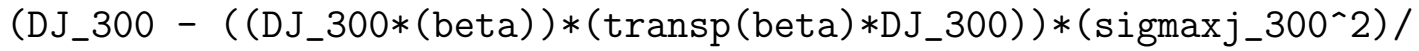

$(1+\operatorname{transp}($ beta $) *$ DJ_300*beta*sigmaxj_300^2))*beta;

$\operatorname{varx} x_{-} 425=\operatorname{sigmax} j_{-} 425^{\wedge} 2-\left(\operatorname{transp}(\operatorname{beta}) *\left(\operatorname{sigmax} j_{-} 425^{\wedge} 2\right)^{\wedge} 2\right) *$

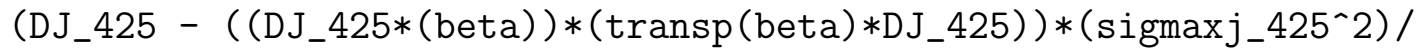

$\left(1+\operatorname{transp}(\right.$ beta $) * D_{\text {J_4 }} 425 *$ beta*sigmaxj_425^2))*beta;

\% Cálculo de $E\left(X^{\wedge} 2 / Y j=y j\right)$ para cada patamar de temperatura 
EX2_25=varx_25+EX_25^2;

EX2_50=varx_50+EX_50^2;

EX2_175=varx_175+EX_175^2;

$\mathrm{EX} 2 \_300=$ varX_300+EX_300^2;

EX2_425=varx_425+EX_425^2;

\% \% \% \% \% \% \% \% \% \% \% \% \% \% \% \% \% \% \% \% \% \% \% \% \% \% \% \% \% \% \% \% \% \% \% \% \% \% \% \% \% \% \% \% \% \% \% \% \% \% \% \%

\% Cálculo do novo valor do vetor de parâmetros beta

\%Obs.: Devido a grande extensão desta expressão, a mesma, \%por conveniência de programação, foi dividida em partes part1=inv (EX2_25*transp (J)*DJ_25*J + EX2_50*transp (J)*DJ_50*J + EX2_175*transp $(\mathrm{J}) * \mathrm{DJ} \_175 * \mathrm{~J}+\mathrm{EX} 2 \_300 * \operatorname{transp}(\mathrm{J}) * \mathrm{DJ} \_300 * \mathrm{~J}+$ EX2_425*transp $\left.(\mathrm{J}) * \mathrm{DJ} \_425 * \mathrm{~J}\right)$;

part2 $=\left(E X \_25 * \operatorname{transp}(\mathrm{J}) * \mathrm{DJ}_{-} 25 * \mathrm{~J}+\mathrm{EX} \_50 * \operatorname{transp}(\mathrm{J}) * \mathrm{DJ} \_50 * \mathrm{~J}+\right.$ EX_175*transp $(\mathrm{J}) * \mathrm{DJ} \_175 * \mathrm{~J}+\mathrm{EX} \_300 * \operatorname{transp}(\mathrm{J}) * \mathrm{DJ} \_300 * \mathrm{~J}+$ EX_425*transp $(\mathrm{J}) * \mathrm{DJ}_{-}$425*J);

$\operatorname{part3}=\operatorname{inv}\left(\operatorname{transp}(\mathrm{J}) * \mathrm{DJ} \_25 * \mathrm{~J}+\operatorname{transp}(\mathrm{J}) * \mathrm{DJ} \_50 * \mathrm{~J}+\right.$ $\left.\operatorname{transp}(\mathrm{J}) * \mathrm{DJ} \_175 * \mathrm{~J}+\operatorname{transp}(\mathrm{J}) * \mathrm{DJ} \_300 * \mathrm{~J}+\operatorname{transp}(\mathrm{J}) * \mathrm{DJ} \mathrm{J}_{-} 425 * \mathrm{~J}\right)$;

part4 $=\left(E_{-} \_25 * \operatorname{transp}(\mathrm{J}) * \mathrm{DJ} \_25 * \mathrm{~J}+\mathrm{EX} \_50 * \operatorname{transp}(\mathrm{J}) * \mathrm{DJ} \_50 * \mathrm{~J}+\right.$ EX_175*transp $(\mathrm{J}) * \mathrm{DJ} \_175 * \mathrm{~J}+\mathrm{EX} \_300 * \operatorname{transp}(\mathrm{J}) * \mathrm{DJ} \_300 * \mathrm{~J}+$ EX_425*transp (J)*DJ_425*J);

part5=inv $\left(E X 2 \_25 * \operatorname{transp}(\mathrm{J}) * \mathrm{DJ} \_25 * \mathrm{~J}+\mathrm{EX} 2 \_50 * \operatorname{transp}(\mathrm{J}) * \mathrm{DJ} \_50 * \mathrm{~J}+\right.$ EX2_175*transp $(\mathrm{J}) * \mathrm{DJ} \_175 * \mathrm{~J}+\mathrm{EX} 2 \_300 * \operatorname{transp}(\mathrm{J}) * \mathrm{DJ} \_300 * \mathrm{~J}+$ EX2_425*transp $\left.(\mathrm{J}) * \mathrm{DJ} \_425 * \mathrm{~J}\right)$;

part6 $=\left(E X \_25 * \operatorname{transp}(\mathrm{J}) * \mathrm{DJ} \_25 * \mathrm{yj}\right.$ _25 + EX_50*transp $(\mathrm{J}) * \mathrm{DJ} \_50 * \mathrm{yj}$ _50 + EX_175*transp (J)*DJ_175*yj_175 + EX_300*transp (J)*DJ_300*yj_300 + EX_425*transp (J)*DJ_425*yj_425);

$\operatorname{part7}=\operatorname{inv}\left(E X 2 \_25 * \operatorname{transp}(\mathrm{J}) * \mathrm{DJ} \_25 * \mathrm{~J}+\mathrm{EX} 2 \_50 * \operatorname{transp}(\mathrm{J}) * \mathrm{DJ} \_50 * \mathrm{~J}+\right.$ EX2_175*transp $(\mathrm{J}) * \mathrm{DJ} \_175 * \mathrm{~J}+\mathrm{EX} 2 \_300 * \operatorname{transp}(\mathrm{J}) * \mathrm{DJ} \_300 * \mathrm{~J}+$ EX2_425*transp $\left.(\mathrm{J}) * \mathrm{DJ} \_425 * \mathrm{~J}\right)$;

part8 $=\left(E X \_25 * \operatorname{transp}(\mathrm{J}) * \mathrm{DJ} \_25 * \mathrm{~J}+\mathrm{EX} \_50 * \operatorname{transp}(\mathrm{J}) * \mathrm{DJ} \_50 * \mathrm{~J}+\right.$ $\operatorname{EX} \_175 * \operatorname{transp}(\mathrm{J}) * \mathrm{DJ} \_175 * \mathrm{~J}+\mathrm{EX} \_300 * \operatorname{transp}(\mathrm{J}) * \mathrm{DJ} \_300 * \mathrm{~J}+$ EX_425*transp (J)*DJ_425*J); 
$\operatorname{part9}=\operatorname{inv}\left(\operatorname{transp}(\mathrm{J}) * \mathrm{DJ} \_25 * \mathrm{~J}+\operatorname{transp}(\mathrm{J}) * \mathrm{DJ} \_50 * \mathrm{~J}+\operatorname{transp}(\mathrm{J}) * \mathrm{DJ} \_175 * \mathrm{~J}\right.$

$\left.+\operatorname{transp}(\mathrm{J}) * \mathrm{DJ} \_300 * \mathrm{~J}+\operatorname{transp}(\mathrm{J}) * \mathrm{DJ}_{-} 425 * \mathrm{~J}\right)$;

$\operatorname{part10}=\left(\operatorname{transp}(J) * D J_{-} 25 * y j \_25+\operatorname{transp}(J) * D J \_50 * y j \_50+\right.$

$\operatorname{transp}(\mathrm{J}) * \mathrm{DJ} \mathrm{J}_{1} 175 * \mathrm{yj} \mathrm{y}_{1} 175+\operatorname{transp}(\mathrm{J}) * \mathrm{DJ} \mathrm{J}_{-} 300 * \mathrm{y} \mathrm{j}_{-} 300+$

$\operatorname{transp}(\mathrm{J}) * \mathrm{DJ}$ _ $\left._{4} 425 * \mathrm{y} j_{-} 425\right)$;

newbeta=inv $(111-\operatorname{part} 1 *$ part $2 *$ part $3 *$ part 4$) *$

(part5*part6-part7*part8*part9*part10);

\%Fim do cálculo do novo valor do vetor de parâmetros beta

$\% \% \% \% \% \% \% \% \% \% \% \% \% \% \% \% \% \% \% \% \% \% \% \% \% \% \% \% \% \% \% \% \% \% \% \% \% \% \% \% \% \% \% \% \% \% \% \% \% \% \% \% \%$

\%Cálculo do novo valor do vetor de parâmetros alpha

part11 $=\left(\right.$ EX_25*transp $(\mathrm{J}) * \mathrm{DJ} \_25 * \mathrm{~J} *$ newbeta +

EX_50*transp $(\mathrm{J}) * \mathrm{DJ}$ _50 $* \mathrm{~J} *$ newbeta + EX_175*transp $(\mathrm{J}) * \mathrm{DJ} \_175 * \mathrm{~J} *$ newbeta +

EX_300*transp $(\mathrm{J}) * \mathrm{DJ} \_300 * \mathrm{~J} *$ newbeta $+\operatorname{EX} \_425 * \operatorname{transp}(\mathrm{J}) * \mathrm{DJ} \_425 * \mathrm{~J} *$ newbeta) ;

newalpha $=$ part $3 *($ part 10-part11);

\%\%im do cálculo do novo valor do vetor de parâmetros alpha

\% \% \% \% \% \% \% \% \% \% \% \% \% \% \% \% \% \% \% \% \% \% \% \% \% \% \% \% \% \% \% \% \% \% \% \% \% \% \% \% \% \% \% \% \% \% \% \% \% \% \% \% \% \% \% \% \% \% \% \% \% \% \%

newbeta3 $=[$ newbeta $(1) ; \operatorname{newbeta}(1) ; \operatorname{newbeta}(1) ; \operatorname{newbeta}(2) ; \operatorname{newbeta}(2)$;

newbeta (2); newbeta (3); newbeta (3); newbeta (3); newbeta (4); newbeta (4);

newbeta (4); newbeta (5); newbeta (5); newbeta (5); newbeta (6);newbeta (6);

newbeta (6); newbeta (7); newbeta (7);newbeta (7); newbeta (8);newbeta (8);

newbeta (8); newbeta (9); newbeta (9); newbeta (9); newbeta (10); newbeta (10);

newbeta (10); newbeta (11); newbeta (11); newbeta(11)] ;

newalpha3= [newalpha(1); newalpha(1); newalpha(1); newalpha (2); newalpha (2); newalpha (2) ; newalpha (3); newalpha (3) ; newalpha (3) ; newalpha (4); newalpha (4); newalpha (4); newalpha (5) ; newalpha (5); newalpha (5); newalpha (6) ; newalpha (6); newalpha (6); newalpha (7); newalpha (7); newalpha (7); newalpha (8); newalpha (8); newalpha (8); newalpha (9); newalpha (9); newalpha (9); newalpha (10); newalpha (10); newalpha(10); newalpha(11); newalpha(11); newalpha(11)];

$\% \%$ Critério de Parada do Algoritmo EM

\%\% Matrizes de Covariâncias atualizadas

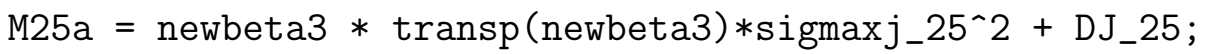

$\mathrm{M} 50 \mathrm{a}=$ newbeta3 $* \operatorname{transp}($ newbeta3 $) *$ sigmaxj_50^2 + DJ_50;

M175a $=$ newbeta3 $* \operatorname{transp}($ newbeta3 $) *$ sigmaxj_175^2 + DJ_175; 
M300a $=$ newbeta3 $* \operatorname{transp}($ newbeta3 $) * \operatorname{sigmaxj} \_300^{\wedge} 2+$ DJ_300;
M425a $=$ newbeta3 $* \operatorname{transp}($ newbeta3 $) * \operatorname{sigmaxj} 4425^{\wedge} 2+$ DJ_425;

$\% \%$ Matrizes da iteração anterior

M25 = beta*transp (beta) $*$ sigmaxj_25^2 + DJ_25;

M50 = beta*transp (beta) $*$ sigmaxj_50^2 + DJ_50;

M175 = beta*transp (beta) $*$ sigmaxj_175^2 + DJ_175;

M300 = beta*transp (beta) $*$ sigmaxj_300^2 + DJ_300;

M425 = beta*transp (beta) $*$ sigmaxj_425^2 + DJ_425;

$\% \%$ Log da verossimilhança da iteração atual

Lfatual $=(-165 / 2) * \log (2 * \mathrm{pi})-(1 / 2) *(\log (\operatorname{det}($ M25a $))+\log (\operatorname{det}($ M50a $))+$

$\log (\operatorname{det}($ M175a) $)+\log (\operatorname{det}($ M300a $))+\log (\operatorname{det}($ M425a)) $)-(1 / 2) *$

(transp(yj_25 - miyj_25)* inv(M25a)* (yj_25 - miyj_25) +

$\operatorname{transp}\left(y j_{-} 50-\operatorname{miyj}{ }_{-50}\right) * \operatorname{inv}(\mathrm{M} 50 \mathrm{a}) *\left(y j_{-} 50-\operatorname{miyj}{ }_{-} 50\right)+$

$\operatorname{transp}\left(y j_{1} 175-\operatorname{miyj}{ }_{1} 175\right) * \operatorname{inv}(M 175 a) *\left(y j_{-} 175-\operatorname{miyj}_{-} 175\right)+$

$\operatorname{transp}\left(y j_{3} 300-\operatorname{miyj}{ }_{3} 300\right) * \operatorname{inv}(M 300 a) *\left(y j_{-300}-\operatorname{miyj} 300\right)+$

transp(yj_425 - miyj_425)* inv(M425a)* (yj_425 - miyj_425));

$\% \%$ Log da verossimilhança da iteração anterior

Lf $=(-165 / 2) * \log (2 * \mathrm{pi})-(1 / 2) *(\log (\operatorname{det}($ M25) $)+\log (\operatorname{det}($ M50) $)+$

$\log (\operatorname{det}($ M175) $)+\log (\operatorname{det}($ M300) $)+\log (\operatorname{det}($ M425) $))-(1 / 2) *$

$\left(\operatorname{transp}\left(y j_{-} 25-\operatorname{miyj} \_25\right) * \operatorname{inv}(\mathrm{M} 25) *\left(y j_{-} 25-\operatorname{miyj} 25\right)+\right.$

transp(yj_50 - miyj_50)* inv(M50)* (yj_50 - miyj_50) +

$\operatorname{transp}\left(y j \_175-\operatorname{miyj} 175\right) * \operatorname{inv}(M 175) *\left(y j \_175-\operatorname{miyj} 175\right)+$

$\operatorname{transp}\left(y j_{-300}-\operatorname{miyj} \_300\right) * \operatorname{inv}(M 300) *\left(y j_{-300}-\operatorname{miyj}\right.$ _300) +

$\operatorname{transp}\left(y j_{-4} 425-\operatorname{miyj}\right.$ 425) $_{2}$ inv(M425)*(yj_425 - miyj_425));

$\% \%$ Erro

totalerro = Lfatual $-\mathrm{Lf}$;

if abs(totalerro) < ERRO_MAX

stop $=1 ; \%$ caso positivo: flag stop

end

\% Atualização dos vetores alpha e beta que serão utilizados na

\% próxima iteração

alpha1=newalpha(1);

alpha2=newalpha (2) ;

alpha3=newalpha (3);

alpha4=newalpha (4);

alpha5=newalpha (5);

alpha6=newalpha (6);

alpha7=newalpha (7);

alpha8=newalpha (8); 


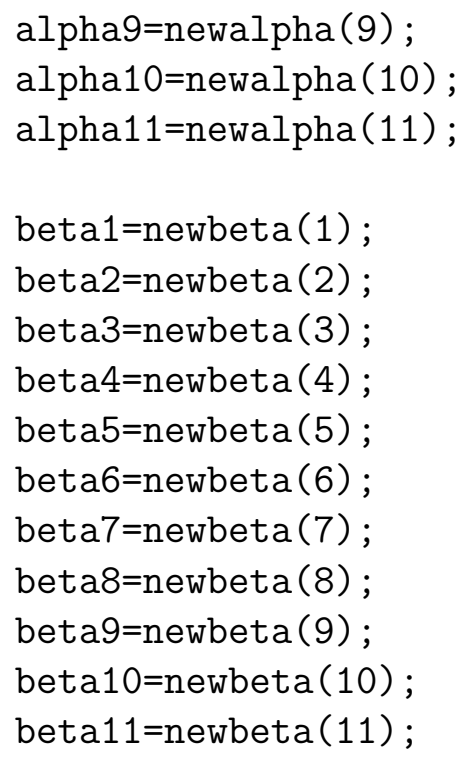

end \% Retorna ao começo do loop

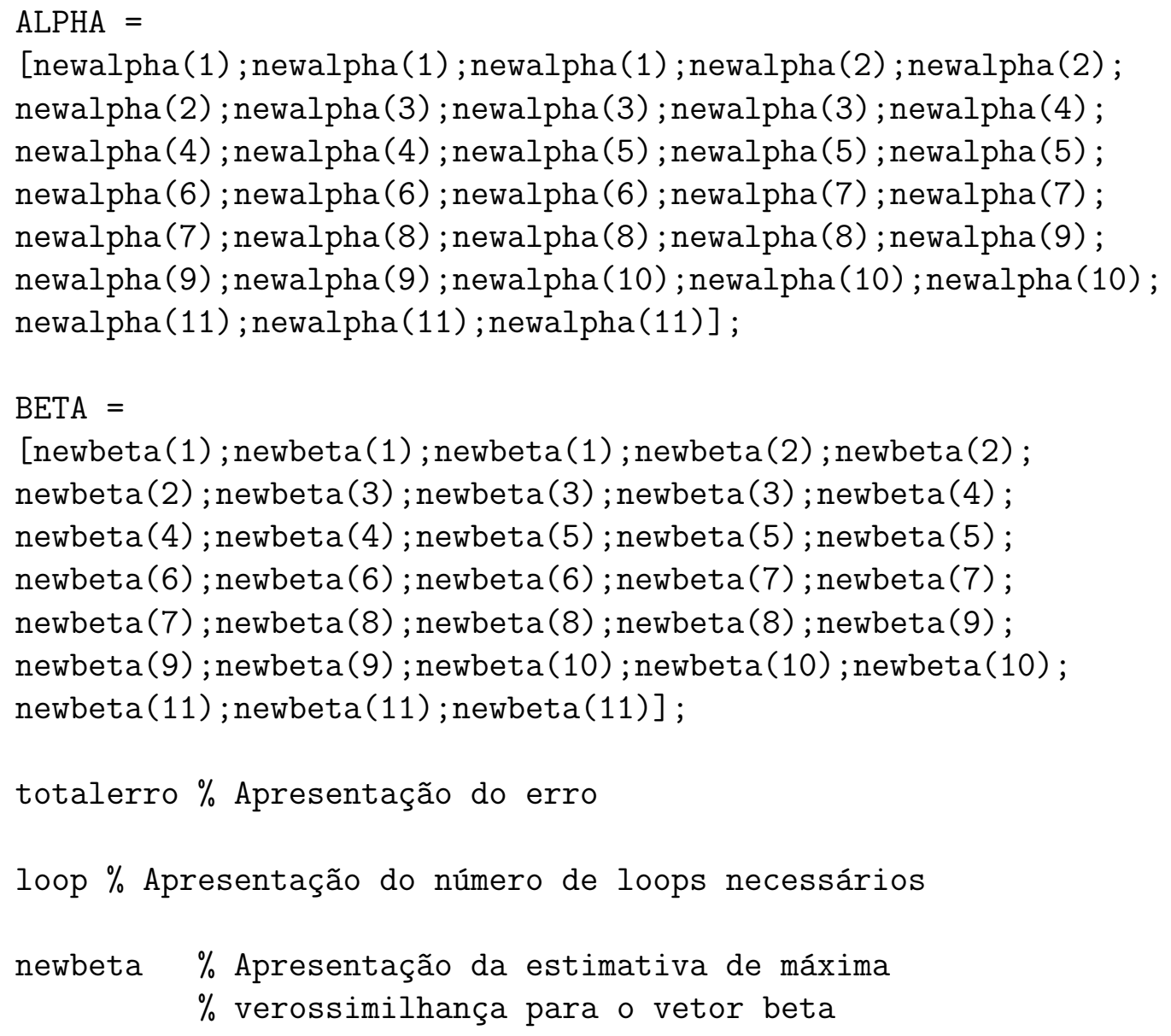

newalpha \% Apresentação da estimativa de máxima $\%$ verossimilhança para o vetor alpha

$\% \%$ Matrizes de Covariâncias obtidas a partir das estimativas de \%\% máxima verossimilhança dos parâmetros 


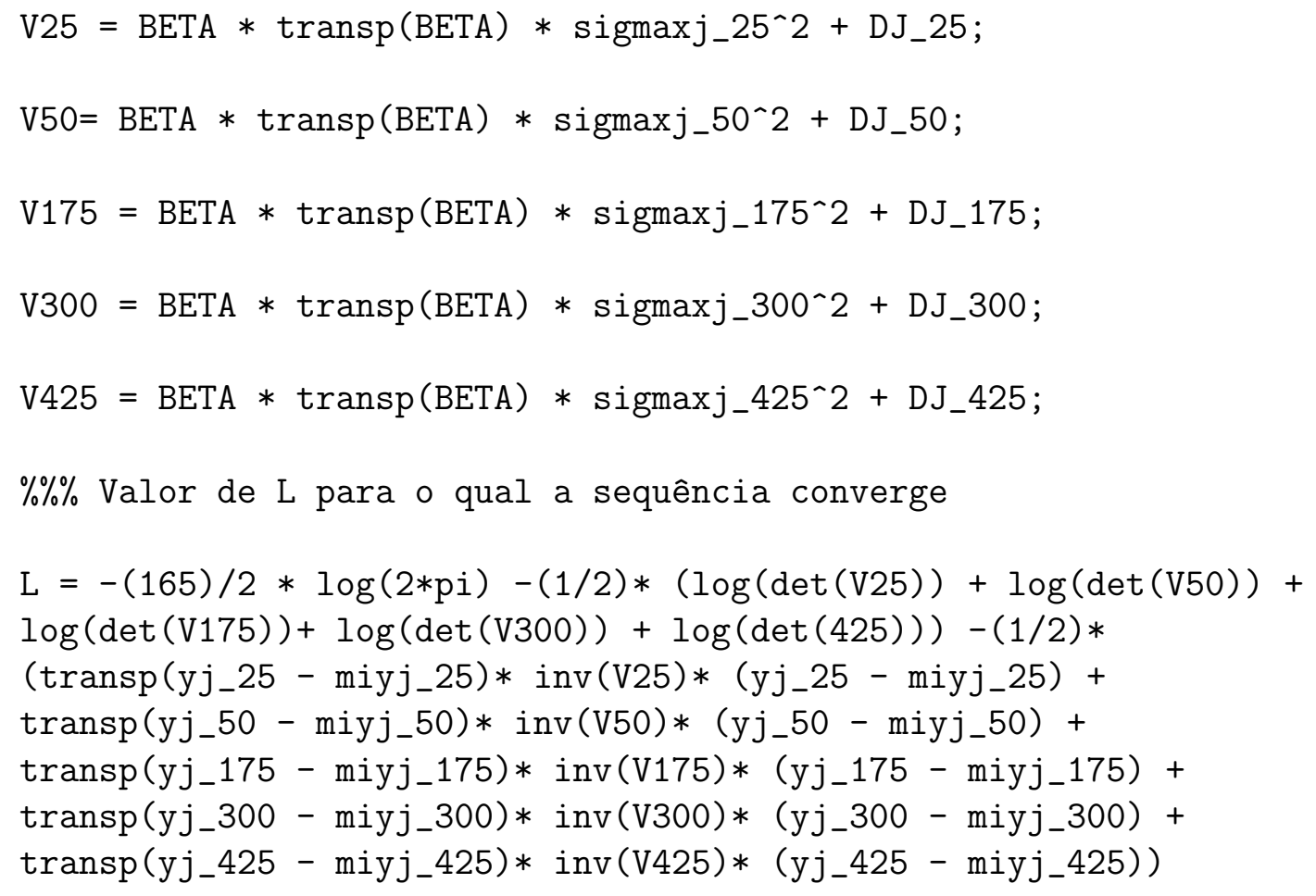




\section{Apêndice D}

\section{Função Geradora do Vetor de Médias}

\% Esta função gera o vetor miyj a partir de alpha1, alpha2, alpha3, alpha4, $\%$ alpha5, alpha6, alpha7, alpha8, alpha9, alpha10, alpha11, beta1, beta2, $\%$ beta3, beta4, beta5, beta6, beta7, beta8, beta9, beta10 e beta11, no $\%$ patamar de temperatura $j$.

$\% \%$ Arquivo feito em matlab.

function $[$ miyj] = genmiyj (alpha1, alpha2, alpha3, alpha4, alpha5, alpha6, alpha7, alpha8, alpha9, alpha10, alpha11, beta1, beta2, beta3, beta4, beta5, beta 6 , beta7, beta8, beta9, beta10, beta11, mij)

miyj=[alpha1+beta1*mij; alpha1+beta1*mij; alpha1+beta1*mij; alpha2+beta2*mij; alpha2+beta2*mij; alpha2+beta2*mij; alpha3+beta3*mij; alpha3+beta3*mij; alpha3+beta3*mij; alpha4+beta4*mij; alpha4+beta4*mij; alpha4+beta4*mij; alpha5+beta $5 *$ mij; alpha5+beta5*mij; alpha5+beta5*mij; alpha6+beta6*mij; alpha6+beta6*mij; alpha6+beta6*mij; alpha7+beta7*mij; alpha7+beta7*mij; alpha7+beta7*mij; alpha8+beta8*mij; alpha8+beta8*mij; alpha8+beta8*mij; alpha9+beta9*mij; alpha9+beta9*mij; alpha9+beta9*mij; alpha10+beta10*mij; alpha10+beta10*mij; alpha10+beta10*mij; alpha11+beta11*mij; alpha11+beta11*mij; alpha11+beta11*mij] ; 


\section{Apêndice E}

\section{Obtenção do Intervalo de Confiança}

$\% \%$ Arquivo feito em matlab

$\% \%$ Cálculo de IC para o laboratório 1

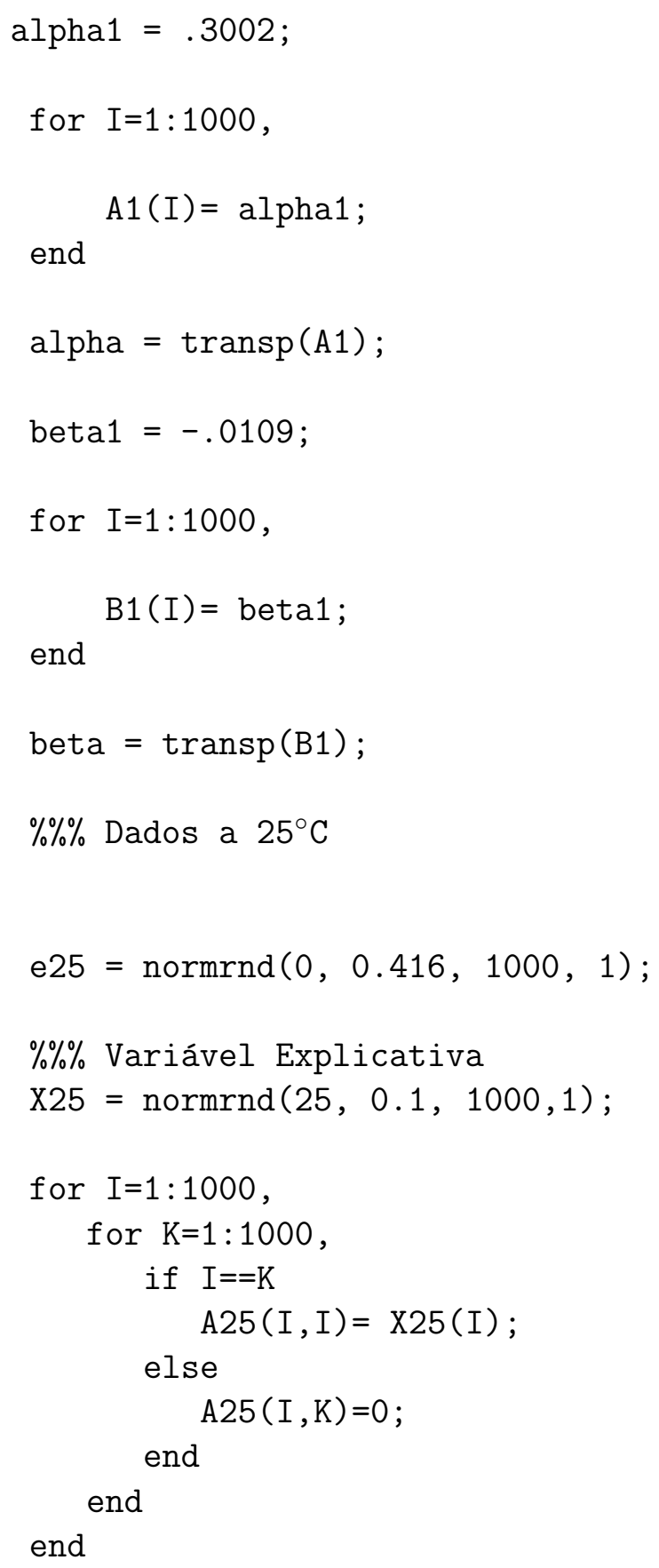




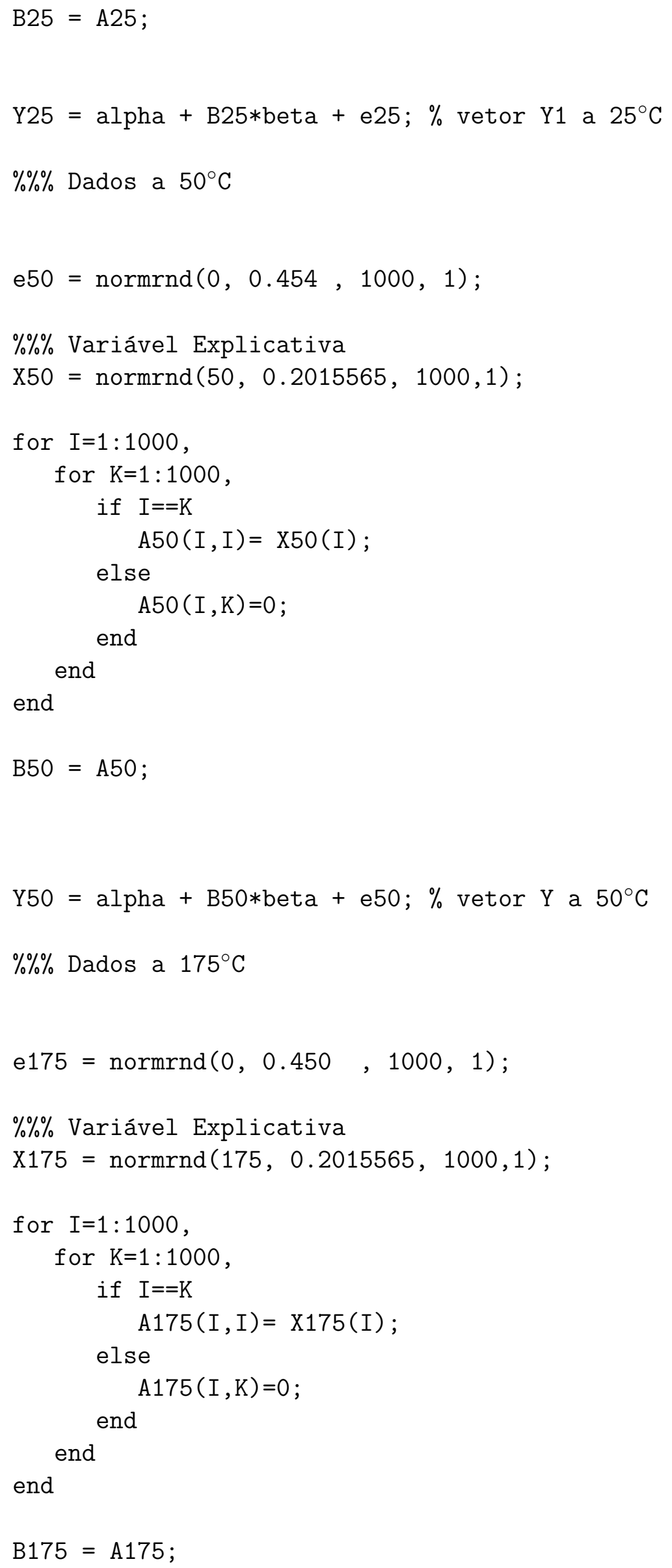




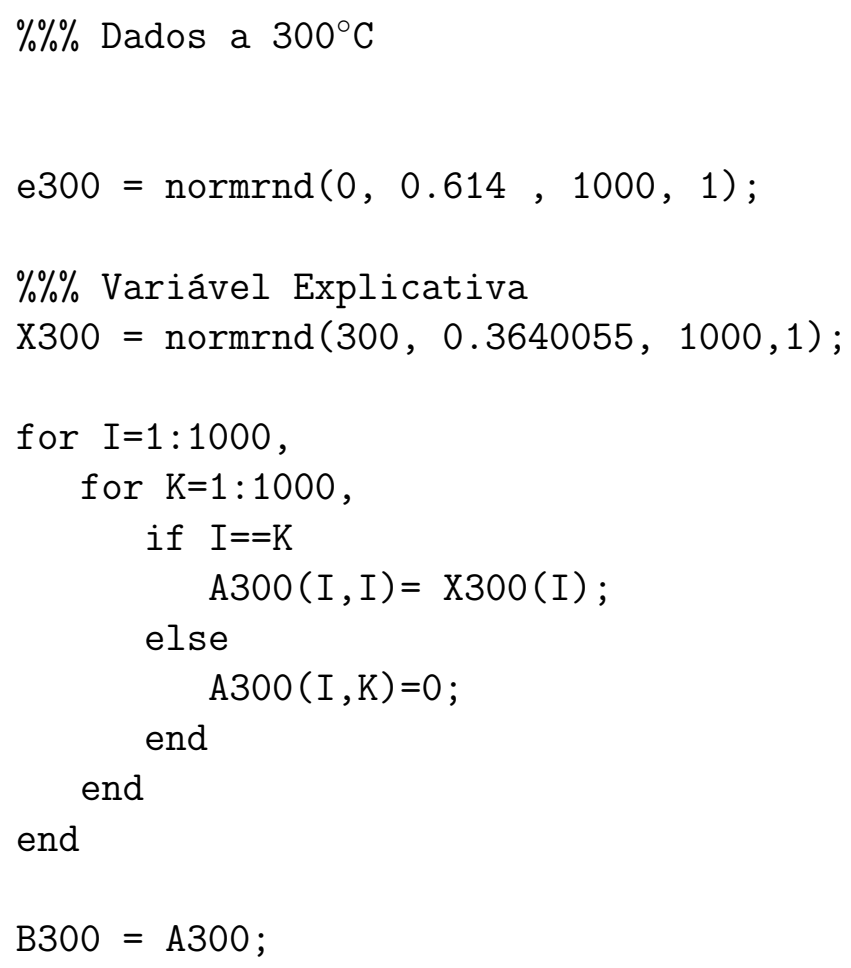




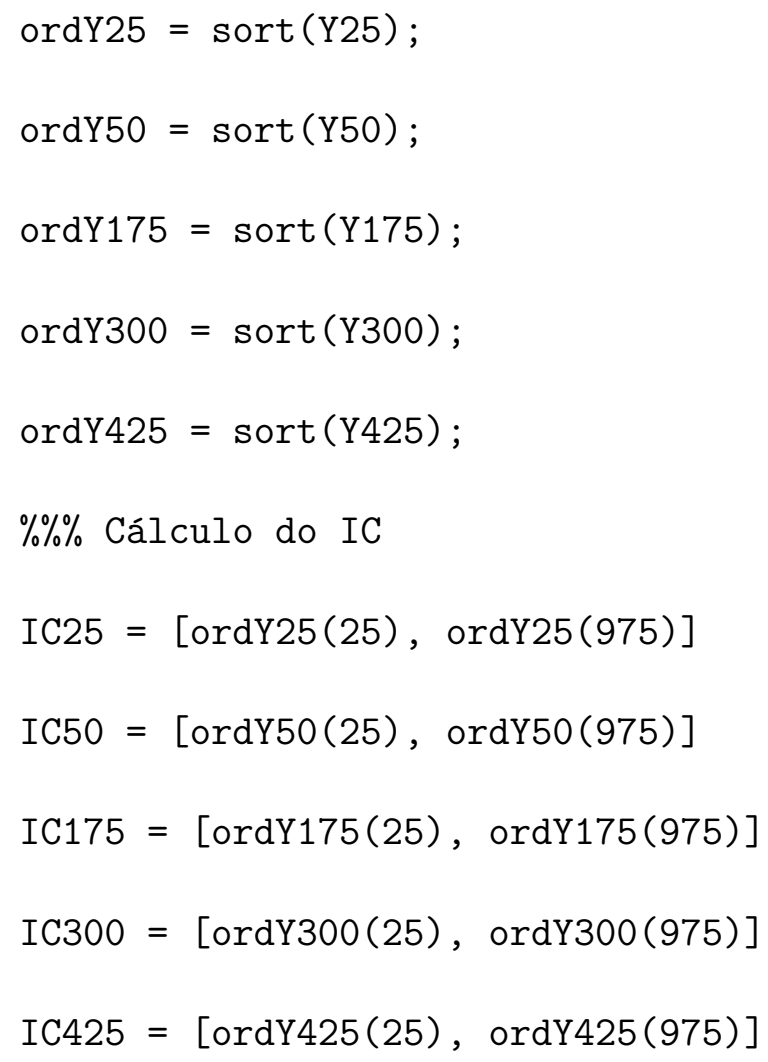




\section{Referências Bibliográficas}

[1] Boyles, R. A., On the Convergence of the EM Algorithm, Journal of the Royal Statistical Socity, B, vol 45, pg 47-50, 1983.

[2] Cheng, C. L. \& Van Ness, J. W. , Statistical Regression With Measurement Error, Kendall's Library of Statistic 6, Arnold, 1999.

[3] Dempster, A., Laird, N. \& Rubin, D. B., Maximum Likehood Estimation from incomplete data via the EM algorthm, Journal of the Royal Statistical Socity, B, vol 39, pg $1-38,1977$.

[4] Fuller, W. A., Measurement Error Models, John Willey \& Sons, 1987.

[5] Graybill, F. A., Matrices With Applications in Statistics, $2^{\text {nd }}$ edition, 1983.

[6] Grubbs, F. E. (1948) - On estimating precision of measuring instruments and product variability, Journal Amer. Stat. Assoc., 43, pg. 243-264.

[7] Grubbs, F. E. (1973) - Errors of Measurement, Precision, Accuracy and the Statistical Comparison of Measuring Instruments, Technometrics, 15, pg. 53-66.

[8] ISO/IEC Guide 43-1 (1997) - Proficiency testing by interlaboratory comparisons - Part I: Development and operation of proficiency testing schemes.

[9] ISO GUM (1998), Guia para expressão da incerteza de medição, 2 edição brasileira.

[10] Jaech, J. L. , Statistical analysis of measurement errors, Exxon Monograph, John Wiley and Sons, 1985.

[11] Lehmann, E. L., Theory of Point Estimation, John Wiley and Sons, 1983.

[12] McLachlan, G. J. \& Krishnan, T., The EM Algorithm and Extensions, John Wiley and Sons, 1997.

[13] Mandel, J., (1959) - The measuring Process, Technometrics, 1, pg. 251-267.

[14] Searle, S. R., Linear Models, John Wiley and Sons, 1971.

[15] Sen, P. K, \& Singer, J. M., (1993), Large Sample Methods in Statistics: An Introduction with Applications, Chapman \& Hall/CRC, $1^{\circ}$ ed, pg. 201 - 246.

[16] Smith, H. F. (1950) - Estimating Precision Of Measuring Instruments, Journal Amer. Stat. Assoc., 45, pg. 447-451.

[17] Srivastava, M. S. \& Khatri C. G., An Introduction to Multivariate Statistics, North Holland-New York, 1979.

[18] Thompson, W. A., Jr., (1963) - Precision Of Simultaneous Measurement Procedures, Journal Amer. Stat. Assoc., 58, pg. 474-479.

[19] Wu, C. F. J., On the Convergence Properties of the EM Algorithm, The Annals of Statistics, vol 11, No 1, pg. 95 - 103, 1983. 